\title{
Extensões conexas \\ e espaços de Banach $C(K)$ com poucos operadores
}

André Santoleri Villa Barbeiro

TESE APRESENTADA

$\mathrm{AO}$

Instituto DE Matemática E EstatísticA

DA

Universidade De SÃo Paulo

PARA

OBTENÇÃO DO TÍTULO

DE

Doutor EM CIÊNCIAS

Área de Concentração: Matemática
Orientador: Prof. Dr. Rogério Augusto dos Santos Fajardo

Durante a elaboração deste trabalho, o autor recebeu apoio financeiro da CAPES e do CNPq.

São Paulo, abril de 2018 


\section{Extensões conexas e espaços de Banach $C(K)$ com poucos operadores}

Esta versão da tese contém as correções e alterações sugeridas pela Comissão Julgadora durante a defesa da versão original do trabalho, realizada em 26/03/2018. Uma cópia da versão original está disponível no Instituto de Matemática e Estatística da Universidade de São Paulo.

Comissão Julgadora:

- Prof. Dr. Rogério Augusto dos Santos Fajardo (orientador) - IME-USP

- Prof. Dr. Antonio de Padua Franco Filho - IME-USP

- Prof. Dr. Marcelo Dias Passos - UFBA

- Prof. Dr. Pedro Levit Kaufmann - UNIFESP

- Prof. Dr. Leandro Fiorini Aurichi - ICMC-USP 
"Senhor, fazei-me instrumento de vossa paz.

Onde houver ódio, que eu leve o amor. Onde houver ofensa, que eu leve o perdão. Onde houver discórdia, que eu leve a união.

Onde houver dúvida, que eu leve a fé. Onde houver erro, que eu leve a verdade. Onde houver desespero, que eu leve a esperança. Onde houver tristeza, que eu leve a alegria. Onde houver trevas, que eu leve a luz. Ó Mestre, fazei que eu procure mais, consolar que ser consolado, compreender que ser compreendido, amar que ser amado. Pois é dando, que se recebe. É perdoando, que se é perdoado. E é morrendo que se vive para a vida eterna."

Oração de São Francisco. 


\section{Agradecimentos}

Agradeço a Deus por tudo o que Ele tem feito na minha vida.

Agradeço à minha família, por todo amor, carinho e atenção que me deram desde sempre. Ao meu pai, Alexandre, que sempre me mostrou a importância do trabalho e do esforço. À minha mãe, Áurea, que sempre me amou de modo incondicional. E à minha irmã, Adriana, que sempre esteve ao meu lado nos momentos alegres e tristes da minha vida.

Agradeço aos meus amigos Erick e Sidney, que desde os tempos de escola encontraram morada no meu coração.

Agradeço aos professores do Colégio Jardim São Paulo, que me mostraram a importância do estudo e da disciplina.

Agradeço ao meu orientador, por toda dedicação e paciência ao longo destes 4 anos.

Agradeço a todos os professores do IME que de algum modo participaram da minha formação como matemático. De maneira especial, agradeço ao professor e amigo Antonio de Padua Franco Filho.

Agradeço pelos amigos que conheci nestes 8 anos de convívio no IME. De maneira especial, Willian, Helder, Renan, Johnny, Clayton e Vini.

Agradeço ao Movimento Regnum Christi e a congregação dos Legionários de Cristo, por me mostrarem o que realmente é essencial na vida. De modo especial, agradeço aos Padres Alfonso e Sérgio pela amizade e pelo carinho. Agradeço ao ECYD por ter me trazido tantas crianças e adolescentes que me 
mostravam, a cada dia, a beleza da vida e da simplicidade.

Agradeço à CAPES e ao CNPq pelo apoio financeiro. 


\section{Resumo}

BARBEIRO, A. S. V. Extensões conexas e espaços de Banach $C(K)$ com poucos operadores. 2018. Tese de Doutorado - Instituto de Matemática e Estatística, Universidade de São Paulo, São Paulo, 2018.

Este trabalho tem dois objetivos principais. Primeiramente, analisamos a preservação de conexidade na extensão de espaços compactos por funções contínuas, técnica utilizada por Koszmider para obter $C(K)$ indecomponível com poucos operadores. Mostramos que para todo compacto metrizável $K$ existe um desconexo $L$ que é obtido a partir de $K$ por uma quantidade finita de extensões por funções contínuas. Em seguida, enfatizamos a construção de espaços de Banach da forma $C(K)$ com poucos operadores, com a propriedade de que $C(L)$ tem poucos operadores, para todo fechado $L \subseteq K$. Assumindo o princípio $\diamond$ construímos uma família $\left(K_{\xi}\right)_{\xi<2^{\left(2^{\omega}\right)}}$ de espaços conexos e hereditariamente Koszmider tais que todo operador de $C\left(K_{\xi}\right)$ em $C\left(K_{\eta}\right)$ é fracamente compacto, para $\xi \neq \eta$. Em particular, $\left(C\left(K_{\xi}\right)\right)_{\xi<2^{\left(2^{\omega}\right)}}$ é uma família de espaços de Banach indecomponíveis e dois a dois essencialmente incomparáveis, e cada espaço $K_{\xi}$ responde positivamente ao problema de Efimov. Apresentamos também um método de construção via forcing de um espaço compacto e conexo $K$ hereditariamente fracamente Koszmider.

Palavras-chave: Espaço $C(K)$, espaço indecomponível, extensão por funções contínuas, forcing, poucos operadores, espaço hereditariamente Koszmider, espaços essencialmente incomparáveis, espaço hereditariamente fracamente Koszmider, problema de Efimov, princípio diamante. 


\section{Abstract}

\section{BARBEIRO, A. S. V. Connected extensions and Banach spaces $C(K)$} with few operators. 2018. Tese de Doutorado - Instituto de Matemática e Estatística, Universidade de São Paulo, São Paulo, 2018.

This work has two main objectives. First, we analyze the preservation of connectedness in the extension of compact spaces by continuous functions, a technique used by Koszmider to obtain an indecomposable Banach space $C(K)$ with few operators. We show that for any metrizable compactum $K$ there exists a disconnected $L$ which is obtained from $K$ by finitely many extensions by continuous functions. Next, we emphasize the construction of Banach spaces of the form $C(K)$ with the property that $C(L)$ has few operators, for every closed $L \subseteq K$. Assuming the $\diamond$ principle we construct a family $\left(K_{\xi}\right)_{\xi<2^{\left(2^{\omega}\right)}}$ of connected and hereditarily Koszmider spaces such that every operator from $C\left(K_{\xi}\right)$ into $C\left(K_{\eta}\right)$ is weakly compact, for $\xi \neq \eta$. In particular, $\left(C\left(K_{\xi}\right)\right)_{\xi<2^{\left(2^{\omega}\right)}}$ is a family of indecomposable and pairwise essentially incomparable Banach spaces, and each space $K_{\xi}$ responds positively to the Efimov's problem. We also present a method of construction using forcing of a compact and connected hereditarily weakly Koszmider space $K$.

Keywords: $C(K)$ space, indecomposable space, extension by continuous functions, forcing, few operators, hereditarily Koszmider space, essentially incomparable spaces, hereditarily weakly Koszmider space, Efimov's problem, diamond principle. 


\section{Sumário}

$\begin{array}{ll}\text { Lista de Símbolos } & 11\end{array}$

$\begin{array}{ll}\text { Introdução } & 13\end{array}$

1 Definições e resultados preliminares 17

1.1 Álgebras de Boole e a Representação de Stone . . . . . . . . . 17

1.2 O espaço $C(K)$ e seu dual $M(K) \ldots \ldots . . \ldots 20$

1.3 A noção de poucos operadores em $C(K)$. . . . . . . . . . 24

1.4 O método do forcing . . . . . . . . . . . . . . . . . 27

2 Extensões por funções contínuas 35

2.1 Introdução . . . . . . . . . . . . . . . . . . . . . . 35

2.2 Definições e lemas principais . . . . . . . . . . . . . . . . 39

2.3 Supremos de álgebras de Boole e extensões por funções contínuas 42

2.4 Conexidade e desconexidade das extensões por funções contínuas 44

3 Espaços de Banach da forma $C(K)$ essencialmente incomparáveis $\quad 53$

3.1 O Axioma $\diamond \ldots \ldots \ldots \ldots \ldots \ldots$

3.2 Resultados auxiliares . . . . . . . . . . . . . . . . 58

3.3 Construção dos espaços $K_{\xi} \ldots \ldots \ldots$. . . . . . . . . . 62

4 Construção alternativa de um espaço hereditariamente fracamente Koszmider $\quad 79$

4.1 A construção do forcing . . . . . . . . . . . . . . . . . 80

4.2 Extensão Genérica . . . . . . . . . . . . . . . . . . . . . 84 
4.3 Um resultado sobre a densidade de $C(K)$ com poucos operadores 92 


\section{Lista de Símbolos}

$\mathbb{R} \quad$ o conjunto dos números reais

$\mathbb{Q} \quad$ o conjunto dos números racionais

$\omega \quad$ o conjunto dos naturais e o primeiro ordinal infinito enumerável

$\omega_{1} \quad$ o menor cardinal não enumerável

$\omega_{2} \quad$ o segundo cardinal não enumerável

$2^{\omega} \quad$ o cardinal do contínuo

$\diamond \quad$ o princípio diamante

$\mathrm{CH}$ a hipótese do contínuo, isto é, $\omega_{1}=2^{\omega}$

GCH a hipótese generalizada do contínuo, isto é, $\omega_{\alpha+1}=2^{\omega_{\alpha}}$ para todo ordinal $\alpha$

$C(K)$ o espaço de Banach das funções contínuas de $K$ em $\mathbb{R}$ com a norma supremo

$M(K)$ o espaço de Banach das medidas de Radon sobre $K$ com a norma da variação

$\|x\| \quad$ a norma de $x$

$X^{*} \quad$ o espaço dual do espaço de Banach $X$

$T^{*} \quad$ o operador adjunto do operador $T$

$l_{\infty} \quad$ o espaço de Banach das sequências limitadas em $\mathbb{R}$ com a norma supremo

$c_{0} \quad$ o espaço de Banach das sequências reais convergentes a 0, com a norma supremo

$X \oplus Y \quad$ a soma direta de $X$ e $Y$

$\beta \mathbb{N} \quad$ o compactificado de Stone-Čech dos naturais

$\operatorname{Ker}(f)$ o núcleo da função $f$

$\operatorname{Im}(f)$ a imagem da função $f$

$\operatorname{dom}(f)$ o domínio da função $f$

$\operatorname{supp}(f)$ o suporte da função $f$, isto é, $\overline{\{x \in d o m(f): f(x) \neq 0\}}$

$G r(f) \quad$ o gráfico da função $f$, isto é, $\{(x, f(x)): x \in \operatorname{dom}(f)\}$

ZFC o sistema de axiomas de Zermelo-Frankel com Axioma da Escolha

$p \Vdash \quad$ a condição $p$ força que 


\section{Introdução}

Um espaço de Banach de dimensão infinita X é dito decomponível se, e somente se, existem subespaços fechados Y e Z, ambos de dimensão infinita, tais que $X=Y \oplus Z$. Se $\mathrm{X}$ não for decomponível, diremos que $\mathrm{X}$ é indecomponível. Durante muito tempo, a seguinte questão concernente à teoria dos espaços de Banach ficou em aberto: será que todo espaço de Banach de dimensão infinita é decomponível?

Em 1993, Gowers e Maurey responderam negativamente a esta pergunta, construindo, em [GM1], o primeiro exemplo de um espaço de Banach indecomponível $X_{G M}$. Mais do que apenas indecomponível, o espaço $X_{G M}$ é hereditariamente indecomponivel, i.e., todos os seus subespaços fechados de dimensão infinita são indecomponíveis.

No exemplo construído por Gowers e Maurey, todo operador linear e contínuo em $X_{G M}$ pode ser escrito da forma $\gamma I+S$, onde $\gamma$ é um número real, $I: X_{G M} \longrightarrow X_{G M}$ é o operador identidade e $S: X_{G M} \longrightarrow X_{G M}$ é um operador estritamente singular (i.e., $S$ é um operador linear e contínuo tal que, para todo subespaço de dimensão infinita $Z \subseteq X_{G M}$, a restrição $\left.f\right|_{Z}: Z \longrightarrow X_{G M}$ não é um isomorfismo sobre a imagem).

O primeiro exemplo de um espaço de Banach indecomponível da forma $C(K)$ foi construído por Piotr Koszmider em [Ko2]. Em [Ko2], Koszmider mostra que, se $K$ é um compacto Hausdorff tal que todo operador linear e contínuo em $C(K)$ é multiplicador fraco, então $C(K)$ não é isomorfo a qualquer um dos seus subespaços próprios e nem a qualquer um dos seus quocientes próprios. Em particular, $C(K)$ não é isomorfo a qualquer um dos seus hiperplanos. Além disso, se $K \backslash F$ é conexo para todo subconjunto finito $F \subseteq K$, então $C(K)$ é indecomponível. 
Ainda em [Ko2], assumindo a Hipótese do Contínuo (CH) Koszmider constrói o primeiro exemplo de um espaço de Banach $C(K)$ indecomponível em que todo operador linear e contínuo é multiplicação fraca. Utilizando a Representação de Wallman (uma generalização, para reticulados, do Teorema da Representação de Stone) Plebanek, em [Pl], constrói dentro de ZFC um espaço de Banach $C(K)$ indecomponível em que todo operador linear e contínuo é multiplicação fraca.

Como todo espaço de Banach da forma $C(K)$ de dimensão infinita possui uma cópia de $c_{0}$ como subespaço segue que, diferentemente do espaço $X_{G M}$, $C(K)$ não pode ser hereditariamente indecomponível. O espaço construído em [Ko2] é, portanto, um exemplo natural de espaço de Banach indecomponível que não é hereditariamente indecomponível. Em [Fer], Ferenczi mostra que o espaço $X_{G M}$, além de hereditariamente indecomponível, é quociente hereditariamente indecomponível, isto é, todos os seus quocientes são hereditariamente indecomponíveis. Por um resultado de [LM], temos que espaços de Banach da forma $C(K)$ possuem $c_{0}$ complementado ou $l_{2}$ como quociente e, portanto, um espaço da forma $C(K)$ também não pode ser quociente hereditariamente indecomponível.

Em [Pe2], Pełczyński mostra que um operador $T: C(K) \longrightarrow C(K)$ é fracamente compacto se, e somente se, é estritamente singular. Em [Ko2], Koszmider prova que, diferentemente do espaço $X_{G M}$ construído por Gowers e Maurey, não existem espaços de Banach da forma $C(K)$ em que todo operador linear e contínuo possa ser escrito como $\gamma I+S$, onde $\gamma \in \mathbb{R}$, $I: C(K) \longrightarrow C(K)$ é o operador identidade e $S: C(K) \longrightarrow C(K)$ é um operador estritamente singular (equivalentemente, fracamente compacto). Também em [Ko2], mostra-se que todo espaço de Banach $C(K)$ possui um operador linear e contínuo que não é da forma $g I+C$, onde $g \in C(K)$ e $C: C(K) \longrightarrow C(K)$ é um operador compacto. Logo, no contexto dos espaços de Banach da forma $C(K)$, o máximo que podemos esperar sobre poucos operadores é que todo operador linear e contínuo em $C(K)$ seja multiplicação fraca.

Assumindo diamante $(\diamond)$, um princípio combinatório mais forte que $\mathrm{CH}$, Rogério Fajardo, em [Fa2], constrói um compacto Hausdorff e conexo $K$ tal 
que, para todo fechado $L \subseteq K, C(L)$ possui poucos operadores, no sentido de que todo operador linear e contínuo em $\mathrm{C}(\mathrm{L})$ é multiplicação fraca. Em particular, o espaço $K$ construído por Fajardo responde positivamente ao problema de Efimov, sobre a existência de um espaço compacto que não contém cópia de $\beta \mathbb{N}$ e nem sequências convergentes não triviais. Utilizando a técnica do forcing, Fajardo mostra, em [Fa1], que é consistente com ZFC que existe um espaço de Banach da forma $C(K)$ indecomponível de densidade $\omega_{1}<2^{\omega}$ tal que todo operador linear e contínuo em $C(K)$ é multiplicação fraca. Também utilizando a técnica do forcing, Koszmider, em [Ko5], mostra que é consistente com ZFC que existe um espaço de Banach $C(K)$ indecomponível de densidade $2^{2^{\omega}}=\omega_{2}>\omega_{1}=2^{\omega}$ tal que todo operador linear e contínuo em $C(K)$ é multiplicação fraca. Recentemente, Koszmider, Shelah e Świetek mostraram, em [Ko6], que não existe limitante superior para a densidade de um espaço de Banach indecomponível. Os autores provam, assumindo a Hipótese Generalizada do Continuum (GCH), que para todo cardinal $\kappa$ existe um espaço de Banach indecomponível da forma $C(K)$ com poucos operadores de densidade maior que $\kappa$. Tal espaço não possui subespaço complementado de dimensão infinita com densidade menor que $\kappa$, o que também fornece um resultado mais forte que o obtido em [Ko3].

Dizemos que dois espaços de Banach são totalmente incomparáveis se nenhum subespaço de dimensão infinita de um espaço for isomorfo a um subespaço do outro. Sabemos que $C(K)$ de dimensão infinita possui uma cópia de $c_{0}$ como subespaço e, portanto, espaços de Banach da forma $C(K)$ não podem ser totalmente incomparáveis. Dois espaços de Banach $X$ e $Y$ são ditos essencialmente incomparáveis se para todos os operadores $T: X \longrightarrow Y$ e $S: Y \longrightarrow X$ o operador $I-S \circ T: X \longrightarrow X$ é um operador de Fredholm. Fajardo, em [Fa3], constrói em ZFC uma família de tamanho $2^{\omega}$ de espaços de Banach indecomponíveis da forma $C(K)$ com poucos operadores que são dois a dois essencialmente incomparáveis. Além disso, assumindo o princípio diamante $(\diamond)$, Fajardo obtém uma tal família de tamanho $2^{2^{\omega}}$.

Para obter $C(K)$ indecomponível com poucos operadores, Koszmider, em [Ko2], utiliza a técnica de extensões por funções contínuas. A condição de ser uma extensão forte foi utilizada para garantir a conexidade da extensão, 
conforme mostrado em [Ko2]. No capítulo 2 da presente tese, abordamos a seguinte pergunta: para preservarmos a conexidade, é realmente necessário exigirmos que a extensão seja forte? Será que uma extensão sempre preserva conexidade, seja ela forte ou não? Provamos que extensões de compactos métricos não necessariamente preservam conexidade. Com efeito, obtivemos os três resultados a seguir:

i) Existe uma extensão desconexa de um compacto conexo e metrizável $K$

ii) Para todo compacto metrizável $K$ existe um desconexo $L$ que é obtido a partir de $K$ por uma quantidade finita de extensões por funções contínuas;

iii) Se $K$ é compacto conexo, localmente conexo e metrizável, então toda extensão de $K$ por funções contínuas é conexa (mas pode não ser localmente conexa).

Também no capítulo 2, mostramos a relação que existe entre adicionar supremos em álgebras de Boole e a técnica de extensões por funções contínuas.

No capítulo 3, unindo as técnicas presentes em [Fa2] e [Fa3] construímos, assumindo $\diamond$, uma família $\left(K_{\xi}\right)_{\left.\xi<2^{(2 \omega}\right)}$ de espaços conexos e hereditariamente Koszmider tais que $C\left(K_{\xi}\right)$ e $C\left(K_{\eta}\right)$ são essencialmente incomparáveis, para todo $\xi \neq \eta$. Como espaços hereditariamente Koszmider não possuem cópia de $\beta \mathbb{N}$ nem sequências convergentes não trivias, cada $K_{\xi}$ construído responde positivamente ao problema de Efimov. No capítulo 4, apresentamos um método alternativo de construção via forcing de espaços $C(K)$ com poucos operadores com a propriedade de que $C(L)$ também tem poucos operadores, para todo fechado $L \subseteq K$. Em particular, o compacto $K$ obtido via forcing também responde positivamente ao problema de Efimov. No final do capítulo 4 apresentamos um resultado envolvendo a densidade dos espaços de Banach $C(K)$ com poucos operadores. Por fim, no capítulo 1 tratamos de algumas definições e de alguns resultados preliminares que serão utilizados ao longo de toda a tese.

Todos os espaços considerados ao longo da tese são Hausdorff. 


\section{Capítulo 1}

\section{Definições e resultados preliminares}

O objetivo principal deste capítulo é apresentar algumas definições e alguns resultados que serão utilizados ao longo de toda a tese. A Seção 1.1 relembra as definições de reticulado e álgebras de Boole, bem como o importante teorema da representação de Stone, que associa biunivocamente álgebras de Boole com espaços booleanos. A seção 1.2 apresenta as medidas de Radon e o espaço $M(K)$. A seção 1.3 apresenta definições e resultados que aparecem em [Ko2], [Ko4], [Ko5] e [Ko6]. Por fim, a seção 1.4 apresenta as noções básicas e alguns resultados fundamentais envolvendo o método do forcing.

\section{1 Álgebras de Boole e a Representação de Stone}

Definição 1.1. Um conjunto $R$, parcialmente ordenado por $\leq$, é um reticulado se, para todo $x, y \in R$, existirem $\inf \{x, y\}$ (indicado por $x \wedge y$ ) e $\sup \{x, y\}$ (indicado por $x \vee y$ ). Diremos que o reticulado é completo se todo subconjunto de $R$ tem supremo e ínfimo.

Definição 1.2. Um reticulado $R$ é distributivo se, para todo $x, y, z \in R$, 
valerem as igualdades

$$
x \wedge(y \vee z)=(x \wedge y) \vee(x \wedge z)
$$

e

$$
x \vee(y \wedge z)=(x \vee y) \wedge(x \vee z)
$$

Definição 1.3. Um reticulado $R$ é complementado se satisfizer as duas condições a seguir:

(a) Existem dois elementos distintos $0 \in R$ e $1 \in R$ tais que, para todo $x \in R$, temos $0 \leq x \leq 1$;

(b) Para todo $x \in R$ existe $-x \in R$, chamado o complemento de $x$, tal que $x \vee(-x)=1$ e $x \wedge(-x)=0$.

Definição 1.4. Uma álgebra de Boole é um reticulado distributivo e complementado. Diremos que a álgebra de Boole é completa se o reticulado associado à ela for completo.

Definição 1.5. Seja $M$ um subconjunto de uma álgebra de Boole $A$. Então

$$
\langle M\rangle=\bigcap\{B \subseteq A: B \text { é subálgebra de } A \text { e } M \subseteq B\}
$$

é a subálgebra gerada por $M$.

Definição 1.6. Dadas duas álgebras de Boole $A$ e $B$, um homomorfismo entre $A$ e $B$ é uma função $h: A \longrightarrow B$ satisfazendo

$$
h(0)=0, h(1)=1
$$

e, para todo $x, y \in A$,

$$
h(x \wedge y)=h(x) \wedge h(y), h(x \vee y)=h(x) \vee h(y), h(-x)=-h(x)
$$

Um isomorfismo é um homomorfismo bijetor. Denotamos por $A \cong B$ quando $A$ é isomorfo a $B$. 
Dada uma álgebra de Boole $A$, existe álgebra de Boole completa $B$ tal que $A$ é subálgebra densa de $B$. A álgebra $B$ é única a menos de isomorfismo (veja [Kop]) e se chama o completamento de A.

Definição 1.7. Seja $A$ uma álgebra de Boole. Dizemos que $F \subseteq A$ é um filtro em $A$ se

- $1 \in F$;

- $0 \notin F$;

- Se $x, y \in F$ então $x \wedge y \in F$;

- Para todo $x, y \in A$, se $x \in F$ e $x \leq y$, então $y \in F$.

Um ultrafiltro em A é um filtro maximal, isto é, um filtro que não está contido propriamente em nenhum outro filtro.

Definição 1.8. Seja $A$ uma álgebra de Boole. Dizemos que $F \subseteq A$ tem a propriedade da intersecção finita (p.i.f.) se para todos $x_{1}, \ldots, x_{n} \in F$ temos $x_{1} \wedge \ldots \wedge x_{n} \neq 0$

Lema 1.9. Seja A uma álgebra de Boole.

(a) Se $F \subseteq A$ tem a propriedade da intersecção finita então existe um ultrafiltro que contém $F$;

(b) Um filtro $F$ em $A$ é um ultrafiltro se e somente se para todo a $\in A$, ou $a \in F$ ou $-a \in F$;

(c) Dados $a, b \in A$ distintos, existe um ultrafiltro $\mathcal{U}$ em $A$ tal que $a \in \mathcal{U} e$ $b \notin \mathcal{U}$, ou $b \in \mathcal{U}$ e $a \notin \mathcal{U}$.

Definição 1.10. Seja $X$ um espaço topológico. Diremos que $X$ é localmente conexo se cada $x \in X$ admite uma base de vizinhanças abertas e conexas.

Definição 1.11. Seja $X$ um espaço topológico. Diremos que $X$ é 0 -dimensional se $X$ possuir uma base topológica formada por subconjuntos abertos-fechados de $X$. 
Definição 1.12. Seja $X$ um espaço topológico. Diremos que $X$ é totalmente desconexo se os únicos subconjuntos conexos não-vazios de $X$ são os unitários.

Teorema 1.13. ([Wa], 2.4) Um espaço topológico compacto é 0-dimensional se, e somente se, é totalmente desconexo.

Definição 1.14. O peso de um espaço topológico $X, \omega(X)$, é o menor cardinal infinito $\kappa$ tal que existe uma base topológica de $X$ de cardinalidade menor ou igual a $\kappa$.

Definição 1.15. Dado $K$ um espaço topológico compacto 0-dimensional, definimos $C l o p(K)$ a álgebra de Boole dos abertos-fechados de $K$. Dada uma álgebra de Boole $A$, definimos o espaço de Stone de $A$, denotado $S(A)$, como o conjunto dos ultrafiltros em $A$ munido da topologia gerada por $\left\{a^{*}: a \in A\right\}$, onde $a^{*}=\{u \in S(A): a \in u\}$.

Observação. Também usaremos a notação $[a]$ para indicar o conjunto de todos os ultrafiltros que contêm $a$.

Teorema 1.16 (Stone). Seja A uma álgebra de Boole. Então $A \cong C \operatorname{lop}(S(A))$ pelo isomorfismo $h: A \longrightarrow C l o p(S(A))$ dado por $h(a)=a^{*}$. Além disso, para todo espaço topológico compacto 0-dimensional $K$, temos $K$ homeomorfo a $S(C l o p(K))$.

Da Definição 1.15 segue imediatamente o seguinte Lema:

Lema 1.17. Se A é uma álgebra de Boole então $\omega(S(A))=|A|$.

\section{$1.2 \quad$ O espaço $C(K)$ e seu dual $M(K)$}

Os borelianos sobre um espaço topológico $X$ são os elementos da $\sigma$-álgebra gerada pelos abertos de $X$; isto é, o menor subconjunto de $\mathcal{P}(X)$ que contém os abertos de $X$ e é fechado por uniões enumeráveis e complementos.

Notação 1.18. Dado um espaço topológico $X$, denotamos por $\operatorname{Bor}(X)$ a $\sigma$-álgebra dos borelianos de $X$. 
Definição 1.19. Seja $K$ um espaço topológico compacto. Uma medida boreliana $\mu$ sobre $K$ é uma função $\mu: \operatorname{Bor}(K) \longrightarrow \mathbb{R}$ que satisfaz as seguintes condições:

- $\mu(\emptyset)=0$;

- Para toda família $\left(B_{n}\right)_{n \in \omega}$ de borelianos de $K$ dois a dois disjuntos, tem-se que

$$
\mu\left(\bigcup_{n \in \omega} B_{n}\right)=\Sigma_{n \in \omega} \mu\left(B_{n}\right)
$$

Definição 1.20. Seja $K$ um espaço topológico compacto. Uma medida boreliana $\mu$ sobre $K$ é dita positiva se $\mu(B) \geq 0$, para todo $B \in \operatorname{Bor}(K)$.

Definição 1.21. Dados um espaço topológico compacto $K$ e uma medida boreliana $\mu$ sobre $K$, definimos $|\mu|$ por

$|\mu|(B)=\sup \left\{\Sigma_{1 \leq i \leq n}\left|\mu\left(B_{i}\right)\right|: B_{i} \in \operatorname{Bor}(K), B_{i} \subseteq B, B_{i} \cap B_{j}=\emptyset\right.$, se $\left.i \neq j\right\}$,

onde $B \in \operatorname{Bor}(K)$.

Temos que $|\mu|$ está bem definida como função de $\operatorname{Bor}(K)$ em $\mathbb{R}$ e é uma medida positiva (veja $[\mathrm{Ru}]$ ). Chamamos a medida $|\mu|$ de variação de $\mu$.

Definição 1.22. Sejam $K$ um espaço topológico compacto e $\mu$ uma medida boreliana sobre $K$. Dizemos que $\mu$ é regular se para todo $B \in \operatorname{Bor}(K)$ e todo $\varepsilon>0$ existem $F$ fechado e $G$ aberto tais que $F \subseteq B \subseteq G$ e $|\mu|(G \backslash F)<\varepsilon$.

Uma medida de Radon sobre um espaço topológico compacto $K$ é simplesmente uma medida boreliana regular sobre $K$. De agora em diante, convencionaremos que o termo medida será usado no sentido de medida de Radon. Segue imediatamente da definição de regularidade que, se $\mu$ é regular, então $|\mu|$ também é regular.

O próximo lema nos será bastante útil ao longo da tese. Ele nos diz que uma medida sobre um espaço compacto $K$ está unicamente determinada por seus valores em uma base de $K$ fechada por intersecções finitas. 
Lema 1.23. Sejam $K$ um espaço topológico compacto e $B$ uma base de $K$ fechada por intersecções finitas. Se $\mu$ e $\nu$ são medidas sobre $K$ satisfazendo $\left.\mu\right|_{B}=\left.\nu\right|_{B}$, então $\mu=\nu$.

Demonstração. Se $E_{1}$ e $E_{2}$ são borelianos em $K$, satisfazendo $E_{1} \subseteq E_{2}$, a $\sigma$-aditividade de $\mu$ e $\nu$ nos dizem que $\mu\left(E_{2} \backslash E_{1}\right)=\mu\left(E_{2}\right)-\mu\left(E_{1}\right)$ e também que $\nu\left(E_{2} \backslash E_{1}\right)=\nu\left(E_{2}\right)-\nu\left(E_{1}\right)$. Provaremos agora que, se $V$ é uma união finita de elementos de $B$, então $\mu(V)=\nu(V)$. Para isso, mostraremos por indução em $n$ que, se $X \subseteq B$ e $|X|=n$, então $\mu(\cup X)=\nu(\cup X)$. Com efeito, o passo inicial $n=1$ é garantido pela hipótese do Lema. Suponha agora que $\mu$ e $\nu$ coincidem para todas as uniões de no máximo $n$ elementos de $B$ e defina $V=\cup\left\{V_{i}: 1 \leq i \leq n+1\right\}$, onde $V_{i} \in B$. Note que

$$
V=\left(V_{n+1} \backslash \bigcup_{1 \leq i \leq n}\left(V_{n+1} \cap V_{i}\right)\right) \cup\left(\bigcup_{1 \leq i \leq n} V_{i}\right)
$$

Como $B$ é fechado por intersecções finitas, temos $V_{n+1} \cap V_{i} \in B$. Sejam agora $V^{\prime}=\bigcup_{1 \leq i \leq n}\left(V_{n+1} \cap V_{i}\right)$ e $V^{\prime \prime}=\bigcup_{1 \leq i \leq n}\left(V_{i}\right)$. Usando $(*)$, a hipótese indutiva e o fato de que $\mu$ e $\nu$ concidem em $B$, segue que

$$
\mu(V)=\mu\left(V_{n+1}\right)-\mu\left(V^{\prime}\right)+\mu\left(V^{\prime \prime}\right)=\nu\left(V_{n+1}\right)-\nu\left(V^{\prime}\right)+\nu\left(V^{\prime \prime}\right)=\nu(V) .
$$

Considere $E \in \operatorname{Bor}(K)$ qualquer. Usando a regularidade de $\mu$ e $\nu$ obtemos, para cada natural $n>0$, fechados $F_{n}^{1}$ e $F_{n}^{2}$ contidos em $E$ e abertos $G_{n}^{1}$ e $G_{n}^{2}$ contendo $E$ tais que $|\mu|\left(G_{n}^{1} \backslash F_{n}^{1}\right)<\frac{1}{4 n}$ e $|\nu|\left(G_{n}^{2} \backslash F_{n}^{2}\right)<\frac{1}{4 n}$. Definindo $G_{n}=G_{n}^{1} \cap G_{n}^{2}$ e $F_{n}=F_{n}^{1} \cup F_{n}^{2}$, temos $F_{n} \subseteq E \subseteq G_{n},|\mu|\left(G_{n} \backslash F_{n}\right)<\frac{1}{4 n}$ e $|\nu|\left(G_{n} \backslash F_{n}\right)<\frac{1}{4 n}$. Observe que $F_{n}$ é compacto pois $K$ é compacto e $F_{n}$ é um fechado de $K$. Logo, existe $W_{n}$ união finita de abertos pertencentes a $B$ tal que $F_{n} \subseteq W_{n} \subseteq G_{n}$. Dessa forma, temos

$$
\begin{gathered}
\left|\mu(E)-\mu\left(W_{n}\right)\right| \leq\left|\mu(E)-\mu\left(F_{n}\right)\right|+\left|\mu\left(W_{n}\right)-\mu\left(F_{n}\right)\right|= \\
\left|\mu\left(E \backslash F_{n}\right)\right|+\left|\mu\left(W_{n} \backslash F_{n}\right)\right| \leq|\mu|\left(E \backslash F_{n}\right)+|\mu|\left(W_{n} \backslash F_{n}\right)<\frac{1}{2 n} .
\end{gathered}
$$

Analogamente, segue também que $\left|\nu(E)-\nu\left(W_{n}\right)\right|<\frac{1}{2 n}$. Logo, como vale 
$\mu\left(W_{n}\right)=\nu\left(W_{n}\right)$, concluímos que

$$
|\mu(E)-\nu(E)| \leq\left|\mu(E)-\mu\left(W_{n}\right)\right|+\left|\nu(E)-\nu\left(W_{n}\right)\right|<\frac{1}{n},
$$

para todo $n>0$. Fazendo $n \rightarrow \infty$, segue que $\mu(E)=\nu(E)$.

Definição 1.24. Sejam $I$ um conjunto e $\left\{X_{i}: i \in I\right\}$ uma família de espaços topológicos. O produto de Tychonoff dessa família é o espaço topológico $X=\prod_{i \in I} X_{i}$ cuja topologia é a gerada pela base

$\left\{\Pi_{i \in I} U_{i}: U_{i}\right.$ é aberto em $X_{i}$, para todo $i \in I$, e $\left\{i \in I: U_{i} \neq X_{i}\right\}$ é finito $\}$.

Definição 1.25. Seja $\Pi_{\alpha<\kappa} X_{\alpha}$ um produto de espaços topológicos, para $\kappa$ um ordinal limite. Sejam $Y_{\alpha}$ subespaços de $\Pi_{\beta<\alpha} X_{\beta}$ tais que $\pi_{\beta}\left[Y_{\alpha}\right]=Y_{\beta}$, para $\beta<\alpha$. Definimos o limite inverso de $\left(Y_{\alpha}\right)_{\alpha<\kappa}$ por

$$
\lim _{\leftarrow}\left(Y_{\alpha}\right)_{\alpha<\kappa}=\left\{\left(y_{\alpha}\right)_{\alpha<\kappa} \in \Pi_{\alpha<\kappa} X_{\alpha}: \forall \alpha<\kappa\left(\left(y_{\beta}\right)_{\beta<\alpha} \in Y_{\alpha}\right)\right\}
$$

Limite inverso preserva compacidade (veja [Eng], 2.5.1).

Definimos um aberto elementar de $\prod_{i \in I} X_{i}$ como sendo um aberto da forma $\prod_{i \in I} U_{i}$, onde cada $U_{i}$ é um aberto básico (para uma base fixada) de $X_{i}$ e $U_{i}=X_{i}$ para todos, exceto finitos, $i \in I$. Além disso, se $Y \subseteq \Pi_{i \in I} X_{i}$ é um subespaço, chamamos de aberto elementar de $Y$ um aberto elementar de $\prod_{i \in I} X_{i}$ intersectado com $Y$. Observe que, da definição de topologia produto e da definição de subespaço, segue que os abertos elementares formam uma base de abertos para $Y$, fechada por intersecções finitas. No caso em que tivermos $X_{i}=[0,1]$, consideraremos como abertos básicos os intervalos abertos de $[0,1]$ com extremos racionais.

Definição 1.26. Dado um cardinal $\alpha$, denotaremos por $\mathcal{B}_{\alpha}$ a base de abertos elementares de extremos racionais do espaço $[0,1]^{\alpha}$.

Observe que pelo Lema 1.23 podemos identificar medidas de Radon sobre $[0,1]^{\alpha}$ com funções de $\mathcal{B}_{\alpha}$ em $\mathbb{R}$. 
Notação 1.27. Seja $K$ um espaço topológico compacto. Denotamos por $M(K)$ o conjunto das medidas de Radon sobre $K$ e, para cada $\mu \in M(K)$, denotamos por $\|\mu\|=|\mu|(K)$.

O Teorema da Representação de Riesz nos permite identificar o espaço dual $C(K)^{*}$ de $C(K)$ com o espaço $M(K)$ das medidas de Radon sobre $K$, munido da norma $\|\mu\|=|\mu|(K)$. Faremos essa identificação ao longo de toda a tese e consideraremos sempre as topologias fraca e fraca* de $M(K)$. Sugerimos [Fa], [Se] ou [Di] para referências.

O próximo Teorema, também conhecido como o Lema de Rosenthal, será bastante importante ao longo da tese.

Teorema 1.28 (Rosenthal). ([Di], pag. 82) Seja $\left(\mu_{n}\right)_{n \in \omega}$ uma sequência limitada em $M(K)$. Para todo $\varepsilon>0$ e toda sequência $\left(A_{n}\right)_{n \in \omega}$ de subconjuntos borelianos de $K$ dois a dois disjuntos, existe uma sequência estritamente crescente de inteiros $\left(k_{n}\right)_{n \in \omega}$ tal que

$$
\left|\mu_{k_{n}}\right|\left(\bigcup_{n \neq j}\left(A_{k_{j}}\right)\right)<\varepsilon
$$

para todo $n \in \omega$.

\subsection{A noção de poucos operadores em $C(K)$}

A noção de poucos operadores aparece em [Ko2] durante a construção de um espaço de Banach $C(K)$ indecomponível. Também em [Pl] a noção de poucos operadores aparece fortemente. Todos os resultados aqui apresentados são demonstrados em [Ko2], [Ko5] e [Ko6]. Também indicamos [Ko3] e [Ko4] para referências.

Definição 1.29. Dadas $f, g \in C(K)$ diremos que $f$ e $g$ são disjuntas se $f \cdot g=0$, isto é, se $f(x) \cdot g(x)=0$ para todo $x \in K$.

Ao longo de toda a tese utilizaremos o termo operador no sentido de operador linear contínuo. 
Definição 1.30. ([Ko2], 2.1) Um operador $T: C(K) \longrightarrow C(K)$ é um multiplicador fraco se para toda sequência limitada $\left(e_{n}: n \in \omega\right)$ de elementos dois a dois disjuntos de $C(K)$, e toda sequência $\left(x_{n}: n \in \omega\right) \subseteq K$ tal que $e_{n}\left(x_{n}\right)=0$, temos

$$
\lim _{n \rightarrow \infty} T\left(e_{n}\right)\left(x_{n}\right)=0
$$

Definição 1.31. ([Ko4], 1.1) Um operador $T: C(K) \longrightarrow C(K)$ é uma multiplicação fraca se pudermos escrever $T=g I+S$, onde $g \in C(K), I$ é o operador identidade em $C(K)$ e $S: C(K) \longrightarrow C(K)$ é fracamente compacto (equivalentemente, $S$ é estritamente singular).

Lema 1.32. ([Ko2], 2.3) Seja $T: C(K) \longrightarrow C(K)$ um multiplicador fraco. Então $T$ é um isomorfismo sobre a imagem se, e somente se, $T$ é sobrejetor em $C(K)$.

Teorema 1.33. ([Ko2], 2.4) Suponha que todos os operadores em $C(K)$ são multiplicadores fracos. Então $C(K)$ não é isomorfo a nenhum dos seus hiperplanos e $C(K)$ é um espaço de Grothendieck ${ }^{1}$.

Teorema 1.34. ([Ko2], 2.5) Suponha que todos os operadores em $C(K)$ são multiplicadores fracos e que $K \backslash F$ é conexo, para todo $F \subseteq K$ finito. Então $C(K)$ é um espaço de Banach indecomponível.

Definição 1.35. Sejam $X$ e $Y$ espaços topológicos tais que $Y \subseteq X$. Dizemos que $Y$ é $C^{*}$-imerso em $X$ se toda função contínua e limitada de $Y$ em $\mathbb{R}$ se estende a uma função contínua e limitada de $X$ em $\mathbb{R}$.

Definição 1.36. ([Pl], 2.3) Dizemos que um espaço topológico $K$ contém um aberto-borboleta se existem conjuntos abertos $U, V \subseteq K$ tais que $\bar{U} \cap \bar{V}$ é unitário.

Lema 1.37. ([Ko2], 2.8) Seja $K$ um espaço topológico compacto tal que, para todos $U_{1}$ e $U_{2}$ abertos disjuntos em $K, \bar{U}_{1} \cap \bar{U}_{2}=\emptyset$ ou $\left|\bar{U}_{1} \cap \bar{U}_{2}\right| \geq 2$. Então para todo $x \in K$ o espaço $K \backslash\{x\}$ é $C^{*}$-imerso em $K$.

\footnotetext{
${ }^{1}$ Dizemos que um espaço de Banach X é um espaço de Grothendieck se as convergências fraca e fraca* ${ }^{*}$ de sequências coincidem no espaço dual $X^{*}$.
} 
Teorema 1.38. ([Ko2], 2.7) Seja K um espaço topológico compacto. São equivalentes:

a) Todo operador $T: C(K) \longrightarrow C(K)$ é da forma $g I+S$, onde $g \in C(K)$ e $S$ é fracamente compacto.

b) Todos os operadores em $C(K)$ são multiplicadores fracos e, para todo $x \in K$, o espaço $K \backslash\{x\}$ é $C^{*}$-imerso em $K$.

Definição 1.39. Dizemos que o espaço $C(K)$ tem poucos operadores se todo operador em $C(K)$ for um multiplicador fraco.

Definição 1.40. Seja $K$ um espaço topológico compacto. Dizemos que:

1) $K$ é um espaço de Koszmider se todo operador em $C(K)$ é multiplicação fraca;

2) $K$ é um espaço hereditariamente Koszmider se para qualquer fechado $L \subseteq K$, todo operador em $C(L)$ é multiplicação fraca;

3) $K$ é um espaço fracamente Koszmider se todo operador em $C(K)$ é multiplicador fraco;

4) $K$ é um espaço hereditariamente fracamente Koszmider se para qualquer fechado $L \subseteq K$, todo operador em $C(L)$ é multiplicador fraco.

Observação: Schachermayer mostra, em [Sc], que um espaço de Banach $C(K)$ é de Grothendieck se, e somente se, não contém $c_{0}$ complementado. Pelo Teorema 1.33 temos que se $K$ é um espaço hereditariamente fracamente Koszmider então $K$ não contém cópia de $\beta \mathbb{N}$ pois $C(\beta \mathbb{N}) \equiv l_{\infty}$ e sabemos que $l_{\infty}$ é isomorfo aos seus hiperplanos. Além disso, $K$ não possui sequência convergente não trivial, pois do contrário teríamos $c_{0}$ complementado em $C(K)$.

Definição 1.41. A densidade de um espaço topológico $X, d(X)$, é o menor cardinal infinito $\kappa$ tal que $X$ possui um subconjunto denso de cardinalidade menor ou igual a $\kappa$. Se $d(X)=\omega$, dizemos que $X$ é separável. Da mesma maneira definimos a densidade de um espaço de Banach como sendo o menor 
cardinal infinito $\kappa$ para o qual existe um subconjunto denso de cardinalidade menor ou igual a $\kappa$, e diremos que o espaço de Banach é separável se $\kappa=\omega$.

O primeiro exemplo consistente de um espaço de Banach indecomponível de densidade maior que $2^{\omega}$ foi construído em [Ko5]. Utilizando o método do forcing, Koszmider obteve o seguinte resultado:

Teorema 1.42. ([Ko5], 1.1) Existe consistentemente com ZFC um espaço de Banach da forma $C(K)$ tal que:

a) A densidade de $C(K)$ é $2^{2^{\omega}}=\omega_{2}>\omega_{1}=2^{\omega}$;

b) Todo operador $T: C(K) \longrightarrow C(K)$ é da forma $g I+S$, onde $g \in C(K)$ e $S$ é fracamente compacto;

c) $C(K)$ é indecomponível.

Definição 1.43. A Hipótese Generalizada do Contínuo ${ }^{2}$, indicada por GCH, é a afirmação de que $\omega_{\alpha+1}=2^{\omega_{\alpha}}$ para todo ordinal $\alpha$.

Utilizando uma técnica diferente das construções clássicas dos espaços $C(K)$ com poucos operadores, Koszmider, Shelah e S̀wietek obtiveram recentemente o seguinte resultado:

Teorema 1.44. ([Ko6], 1.3) Assuma GCH. Para todo cardinal $\kappa$ existe um espaço de Banach indecomponível de densidade maior que $\kappa$. Além disso, os espaços são da forma $C(K)$ com poucos operadores.

\subsection{O método do forcing}

Seja $\Gamma$ um conjunto de fórmulas. Dizemos que $\Gamma$ é consistente se de $\Gamma$ não deduzirmos contradições, isto é, se não existir fórmula $\phi$ tal que $\Gamma \vdash \phi \wedge \neg \phi$. Do contrário, diremos que $\Gamma$ é inconsistente. Dada uma fórmula $\phi$, dizemos que $\phi$ é relativamente consistente com $\Gamma$ se a consistência de $\Gamma$ implicar a consistência de $\Gamma \cup\{\phi\}$. Isso equivale a dizer que de $\Gamma$ não podemos

\footnotetext{
${ }^{2}$ A Hipótese Generalizada do Contínuo é independente de ZFC. Indicamos [Je] e [Ku] para referências.
} 
deduzir $\neg \phi$, a menos que $\Gamma$ seja inconsistente. Além disso, uma fórmula $\phi$ é independente de $\Gamma$ se tanto $\phi$ quanto $\neg \phi$ forem relativamente consistentes com $\Gamma$. O método do forcing é um método utilizado para se demonstrar a consistência relativa de certas fórmulas. Esse método foi introduzido por Paul Cohen, em 1964, para se provar a consistência relativa da negação da hipótese do contínuo. Em 1940, o matemático Austríaco Kurt Gödel já havia demonstrado a consistência relativa da hipótese do contínuo e, portanto, segue que o problema do contínuo de Cantor, o primeiro dos 23 Problemas de Hilbert de 1900, é indecidível (veja [Go]). Na exposição abaixo seguiremos a notação e o método de [Ku], e todas as demonstrações dos resultados aqui apresentados podem ser vistos em $[\mathrm{Ku}]$ ou [Je].

Lembramos que o sistema de axiomas usual para a matemática é o sistema de Zermelo-Frankel com o axioma da escolha (ZFC). Além disso, lembramos também que a linguagem de ZFC é formada pelos seguintes símbolos: os quantificadores $\forall \mathrm{e} \exists$, os conectivos lógicos $\neg, \wedge, \vee, \rightarrow \mathrm{e} \leftrightarrow$, a igualdade $=$, a relação de pertinência $\in$, os delimitadores ( e ), e as variáveis $x_{1}, x_{2}, x_{3}, \ldots$

Definição 1.45. Um modelo para a teoria dos conjuntos é um par $(M, \varepsilon)$ onde $M$ é um conjunto e $\varepsilon \subseteq M \times M$ é uma relação binária. Dizemos que o modelo é standard se a relação $\varepsilon$ coincide com a relação de pertinência $\epsilon$, isto é, se $\varepsilon=\{(x, y) \in M: x \in y\}$. Dizemos também que um modelo standard é transitivo se $x \subseteq M$, para todo $x \in M$.

Ao longo da tese iremos trabalhar apenas com modelos standard.

Definição 1.46. ([Ku], IV, 2.1) Seja $M$ uma classe. Para cada fórmula $\phi$ definimos a relativização de $\phi$ a $M$, que será denotada por $\phi^{M}$, por indução na complexidade de $\phi$ da seguinte maneira:

1. $(x=y)^{M}$ é $x=y$;

2. $(x \in y)^{M}$ é $x \in y$;

3. $(\phi \wedge \psi)^{M}$ é $\phi^{M} \wedge \psi^{M}$;

4. $(\neg \phi)^{M}$ é $\neg\left(\phi^{M}\right)$; 
5. $(\exists x \phi)^{M}$ é $\exists x\left(x \in M \wedge \phi^{M}\right)$.

Diremos que $M$ satisfaz $\phi$ ou que $\phi$ vale em $M$, e denotaremos por $M \models \phi$, se vale $\phi^{M}$.

Pelo Teorema da Incompletude de Gödel, sabemos que ZFC não pode provar a sua própria consistência e, portanto, não podemos provar, em ZFC, que existe um modelo para ZFC. O Teorema de Löwenheim-Skolem garante a existência de um modelo enumerável para qualquer teoria consistente. Mas, para uso do forcing, precisamos de um modelo transitivo. Além disso, ainda que ZFC seja consistente, pode ser que não exista um modelo transitivo para ZFC, pois ZFC não é finitamente axiomátizavel. Entretanto, sabemos que demonstrações usam uma quantidade finita de fórmulas e, além disso, sabemos que se $\Gamma$ é um conjunto finito e consistente de fórmulas então existe modelo transitivo enumerável $M$ tal que $M \models \Gamma$ (veja [Ku]). Temos portanto o seguinte resultado, conhecido como o Princípio do Forcing.

Teorema 1.47 (Princípio do forcing). Para mostrar que uma fórmula $\varphi$ é relativamente consistente com ZFC é suficiente mostrar que, para todo modelo transitivo enumerável $M$ que satisfaz $Z F C$, existe um modelo $N$ que satisfaz ZFC tal que $M \subseteq N$ e $N \models \varphi$.

Definição 1.48. Sejam $\mathbb{P}=(P, \leq)$ um conjunto parcialmente ordenado e $p, q \in \mathbb{P}$ elementos quaisquer. Dizemos que:

i) $p$ estende $q$, ou ainda, $p$ é uma extensão de $q$, se $p \leq q$;

ii) $p$ e $q$ são compatíveis se existe $r \in \mathbb{P}$ que estende $p$ e $q$. Do contrário, diremos que $p$ e $q$ são incompatíveis, e escreveremos $p \perp q$.

Definição 1.49. Um conjunto parcialmente ordenado $\mathbb{P}=(P, \leq)$ será chamado de forcing se satisfizer as seguintes duas condições:

i) Para todo $p \in \mathbb{P}$ existem $q_{1}, q_{2} \in \mathbb{P}$ incompatíveis que estendem $p$, isto é, $q_{1}, q_{2} \leq p$ e $q_{1} \perp q_{2}$;

ii) Existe o elemento máximo de $\mathbb{P}$, que denotaremos por $\mathbf{1}_{\mathbb{P}}$. 
Se $\mathbb{P}=(P, \leq)$ for um forcing, os elementos de $P$ serão chamados de condições do forcing.

Definição 1.50. Seja $\mathbb{P}$ um forcing. Dizemos que um subconjunto $D \subseteq \mathbb{P}$ é denso em $\mathbb{P}$ se para todo $p \in \mathbb{P}$ existe $q \in D$ tal que $q \leq p$. Dizemos também que um subconjunto $A \subseteq \mathbb{P}$ é uma anticadeia em $\mathbb{P}$ se os elementos de $A$ são dois a dois incompatíveis.

Definição 1.51. Sejam $\mathbb{P}$ um forcing e $G \subseteq \mathbb{P}$ um subconjunto não vazio. Dizemos que $G$ é um filtro em $\mathbb{P}$ se :

i) Dados $p, q \in G$ existe $r \in G$ tal que $r \leq p$ e $r \leq q$;

ii) Para todo $p \in G$ e todo $q \in \mathbb{P}$, se $p \leq q$ então $q \in G$.

Definição 1.52. Sejam $M$ um modelo transitivo enumerável para ZFC e $\mathbb{P} \in M$ um forcing. Dizemos que um subconjunto $G \subseteq \mathbb{P}$ é um $\mathbb{P}$-genérico sobre $M$ se $G$ é um filtro em $\mathbb{P}$ e, para todo $D \in M$ denso em $\mathbb{P}$, temos $G \cap D \neq \emptyset$.

Lema 1.53. ([Ku], VII, 2.3) Sejam $M$ um modelo transitivo enumerável para $Z F C$ e $\mathbb{P} \in M$ um forcing. Então, para todo $p \in \mathbb{P}$ existe $G$ um filtro $\mathbb{P}$-genérico sobre $M$ tal que $p \in G$.

Observe que o $\mathbb{P}$-genérico $G$ dado pelo Lema 1.53 não pertence a $M$. Com efeito, suponha que $G \in M$ e defina $D=\mathbb{P} \backslash G$. Como $\mathbb{P}, G \in M$ e $M$ é modelo transitivo para ZFC, teríamos também que $D \in M$. Seja agora $p \in \mathbb{P}$ qualquer. Pela definição de forcing, existem $q, r \in \mathbb{P}$ tais que $q, r \leq p$ e $q \perp r$. Como $G$ é um filtro, temos que ou $q \notin G$ ou $r \notin G$, pois quaisquer dois elementos de $G$ são compatíveis. Logo, segue que ao menos uma das extensões está em $D$. Isto nos mostra que $D$ é denso. Mas também temos $D \cap G=\emptyset$, contradição com o fato de $G$ ser $\mathbb{P}$-genérico sobre $M$.

Para se provar a consistência relativa de uma fórmula $\phi$ usando o método do forcing, partimos de um modelo transitivo enumerável para ZFC, que podemos assumir que existe, pelo princípio do forcing. Em seguida, tomamos $G$ um $\mathbb{P}$-genérico sobre $M$ e olhamos para $M[G]$, que será o menor modelo 
transitivo enumerável para ZFC que contém $M$ e que satisfaz $G \in M[G]$. O genérico $G$ será adicionado a $M$ para se "forçar" a fórmula $\phi$, isto é, para que tenhamos $M[G] \models \phi$.

Definição 1.54. Seja $\mathbb{P}$ um forcing. Diremos que $\tau$ é um $\mathbb{P}$-nome se $\tau$ é um conjunto de pares ordenados da forma $(\sigma, p)$, onde $p \in \mathbb{P}$ e $\sigma$ é um $\mathbb{P}$-nome. Denotamos por $V^{\mathbb{P}}$ a classe de todos os $\mathbb{P}$-nomes. Além disso, se $M$ é um modelo transitivo enumerável para ZFC, com $\mathbb{P} \in M$, definimos $M^{\mathbb{P}}=M \cap V^{\mathbb{P}}$ o conjunto de todos os $\mathbb{P}$-nomes sobre $\mathrm{M}$.

Observação. Para se formalizar a definição de $\mathbb{P}$-nomes, usamos o fato de que a relação de pertinência $\in$ é bem-fundada e o Teorema da recursão sobre relações bem-fundadas (veja $[\mathrm{Ku}]$ ). Intuitivamente, o que estamos fazendo funciona como uma definição recursiva sobre $\omega$, ou sobre um cardinal qualquer. O conjunto $\emptyset$ é um $\mathbb{P}$-nome. Logo, $\{(\emptyset, p): p \in \mathbb{P}\}$ também é um $\mathbb{P}$-nome, bem como $\{((\emptyset, p), q): p, q \in \mathbb{P}\}$, e assim por diante. Convém observar também que estamos usando a noção informal de classes, apenas como uma notação. Dizer portanto que $\tau \in V^{\mathbb{P}}$ significa dizer que $\tau$ é um $\mathbb{P}$-nome. Para uma formalização da noção de classes, indicamos o estudo das teorias NGB (Von Neumann-Bernays-Gödel) e KM (Kelley-Morse). Várias outras definições que daremos a seguir usam o Teorema da recursão sobre relações bem-fundadas.

Definição 1.55. Sejam $\mathbb{P}$ um forcing, $G$ um filtro e $\tau$ um $\mathbb{P}$-nome. Definimos $\operatorname{val}_{G}(\tau)$ como sendo o conjunto $\left\{\operatorname{val}_{G}(\sigma): \exists p \in G(\sigma, p) \in \tau\right\}$. Seja $M$ um modelo transitivo enumerável para ZFC, com $\mathbb{P} \in M$, e suponha que $\mathrm{G}$ é um $\mathbb{P}$-genérico sobre $M$. Definimos $M[G]=\left\{\operatorname{val}_{G}(\tau): \sigma \in M^{\mathbb{P}}\right\}$.

Definição 1.56. Seja $\mathbb{P}$ um forcing. Definimos o $\mathbb{P}$-nome $\check{x}$ recursivamente por $\check{x}=\left\{\left(\check{y}, \mathbf{1}_{\mathbb{P}}\right): y \in x\right\}$. Definimos também o nome $\Gamma=\{(\check{p}, p): p \in \mathbb{P}\}$.

Lema 1.57. ([Ku], VII, 2.11 e 2.13) Sejam $M$ um modelo transitivo enumerável para $Z F C, \mathbb{P} \in M$ um forcing e $G$ um $\mathbb{P}$-genérico sobre $M$. Então

a) Para todo $x \in M, \check{x} \in M^{\mathbb{P}}$ e $\operatorname{val}_{G}(\check{x})=x$; 
b) $\operatorname{val}_{G}(\Gamma)=G$.

Note que o lema anterior nos garante que $M \subseteq M[G]$ e $G \in M[G]$. O teorema a seguir, essencial no método do forcing, nos trará todas as boas propriedades de $M[G]$.

Teorema 1.58. ([Ku], VII, 2.14, 4.2, 2.11, 2.13, 2.19) Sejam $M$ um modelo transitivo enumerável para $Z F C, \mathbb{P}$ um forcing tal que $\mathbb{P} \in M$ e $G$ um $\mathbb{P}$-genérico sobre $M$. Então

a) $M[G]$ é um modelo transitivo enumerável;

b) $M[G] \models Z F C$;

c) $M \subseteq M[G]$;

d) $G \in M[G]$;

e) Se $N$ é um modelo transitivo para $Z F C$ tal que $N \supseteq M$ e $G \in N$, então $M[G] \subseteq N$.

Iremos agora definir a relação de forcing $\Vdash$, que nos permitirá entender de que maneira os elementos de $\mathbb{P}$ fornecem informações sobre verdades em $M[G]$, de modo que, se escolhermos convenientemente $\mathrm{G}$, a fórmula $\phi$ vale em $M[G]$. De fato, veremos a seguir que dados $M$ e $G$, uma fórmula $\phi$ vale em $M[G]$ se, e somente se, existe $p \in G$ tal que $p \Vdash \phi$.

Sejam $M$ um modelo transitivo enumerável para ZFC e $\mathbb{P}$ um forcing em $M$. Definimos a linguagem do forcing $\mathbb{P}$ sobre $M$, que será denotada por $\mathcal{L}\left(M^{P}\right)$, como a linguagem da teoria dos conjuntos substituindo as variáveis livres por $\mathbb{P}$-nomes sobre $M$.

Teorema 1.59. ([Je], 14.6 e 14.7) Sejam $M$ um modelo transitivo enumerável para $Z F C$ e $\mathbb{P} \in M$ um forcing. Existe uma relação $\Vdash \subseteq P \times \mathcal{L}\left(M^{P}\right)$ tal que:

(i) $\Vdash \in M$;

(ii) (a) $p \Vdash \phi\left(\tau_{1}, \ldots, \tau_{n}\right)$ se, e somente se, $M[G] \models \phi\left(\operatorname{val}_{G}\left(\tau_{1}\right), \ldots, v_{a l}\left(\tau_{n}\right)\right)$, para todo $G$ filtro $\mathbb{P}$-genérico sobre $M$ tal que $p \in G$; 
(b) $M[G] \models \phi\left(\operatorname{val}_{G}\left(\tau_{1}\right), \ldots\right.$, val $\left._{G}\left(\tau_{n}\right)\right)$ se, e somente se, existe $p \in G$ tal que $p \Vdash \phi\left(\tau_{1}, \ldots, \tau_{n}\right)$;

(iii) (a) Se $p \Vdash \phi$ e $q \leq p$ então $q \Vdash \phi$;

(b) Não existem $p \in \mathbb{P}$ e $\phi \in \mathcal{L}\left(M^{P}\right)$ tais que $p \Vdash \phi$ e $p \Vdash \neg \phi$;

(c) Para todos $p \in \mathbb{P}$ e $\phi \in \mathcal{L}\left(M^{P}\right)$ existe $q \leq p$ tal que $q \Vdash \phi$ ou $q \Vdash \neg \phi ;$

(d) $p \Vdash \neg \phi$ se, e somente se, $q \Downarrow \phi$, para todo $q \leq p$;

(e) $p \Vdash \phi \wedge \psi$ se, e somente se, $p \Vdash \phi e p \Vdash \psi$;

(f) $p \Vdash \exists x \phi(x)$ se, e somente se, existe $\tau \in M^{P}$ tal que $p \Vdash \phi(\tau)$.

(iv) $p \Vdash$ " $\Gamma$ é um filtro" e, se $D$ é denso em $P$, então $p \Vdash \Gamma \cap \check{D} \neq \emptyset$.

Definição 1.60. A relação $\Vdash$ é chamada relação de forcing. Se $p \Vdash \phi$, diremos que $p$ força $\phi$.

Definição 1.61. ([Ku], IV, 3.1) Sejam $\phi$ uma fórmula com variáveis livres $x_{1}, \ldots, x_{n}$ e $M \subseteq N$ modelos transitivos para ZFC. Dizemos que

1. $\phi$ é absoluta em relação a $M$ e $N$ se

$$
\forall x_{1}, \ldots, x_{n} \in M\left(M \models \phi\left(x_{1}, \ldots, x_{n}\right) \leftrightarrow N \models \phi\left(x_{1}, \ldots, x_{n}\right)\right) ;
$$

2. $\phi$ é absoluta em relação a $M$ se

$$
\forall x_{1}, \ldots, x_{n} \in M\left(M \models \phi\left(x_{1}, \ldots, x_{n}\right) \leftrightarrow \phi\left(x_{1}, \ldots, x_{n}\right)\right)
$$

3. $\phi$ é absoluta se é absoluta em relação a todo modelo transitivo.

O lema a seguir nos traz algumas fórmulas que são absolutas. Note que, quando escrevemos que $\omega$ é absoluto queremos dizer que a fórmula " $x$ é o primeiro ordinal infinito" é absoluta. Além das fórmulas listadas abaixo, um outro grupo de fórmulas absolutas são as fórmulas $\Delta_{0}$. Essas fórmulas são definidas indutivamente através das seguintes regras: $x \in y$ e $x=y$ são $\Delta_{0}$ e, se $\phi$ e $\varphi$ são $\Delta_{0}$, então $\neg \phi, \phi \wedge \varphi$ e $\exists x(x \in y \wedge \phi)$ são $\Delta_{0}$ (veja $[\mathrm{Ku}]$ ). 
Lema 1.62. ([Ku], IV, 5.1 e 5.3) As seguintes fórmulas são absolutas:

1. $\omega ;$

2. $x$ é um ordinal;

3. $x$ é um ordinal limite;

4. $x$ é um ordinal sucessor;

5. $x$ é finito.

Ainda que "ser um ordinal" seja absoluto para modelos transitivos, o mesmo não ocorre com "ser um cardinal". Isso significa dizer que, dado um cardinal $\kappa \in M$, quando olhamos para $\kappa$ em $M[G]$ teremos um ordinal que não sabemos se é, ou não, um cardinal. Isso motiva a seguinte definição:

Definição 1.63. Dizemos que um forcing $\mathbb{P}$ preserva cardinais se para todo modelo transitivo enumerável $M$ tal que $\mathbb{P} \in M$, todo cardinal $\kappa \in M$, e todo filtro $\mathbb{P}$-genérico $G$ sobre $M$, tivermos que $\kappa$ é um cardinal em $M[G]$.

As próximas duas definições e o próximo teorema são úteis sempre que quisermos mostrar que um forcing $\mathbb{P}$ preserva cardinais.

Definição 1.64. Seja $\kappa$ um cardinal. Dizemos que um forcing $\mathbb{P}$ é $\kappa$-fechado se para toda cadeia $\left(p_{\alpha}\right)_{\alpha<\kappa} \subset \mathbb{P}$ decrescente, existe $p \in \mathbb{P}$ tal que $p \leq p_{\alpha}$, para todo $\alpha<\kappa$. Além disso, dizemos que um forcing $\mathbb{P}$ é $\sigma$-fechado se é $\omega_{1}$-fechado.

Definição 1.65. Seja $\kappa$ um cardinal. Dizemos que um forcing $\mathbb{P}$ é $\kappa$-c.c se toda anticadeia em $\mathbb{P}$ tem cardinalidade menor que $\kappa$.

Teorema 1.66. ([Ku], VII, 6.9 e 6.15) Seja $\kappa$ um cardinal. Se um forcing $\mathbb{P}$ é $\kappa$-fechado e $\kappa^{+}$-c.c, então $\mathbb{P}$ preserva cardinais. Em particular, todo forcing $\sigma$-fechado e $\omega_{2}$-c.c preserva cardinais. 


\section{Capítulo 2}

\section{Extensões por funções contínuas}

\subsection{Introdução}

Em [Ko2], Koszmider construiu um espaço topológico compacto totalmente desconexo $K$ tal que todo operador $T$ em $C(K)$ é da forma $T=g I+S$, onde $I: C(K) \longrightarrow C(K)$ representa o operador identidade, $g \in C(K)$ e $S: C(K) \longrightarrow C(K)$ é um operador fracamente compacto. O espaço $K$ foi obtido como o Espaço de Stone de uma Álgebra de Boole contruída por indução transfinita, onde cada $\mathcal{A}_{\alpha+1}$ é a Álgebra de Boole gerada por $\mathcal{A}_{\alpha} \mathrm{e}$ o supremo de uma anticadeia em $\mathcal{A}_{\alpha}$. No passo limite tomamos a união.

O mesmo artigo provou também a existência de um Espaço de Banach indecomponível da forma $C(K)$. A construção é similar à anterior, exceto pelo fato de $K$ ser conexo. Dessa forma, $K$ não pode ser o Espaço de Stone de uma Álgebra de Boole. A ideia central na construção de tal $K$ foi trocar o supremo de elementos da Álgebra de Boole - que corresponde, no Espaço de Stone, ao supremo das funções características de tais elementos - pelo supremo de funções contínuas disjuntas. E nesta hora, Koszmider desenvolveu uma nova técnica conhecida como extensões por funções contínuas.

Antes de introduzirmos a particular construção de Koszmider, vamos apresentar uma visão geral do problema. Seja $K$ um espaço topológico com- 
pacto. Defina por $C(K)$ o Espaço de Banach de todas as funções contínuas em $K$, munido da norma do supremo, e $C_{1}(K)$ o conjunto de todas as funções contínuas de $K$ em [0,1].

Seja $\left(f_{n}\right)_{n \in \mathbb{N}}$ uma sequência de funções duas a duas disjuntas em $C_{1}(K)$. Sejam $L$ outro espaço topológico compacto e $\pi$ uma função contínua e sobrejetora de $L$ em $K$. Diremos que $(L, \pi)$ adiciona o supremo de $\left(f_{n}\right)_{n \in \mathbb{N}}$ se $\sup \left\{f_{n} \circ \pi: n \in \mathbb{N}\right\}$ existir em $C(L)$. Além disso, diremos que $(L, \pi)$ preserva supremos se $\sup \left\{g_{n} \circ \pi: n \in \mathbb{N}\right\}$ existir em $C(L)$, sempre que $\left(g_{n}\right)_{n \in \mathbb{N}}$ é uma sequência disjunta em $C_{1}(K)$ que tem supremo em $C(K)$.

Vamos agora ver como isto funciona no caso das Álgebras de Boole. Sejam $\mathcal{A}$ uma Álgebra de Boole e $\left(a_{n}\right)_{n \in \mathbb{N}}$ uma sequência de elementos dois a dois disjuntos em $\mathcal{A}$. Seja $b$ o supremo de $\left(a_{n}\right)_{n \in \mathbb{N}}$ no completamento de $\mathcal{A}$. Tome $\mathcal{B}$ a álgebra gerada por $\mathcal{A} \cup\{b\}$.

Sejam $S(\mathcal{A})$ e $S(\mathcal{B})$ os espaços de Stone de $\mathcal{A}$ e $\mathcal{B}$, respectivamente. Seja $\pi$ a projeção standard de $S(\mathcal{B})$ sobre $S(\mathcal{A})$, dada por $\pi(u)=u \cap \mathcal{A}$, onde $u$ é um ultrafiltro em $\mathcal{B}$.

Para cada $a \in \mathcal{B}$, denotaremos por $[a]_{\mathcal{B}}$ o conjunto aberto-fechado de $S(\mathcal{B})$ formado por todos os ultrafiltros sobre $\mathcal{B}$ que contêm $a$. Se $a \in \mathcal{A}$, a notação $[a]_{\mathcal{A}}$ significará o conjunto de todos os ultrafiltros sobre $\mathcal{A}$ que contêm $a$. É fácil ver que $\pi\left[[a]_{\mathcal{B}}\right]=[a]_{\mathcal{A}}$.

Em $C(S(\mathcal{B}))$ considere $\chi_{[b]_{\mathcal{B}}}$ a função característica de $[b]_{\mathcal{B}}$, que é contínua pelo fato de $[b]_{\mathcal{B}}$ ser um conjunto aberto-fechado. Como $b$ é o supremo de $\left(a_{n}\right)_{n \in \mathbb{N}}, \chi_{[b]_{\mathcal{B}}}$ é claramente o supremo de $\left\{\chi_{\left[a_{n}\right]_{\mathcal{B}}}: n \in \mathbb{N}\right\}$. Para cada $n \in \mathbb{N}$ temos $\chi_{\left[a_{n}\right]_{\mathcal{B}}}=\chi_{\left[a_{n}\right]_{\mathcal{A}}} \circ \pi$. Podemos então concluir que $(S(\mathcal{B}), \pi)$ adiciona o supremo de $\left(\chi_{\left[a_{n}\right]_{\mathcal{B}}}\right)_{n \in \mathbb{N}}$.

Se $\left(a_{n}^{\prime}\right)_{n \in \mathbb{N}}$ tem supremo $a$ em $\mathcal{A}$, então $\chi_{[a]_{\mathcal{B}}}$ é o supremo de $\left(\chi_{\left[a_{n}^{\prime}\right]_{\mathcal{B}}}\right)_{n \in \mathbb{N}}$ em $C(S(\mathcal{B}))$. Logo, $S(\mathcal{B})$ preserva supremos de funções deste tipo, i.e., funções características de abertos-fechados básicos. Para o caso geral, segue do Lema 4.1 de $[\mathrm{Ko} 2]$ que $(C(S(\mathcal{B})), \pi)$ preserva supremos.

Adicionar supremos de elementos de uma Álgebra de Boole é muito mais fácil que adicionar supremos em $C(K)$. Dessa forma, a melhor abordagem para adicionar supremos em $C(K)$ para $K$ totalmente desconexo é via Álgebras de Boole. Se $K$ é conexo, Koszmider, em [Ko2], introduziu a noção 
de extensões por funções contínuas para obter um espaço topológico compacto $L \subset K \times[0,1]$ que adiciona supremo de uma dada sequência disjunta $\left(f_{n}\right)_{n \in \mathbb{N}}$ em $C_{1}(K)$. A função $\pi$ é a projeção standard sobre $K$. Utilizaremos a notação $K\left(\left(f_{n}\right)_{n \in \mathbb{N}}\right)$ para indicar a extensão de $K$ por $\left(f_{n}\right)_{n \in \mathbb{N}}$.

A noção de extensões por funções contínuas foi aplicada em muitos contraexemplos na Teoria dos Espaços de Banach da forma $C(K)$, como acontece em [Ko2], [Fa1] e [Fa2]. Caminhos alternativos de adicionar supremos em espaços conexos foram desenvolvidos em [Pl] utilizando o teorema da representação de Wallman, que generaliza o teorema da representação de Stone para reticulados conexos.

A maior dificuldade nas construções que utilizam extensões por funções contínuas é assegurar a conexidade. Para isso, precisamos, eventualmente, passar a uma subsequência (como acontece em [Ko2]) ou modificar as funções (como acontece em [Fa2]).

O objetivo principal desse capítulo será apresentar três novos resultados envolvendo a técnica de extensões por funções contínuas. Com efeito, estudando a preservação de conexidade nas extensões por funções contínuas, obtivemos os seguintes resultados:

- Se $K$ é um espaço topológico compacto conexo, localmente conexo e metrizável, então toda extensão de $K$ por funções contínuas é conexa (mas pode não ser localmente conexa);

- Existe uma extensão desconexa de um espaço topológico compacto conexo e metrizável $K$;

- Para todo espaço topológico compacto metrizável $K$ existe um desconexo $L$ que é obtido a partir de $K$ por uma quantidade finita de extensões por funções contínuas.

Definição 2.1. Um espaço topológico metrizável, compacto e conexo será chamado de continuum.

Problema 1. Suponha que $K$ seja um continuum e que L seja uma extensão de $K$ por uma sequência de funções contínuas e duas a duas disjuntas $\left(f_{n}\right)_{n \in \mathbb{N}} \subseteq C_{1}(K)$. Será L necessariamente conexo? 
No Teorema 2.15 construíremos um continuum $K$ e uma sequência de funções duas a duas disjuntas $\left(f_{n}\right)_{n \in \mathbb{N}} \subseteq C_{1}(K)$ tal que $K\left(\left(f_{n}\right)_{n \in \mathbb{N}}\right)$ é desconexo. O Teorema 2.16 fornece uma adaptação da técnica utilizada no Teorema 2.15 de modo que $K$ seja ele mesmo uma extensão do intervalo $[0,1]$.

Uma vez respondido negativamente o Problema 1, podemos nos perguntar o que acontece se trocarmos a conexidade por alguma outra propriedade mais forte. Surge então a seguinte questão:

Problema 2. Existe alguma propriedade não-vazia $P$ na classe dos continua tal que, para todo $K$ satisfazendo $P$ e toda sequência de funções duas a duas disjuntas $\left(f_{n}\right)_{n \in \mathbb{N}} \subseteq C_{1}(K)$, então $K\left(\left(f_{n}\right)_{n \in \mathbb{N}}\right)$ também satisfaz P?

Na tentativa de responder o Problema 2, poderíamos considerar a propriedade $P$ como sendo conexidade local. Com efeito, no Teorema 2.14 provamos que se $K$ é um espaço topológico compacto localmente conexo e metrizável, então toda extensão de $K$ por funções contínuas é conexa. Mas a extensão pode não ser localmente conexa.

O Problema 2 também será respondido negativamente. O Teorema 2.18 prova que, começando com qualquer continuum $K_{0}$, existe uma sequência $\left(K_{i}\right)_{0 \leq i \leq 3}$ de espaços topológicos compactos tal que cada $K_{i}$ é uma extensão de $K_{i-1}$ por uma sequência de funções contínuas e $K_{3}$ é desconexo.

Assumindo a Hipótese do Contínuo, os problemas 1 e 2 podem ser respondidos indiretamente (com a hipótese adicional de que $P$ é preservado por limite inverso, no problema 2) usando a construção em [Ko2] e um Teorema que pode ser encontrado em [Me]. Se um desses problemas possuísse resposta afirmativa poderíamos construir, como em [Ko2], um espaço topológico compacto e conexo $K$ tal que toda sequência disjunta em $C_{1}(K)$ tem supremo. Por $[\mathrm{Me}]$ isso implica que $K$ é quasi-Stoneano ${ }^{1}$ e portanto desconexo. No entanto, vamos apresentar exemplos que mostram como a conexidade é perdida em extensões sucessivas por funções contínuas.

O principal teorema deste capítulo está intimamente relacionado com a

\footnotetext{
${ }^{1}$ Um espaço topológico compacto é quasi-Stoneano se o fecho de todo aberto $F_{\sigma}$ é aberto.
} 
Teoria dos Espaços de Banach com poucos operadores, já que refuta uma conjectura que poderia simplificar várias construções.

Observação. Ao longo de todo o capítulo utilizaremos a notação $G r(f)$ para indicar o gráfico de uma função $f$.

\subsection{Definições e lemas principais}

Nesta seção apresentamos as principais definições e os principais lemas envolvendo as extensões por funções contínuas, com base em [Ko2]. Se $f$ é uma função real definida sobre um espaço topológico compacto $K$, denotaremos por $\operatorname{supp}(f)$ o fecho de $\{x \in K: f(x) \neq 0\}$ em $K$.

Definição 2.2. Sejam $K$ um espaço topológico compacto e $\left(f_{n}\right)_{n \in \mathbb{N}}$ uma sequência em $C_{1}(K)$. Definimos

$$
D\left(\left(f_{n}\right)_{n \in \mathbb{N}}\right)=\bigcup\left\{U: U \text { é aberto e }\left\{n: U \cap \operatorname{supp}\left(f_{n}\right) \neq \emptyset\right\} \text { é finito }\right\} .
$$

Lema 2.3. ([Ko2], 4.1) Seja $K$ um espaço topológico compacto e seja $\left(f_{n}\right)_{n \in \mathbb{N}} \subseteq C_{1}(K)$ uma sequência de funções duas a duas disjuntas. Então:

(i) $f \in C(K)$ é $\sup \left\{f_{n}: n \in \mathbb{N}\right\}$ no reticulado $C(K)$ se, e somente se,

$$
\left\{x \in K: \Sigma_{n \in \mathbb{N}} f_{n}(x) \neq f(x)\right\}
$$

é $\operatorname{raro}^{2}$ em $K$;

(ii) $D\left(\left(f_{n}\right)_{n \in \mathbb{N}}\right)$ é um subconjunto aberto-denso de $K$ e $\Sigma_{n \in \mathbb{N}} f_{n}$ é contínua em $D\left(\left(f_{n}\right)_{n \in \mathbb{N}}\right)$.

Definição 2.4. Sejam $K$ um espaço topológico compacto, $L \subseteq K \times[0,1]$ e $\left(f_{n}\right)_{n \in \mathbb{N}} \subseteq C_{1}(K)$ uma sequência de funções duas a duas disjuntas. Dizemos que $L$ é uma extensão de $K$ por $\left(f_{n}\right)_{n \in \mathbb{N}}$, e denotaremos por $K\left(\left(f_{n}\right)_{n \in \mathbb{N}}\right)$, se $L$ é o fecho do gráfico de $\Sigma_{n \in \mathbb{N}} f_{n} \mid D\left(\left(f_{n}\right)_{n \in \mathbb{N}}\right)$. Dizemos que $L$ é uma extensão forte se, além disso, contém o gráfico de $\Sigma_{n \in \mathbb{N}} f_{n}$.

\footnotetext{
${ }^{2}$ Dizemos que um subconjunto $M$ de um espaço topológico $K$ é raro se seu fecho tem interior vazio, isto é, se não existe um aberto $V$ não-vazio tal que $V \subseteq \bar{M}$.
} 
Definição 2.5. Sejam $K$ um espaço topológico compacto e $L \subseteq K \times[0,1]$ uma extensão de $K$. Dizemos que $L$ é uma extensão completa se para todo $x \in K, \pi_{L, K}^{-1}[\{x\}]$ é unitário ou igual a $[0,1] \times\{x\}$.

Lema 2.6. ([Fa2], 3.6) Seja $K$ um espaço topológico compacto e conexo. Se $L$ é uma extensão completa de $K$, então L também é conexo.

Lema 2.7. ([Ko2], 4.3 e 4.4) Seja $\left(f_{n}\right)_{n \in \mathbb{N}} \subseteq C_{1}(K)$ uma sequência de funções duas a duas disjuntas. Tome $L=K\left(\left(f_{n}\right)_{n \in \mathbb{N}}\right)$ e $\pi: L \longrightarrow K a$ projeção standard. Então $(L, \pi)$ adiciona o supremo de $\left(f_{n}\right)_{n \in \mathbb{N}}$ e preserva supremos. Além disso, se $L$ é uma extensão forte e $K$ é conexo, então $L$ também é conexo.

Em [Ko2], Koszmider prova que a projeção $\pi$ preserva conjuntos raros, i.e., $\pi^{-1}[M]$ é raro em $L$ sempre que $M$ for raro em $K$. Logo, a preservação de supremos segue do lema 2.3.

O supremo de $\left(f_{n} \circ \pi\right)_{n \in \mathbb{N}}$ em $L$ é a projeção na segunda coordenada, i.e., a função $f$ definida como $f(x, t)=t$, para $(x, t) \in L \subset K \times[0,1]$.

O próximo lema é uma versão simplificada do Lema 4.5 de [Ko2]. Ele mostra que a extensão é forte para muitas subsequências de $\left(f_{n}\right)_{n \in \mathbb{N}}$.

Lema 2.8. ([Ko2], 4.5) Seja $K$ um espaço topológico compacto de peso $\kappa<2^{\omega}$. Suponha que $\left(f_{n}\right)_{n \in \mathbb{N}} \subseteq C_{1}(K)$ seja uma sequência de funções duas a duas disjuntas e que $\left(N_{\xi}\right)_{\xi<2^{\omega}}$ seja uma família de subconjuntos infinitos de $\mathbb{N}$ tal que $N_{\xi} \cap N_{\xi^{\prime}}$ é finito, para todo $\xi \neq \xi^{\prime}$. Então existe $A \subset 2^{\omega}$ de cardinalidade não maior que $\kappa$ tal que, para todo $\xi \in 2^{\omega} \backslash A$ e todo $b \subset N_{\xi}$ infinito, a extensão de $K$ por $\left(f_{n}\right)_{n \in b}$ é forte.

Em [Fa2], Fajardo encontra algumas maneiras de obter a conexidade na extensão modificando ligeiramente as funções $f_{n}$. O Lema 2.11 nos mostra que a extensão é completa para muitas subsequências de $\left(f_{n}\right)_{n \in \mathbb{N}}$.

Definição 2.9. Se $K$ é um espaço compacto e $\left(f_{n}\right)_{n \in \mathbb{N}} \subseteq C_{1}(K)$ é uma sequência de funções duas a duas disjuntas, denotaremos por $\Delta\left(\left(f_{n}\right)_{n \in \mathbb{N}}\right)$ o conjunto $K \backslash D\left(\left(f_{n}\right)_{n \in \mathbb{N}}\right)$. 
Lema 2.10. ([Fa2], 3.6 e 3.8) Seja $K$ um continuum. Suponha que $\left(\varepsilon_{n}\right)_{n \in \mathbb{N}}$ é uma sequência de reais positivos, $\left(f_{n}\right)_{n \in \mathbb{N}} \subseteq C_{1}(K)$ é uma sequência de funções duas a duas disjuntas, $\left(\mu_{n}\right)_{n \in \mathbb{N}}$ é uma sequência de medidas regulares sobre $K$ e $\left(x_{n}\right)_{n \in \mathbb{N}}$ é uma sequência de pontos em $K$ tal que $f_{n}\left(x_{n}\right)=1$. Então existe uma sequência $\left(f_{n}^{\prime}\right)_{n \in \mathbb{N}}$ em $C_{1}(K)$ tal que

(i) Para todo $n \in \mathbb{N}, \operatorname{supp}\left(f_{n}^{\prime}\right) \subset \operatorname{supp}\left(f_{n}\right), f_{n}^{\prime}\left(x_{n}\right)=1 e$ $\int\left|f_{n}^{\prime}-f_{n}\right| d\left|\mu_{n}\right|<\varepsilon_{n}$

(ii) $K\left(\left(f_{n}^{\prime}\right)_{n \in \mathbb{N}}\right)$ é conexo.

Lema 2.11. ([Fa2], 3.10) Seja K um espaço métrico compacto sem pontos isolados. Dados

(a) Uma sequência $\left(f_{n}: n \in \mathbb{N}\right)$ de funções contínuas, duas a duas disjuntas de $K$ em $[0,1]$;

(b) Uma sequência $\left(x_{n}: n \in \mathbb{N}\right)$ em $K$;

(c) $U m \varepsilon>0$;

(d) Uma sequência limitada $\left(\mu_{n}: n \in \mathbb{N}\right)$ de medidas regulares sobre $K$ tais que $\left|\int f_{n} d \mu_{n}\right|>\varepsilon$, para todo $n \in \mathbb{N}$.

existem $\delta>0, a \subseteq \mathbb{N}$ infinito e funções contínuas $f_{n}^{\prime}: K \longrightarrow[0,1]$ tais que $\operatorname{supp}\left(f_{n}^{\prime}\right) \subseteq \operatorname{supp}\left(f_{n}\right)$ e para todo $b \subseteq$ a temos:

(e) $\left|\int f_{n}^{\prime} d \mu_{n}\right|>\delta$ e $\Sigma\left\{\int f_{m}^{\prime} d\left|\mu_{n}\right|: m \neq n, m \in a\right\}<\delta / 3$, para todo $n \in a$;

(f) $L=K\left(\left(f_{n}^{\prime}\right)_{n \in b}\right)$ é uma extensão completa;

(g) $\Delta\left(\left(f_{n}^{\prime}\right)_{n \in b}\right)$ é unitário ou é disjunto de $\left\{x_{n}: n \in \mathbb{N}\right\}$.

Apesar de todo o esforço para garantir a conexidade da extensão, nenhum dos artigos mencionados prova que a extensão não preserva, em geral, a conexidade. Esse será o objetivo principal da seção 2.4 . 


\subsection{Supremos de álgebras de Boole e extensões por funções contínuas}

Vamos agora mostrar a relação que existe entre adicionar supremos em álgebras de Boole e o método de extensões por funções contínuas em espaços compactos 0-dimensionais. Se tomarmos uma álgebra de Boole $A$, podemos identificar o espaço dos ultrafiltros $S(A)$ com o subespaço fechado de $\{0,1\}^{A}$ formado por todos os homomorfismos de $A$ em $\{0,1\}$. Mais precisamente, temos o seguinte resultado:

Lema 2.12. ([Kop], 7.11) Seja A uma álgebra de Boole. Então o conjunto $\{x: A \rightarrow\{0,1\}$ tal que $x$ é homomorfismo $\}$ é um subespaço fechado de $\{0,1\}^{A}$ homeomorfo a $S(A)$.

Usando a identificação dada pelo lema anterior, temos a seguinte proposição, que nos diz como se relacionam supremos de álgebras de Boole e extensões por funções contínuas.

Proposição 2.13. Seja A uma álgebra de Boole. Para cada a $\in$ A considere $[a]=\{x \in S(A): x(a)=1\}$ o aberto-fechado correspondente ao elemento $a$ de $A$. Sejam também $\left(a_{n}\right)_{n \in \mathbb{N}} \subseteq A$ uma sequência de elementos de $A$ dois a dois disjuntos, s o supremo de tal sequência no completamento de $A$ e $f_{n}: S(A) \longrightarrow[0,1]$ as funções características de $\left[a_{n}\right]$, para todo $n \in \mathbb{N}$. Defina $L_{1}=S(A)\left(\left(f_{n}\right)_{n \in \mathbb{N}}\right)$, a extensão de $S(A)$ pelas funções $\left(f_{n}\right)_{n \in \mathbb{N}}$, e $L_{2}=\{x\lceil A \cup\{s\}: x \in S(B)\}$, onde $B=<A \cup\{s\}>$ é a álgebra de Boole gerada por $A \cup\{s\}$. Então $L_{1}=L_{2}$ e, além disso, existe uma topologia sobre $L_{2}$ induzida por uma bijeção natural entre $S(B)$ e $L_{2}$.

Demonstração. Seja $(x, t) \in L_{1}$ qualquer e mostremos que $t \in\{0,1\}$. Com efeito, suponha $t \neq 0$ e $t \neq 1$. Então existiria aberto $V \times W$ de $S(A) \times[0,1]$ $\operatorname{com}(x, t) \in V \times W$ e $\{0,1\} \cap W=\emptyset$, tal que

$$
(V \times W) \cap G r\left(\Sigma_{n \in \mathbb{N}} f_{n} \mid D\left(\left(f_{n}\right)_{n \in \mathbb{N}}\right)\right) \neq \emptyset .
$$

Portanto, existira também $\left(y, t^{\prime}\right) \in V \times W$ satisfazendo $y \in D\left(\left(f_{n}\right)_{n \in \mathbb{N}}\right)$ e $t^{\prime}=\Sigma_{n \in \mathbb{N}} f_{n}(y)$. Como as funções $f_{n}$ são funções características de $\left[a_{n}\right]$, 
temos que $t^{\prime} \in\{0,1\}$ e, com maior razão, seguiria que $\{0,1\} \cap W \neq \emptyset$, contradição. Concluímos então que $t=0$ ou $t=1$. Vamos agora analisar os três casos possíveis:

1) $(x, 0) \in L_{1}$ e $(x, 1) \notin L_{1}:$ Nesse caso, existe $a \in A$ tal que $x \in[a]$ e $[a] \cap\left[a_{n}\right]=\emptyset$, para todo $n \in \mathbb{N}$. Em outras palavras, $x(a)=1 \mathrm{e}$ $a$ é disjunto de $a_{n}$, para todo $n \in \mathbb{N}$. Note que tal elemento $a \in A$ existe pois, do contrário, se todo $a \in A$ satisfazendo $x \in[a]$ fosse tal que $[a] \cap\left[a_{n}\right] \neq \emptyset$ para algum $n$, teríamos que toda vizinhança de $x$ intersectaria $\operatorname{supp}\left(f_{n}\right)=\left[a_{n}\right]$ para algum $n$. Logo, em toda vizinhança $V$ de $x$ existira $y \in V \cap D\left(\left(f_{n}\right)_{n \in \mathbb{N}}\right)$ tal que $f_{n}(y)=1$, o que implicaria que $(x, 1) \in L_{1}$, contradição.

Logo, para todo $y \in L_{2}$ satisfazendo $y(a)=1$, temos necessariamente $y(s)=0$, já que $a$ e $s$ são disjuntos. Isto nos mostra que $(x, 0) \in L_{2} \mathrm{e}$ $(x, 1) \notin L_{2}$.

2) $(x, 1) \in L_{1}$ e $(x, 0) \notin L_{1}:$ Nesse caso, existe $n \in \mathbb{N}$ tal que $x \in\left[a_{n}\right]$, isto é, tal que $x\left(a_{n}\right)=1$. Isto nos mostra que todo homomorfismo em $S(B)$ que estende $x$ tem que valer 1 em $s$ e, portanto, concluímos que $(x, 1) \in L_{2}$ e $(x, 0) \notin L_{2}$.

3) $(x, 0) \in L_{1}$ e $(x, 1) \in L_{1}:$ Nesse caso, temos que $x\left(a_{n}\right)=0$, para todo $n \in \mathbb{N}$, mas, para todo $a \in A$ temos também que existe $n$ tal que $a \wedge a_{n} \neq \emptyset$. Isso implica que, para todo $a \in A$ tal que $x(a)=1$, temos $a \wedge s \neq 0$ e $a \wedge-s \neq 0$. Precisamos mostrar que existem $y, z \in L_{2}$ tais que ambos estendem $x$ e $y(s)=1$ e $z(s)=0$. Pensemos em $x$ como um ultrafiltro em $A$ sendo que $x(a)=1$ significa que $a \in x$. Temos então que $x \cup\{s\}$ possui a propriedade da intersecção finita e, portanto, existe $y \in S(B)$ tal que $x \cup\{s\} \subseteq y$. Da mesma forma, existe $z \in S(B)$ tal que $x \cup\{-s\} \subseteq z$. Encontramos, portanto, os pontos de $L_{2}$ que queríamos : $y=(x, 1)$ e $z=(x, 0)$.

Mostramos acima que $L_{1} \subseteq L_{2}$. De maneira totalmente análoga mostra-se que $L_{2} \subseteq L_{1}$ e, portanto, concluímos que $L_{1}=L_{2}$. Considere agora a função 
$\varphi: S(B) \longrightarrow L_{2}$ definida por $\varphi(x)=x \uparrow A \cup\{s\}$, para todo $x \in S(B)$. Claramente $\varphi$ é sobrejetora. Além disso, como $A \cup\{s\}$ gera a álgebra $B$, temos que $\varphi$ também é injetora, pois dois homomorfismos que coincidem num conjunto gerador coincidem em toda a álgebra. Por fim, a topologia que colocamos em $L_{2}$ é a topologia induzia por $\varphi$, isto é: $U \subseteq L_{2}$ é aberto se, e somente se, $\varphi^{-1}(U)$ é aberto em $S(B)$.

\subsection{Conexidade e desconexidade das extensões por funções contínuas}

Iniciaremos essa seção mostrando que se $K$ é um espaço topológico compacto localmente conexo e metrizável, então toda extensão de $K$ por funções contínuas é forte, e portanto, conexa.

Teorema 2.14. Seja K um espaço topológico compacto localmente conexo e metrizável. Então toda extensão de $K$ por funções contínuas é forte.

Demonstração. Sejam $K$ como na hipótese do teorema e $\left(f_{n}\right)_{n \in \mathbb{N}} \subseteq C_{1}(K)$ uma sequência de funções duas a duas disjuntas. Tome $L=K\left(\left(f_{n}\right)_{n \in \mathbb{N}}\right)$. Iremos provar que $L$ é uma extensão forte e, para isso, basta mostrarmos que $(x, 0) \in L$, para todo $x \in K \backslash D\left(\left(f_{n}\right)_{n \in \mathbb{N}}\right)$. Como $L$ é compacto e metrizável, mostraremos que $(x, 0) \in L$ construindo uma sequência de pontos $\left(x_{n}, r_{n}\right)$ em $L$ convergindo para $(x, 0)$. Para isso, seja $\left(V_{n}\right)_{n \in \mathbb{N}}$ uma base local decrescente para $x$, onde cada $V_{n}$ é um subconjunto aberto e conexo de $K$. Fixe $n \in \mathbb{N}$. Pela definição 2.2 existe $k_{n} \in \mathbb{N}$ tal que $V_{n} \cap \operatorname{supp}\left(f_{k_{n}}\right) \neq \emptyset$. Logo, existe $y_{n} \in V_{n}$ tal que $f_{k_{n}}\left(y_{n}\right)>0$. Como $V_{n}$ é conexo e $f_{k_{n}}$ é contínua, existe $x_{n} \in V_{n}$ tal que $0<f_{k_{n}}\left(x_{n}\right)<\frac{1}{n}$. Tome $r_{n}=f_{k_{n}}\left(x_{n}\right)$. É fácil ver que $x_{n} \in D\left(\left(f_{n}\right)_{n \in \mathbb{N}}\right)$ e que $\sum_{i \in \mathbb{N}} f_{i}\left(x_{n}\right)=r_{n}$. Portanto, $\left(x_{n}, r_{n}\right) \in L$. Claramente $\left(x_{n}, r_{n}\right) \rightarrow(x, 0)$ e isso encerra a prova do teorema.

O próximo resultado nos diz que extensões por funções contínuas nem sempre preservam conexidade: 
Teorema 2.15. Existem um continuum $K$ e uma sequência de funções duas a duas disjuntas $\left(f_{n}\right)_{n \in \mathbb{N}} \subseteq C_{1}(K)$ tais que $K\left(\left(f_{n}\right)_{n \in \mathbb{N}}\right)$ é desconexo.

Demonstração. Para cada $n \in \mathbb{N}$, defina $g_{n}:[0,1] \longrightarrow[0,1]$ como

$$
g_{n}(x)= \begin{cases}\operatorname{sen}\left(\pi\left(2^{n+1} x-1\right)\right), & \text { se } x \in\left[\frac{1}{2^{n+1}}, \frac{1}{2^{n}}\right] \\ 0, & \text { caso contrário }\end{cases}
$$

Observe que $g_{n}\left(\frac{1}{2^{n+1}}\right)=g_{n}\left(\frac{1}{2^{n}}\right)=0$ e $g_{n}\left(\frac{3}{2^{n+2}}\right)=1$. Além disso, $g_{n}$ é estritamente crescente em $\left[\frac{1}{2^{n+1}}, \frac{3}{2^{n+2}}\right]$ e estritamente decrescente em $\left[\frac{3}{2^{n+2}}, \frac{1}{2^{n}}\right]$.

Para simplificar a notação, chamaremos $a_{n}=\frac{1}{2^{n+1}}$ e $b_{n}=\frac{3}{2^{n+2}}$, para todo $n \in \mathbb{N}$.

Seja $K_{0}$ a extensão de $[0,1]$ por $\left(g_{n}\right)_{n \in \mathbb{N}}$. Defina $K=K_{0} \cup(\{0\} \times[1,2])$ e, para cada $n \in \mathbb{N}$, considere a função $f_{n}: K \longrightarrow[0,1]$ dada por

$$
f_{n}(x, t)= \begin{cases}t, & \text { se } x \in\left(a_{n+1}, a_{n}\right) \\ 0, & \text { caso contrário }\end{cases}
$$

Pelo Teorema 2.14, $K_{0}$ é conexo. Como $K_{0}$ é fechado em $K \times[0,1]$ e $\left(\left(b_{n}, 1\right)\right)_{n \in \mathbb{N}}$ é uma sequência em $K_{0}$ convergindo para $(0,1)$, temos que $(0,1) \in K_{0} \cap(\{0\} \times[1,2])$. Como ambos os conjuntos são conexos, concluímos que $K$ é conexo.

Usando a continuidade de $g_{n}$, é fácil verificar que $f_{n}$ é contínua. A sequência $\left(f_{n}\right)_{n \in \mathbb{N}}$ é claramente formada por funções duas a duas disjuntas.

Seja $L=K\left(\left(f_{n}\right)_{n \in \mathbb{N}}\right)$. Observe que

$$
\{0\} \times(1,2] \subset D\left(\left(f_{n}\right)_{n \in \mathbb{N}}\right),
$$

pois $\{0\} \times(1,2]$ é um aberto de $K$ que não intersecta nenhum dos suportes das $f_{n}$ ' s. Logo, $\{0\} \times(1,2] \times\{0\} \subset L$ e, como $L$ é fechado, temos que $\{0\} \times[1,2] \times\{0\} \subset L$.

Claramente, $\{0\} \times[1,2] \times\{0\}$ é fechado em $L$. Para concluir o teorema é suficiente provar que esse conjunto é aberto em $L$.

Seja $\left(z_{n}\right)_{n \in \mathbb{N}}$ uma sequência em $L \backslash\{0\} \times[1,2] \times\{0\}$ convergindo para $z \in L$. Precisamos provar que $z \notin\{0\} \times[1,2] \times\{0\}$. 
Podemos assumir que $z_{n} \in G r\left(\Sigma_{n \in \mathbb{N}} f_{n} \mid D\left(\left(f_{n}\right)_{n \in \mathbb{N}}\right)\right)$, pois o gráfico é denso em $L$.

Note que, se $(0, t) \in K$, para $0<t \leq 1$, toda vizinhança aberta de $(0, t)$ intersecta $\operatorname{supp}\left(f_{n}\right)$ para infinitos valores de $n$. Isso segue do fato de que cada $g_{n}$ é sobrejetora em $[0,1]$, e os suportes das $g_{n}$ convergem para 0.

Logo, temos que $(0, t) \notin D\left(\left(f_{n}\right)_{n \in \mathbb{N}}\right)$, para $0<t \leq 1$. Portanto, segue que $z_{n} \notin\{0\} \times(0,1] \times[0,1]$.

Sejam $z_{n}=\left(x_{n}, y_{n}, t_{n}\right)$ e $z=\left(x^{\prime}, y^{\prime}, t^{\prime}\right)$. Suponha que $z \in\{0\} \times[1,2] \times\{0\}$. Isto é, $z=\left(0, y^{\prime}, 0\right)$, onde $1 \leq y^{\prime} \leq 2$. Passando a uma subsequência, podemos assumir que $y_{n}>\frac{1}{2}$, para todo $n$. Pela observação acima, podemos também assumir que $x_{n}>0$, para todo $n$. Mas é fácil verificar que isso implica que

$$
x_{n} \in \operatorname{supp}\left(g_{k_{n}}\right) \text {, }
$$

para algum $k_{n} \in \mathbb{N}$. Logo, para todo $n \in \mathbb{N}$,

$$
\left(x_{n}, y_{n}\right) \in \operatorname{supp}\left(f_{k_{n}}\right) .
$$

Portanto, $t_{n}=y_{n}$, para todo $n$. Mas $y_{n}>\frac{1}{2}$, contradizendo que $\left(x_{n}, y_{n}, t_{n}\right)$ converge para $\left(0, y^{\prime}, 0\right)$.

Mostramos que $\{0\} \times[1,2] \times\{0\}$ é um aberto-fechado de $L$, o que prova que $L$ é desconexo.

O próximo teorema mostra que podemos perder a conexidade de $[0,1]$ por uma dupla extensão.

Teorema 2.16. Existem um continuum $K$ e uma sequência de funções duas a duas disjuntas $\left(f_{n}\right)_{n \in \mathbb{N}} \subseteq C_{1}(K)$ tais que $K$ é uma extensão de [0,1] por funções contínuas e $K\left(\left(f_{n}\right)_{n \in \mathbb{N}}\right)$ é desconexo.

Demonstração. Sem perda de generalidade, trocaremos o intervalo $[0,1]$ pelo intervalo $[-1,1]$, para manter a notação do teorema 2.15. Sejam $g_{n}, a_{n}$, $b_{n}$ como na prova do teorema 2.15 , estendendo $g_{n}$ para $[-1,1]$ através da definição $g_{n}(x)=0$, para $x \leq 0$. Defina $K$ a extensão de $[-1,1]$ por $\left(g_{n}\right)_{n \in \mathbb{N}}$ 
e $f_{n}: K \longrightarrow[0,1]$, para $n \in \mathbb{N}$, como

$$
f_{n}(x, t)= \begin{cases}1-t, & \text { se } x \in\left[b_{n}, b_{n+1}\right] \\ 0, & \text { caso contrário. }\end{cases}
$$

Para cada $n \in \mathbb{N}$ provaremos a continuidade de $f_{n}$. Seja $\left(x_{i}, t_{i}\right)_{i \in \mathbb{N}}$ uma sequência convergindo para $(x, t)$ em $K$. Precisamos mostrar que $f_{n}\left(x_{i}, t_{i}\right)$ converge para $f_{n}(x, t)$.

Se $x \in\left[b_{n}, a_{n+1}\right)$, temos que $x \in \operatorname{supp}\left(g_{n}\right) \cap D\left(\left(g_{n}\right)_{n \in \mathbb{N}}\right)$ e, portanto, $t=g_{n}(x)$. Como $t \neq 0$, podemos assumir que $t_{i}=g_{n}\left(x_{i}\right)$. Pela continuidade de $g_{n}$ temos $g_{n}\left(x_{i}\right)$ convergindo para $g_{n}(x)$ e, portanto, $f_{n}\left(x_{i}, t_{i}\right)=1-g_{n}\left(x_{i}\right)$ convergindo para $f_{n}(x, t)=1-g_{n}(x)$.

Analogamente, se $x \in\left(a_{n+1}, b_{n+1}\right]$ temos que $t=g_{n+1}(x)$ e $f_{n}\left(x_{i}, t_{i}\right)$ converge para $f_{n}(x, t)$.

Se $x=a_{n+1}$, tanto $g_{n}\left(x_{i}\right)$ como $g_{n+1}\left(x_{i}\right)$ convergem para 0 , para $i \in \mathbb{N}$. Então $f_{n}\left(x_{i}, t_{i}\right)$ converge para $1=f_{n}(x, t)$.

Se $x \leq b_{n}$ ou $x \geq b_{n+1}$ é fácil verificar que $f_{n}\left(x_{i}, t_{i}\right)$ converge para 0 , que é igual a $f_{n}(x, t)$.

Seja $L$ a extensão de $K$ por $\left(f_{n}\right)_{n \in \mathbb{N}}$. Vamos provar que $L$ é desconexo. Para isso, precisamos mostrar que $[-1,0] \times\{0\} \times\{0\}$ - que é claramente um subconjunto fechado de $L$ - é aberto em $L$.

A prova é análoga a do Teorema 2.15. Seja $z_{n}=\left(x_{n}, y_{n}, t_{n}\right)$ uma sequência em $L \backslash[-1,0] \times\{0\} \times\{0\}$ convergindo para $z=(x, y, t) \in L$. Precisamos provar que $z \notin L$.

Podemos assumir que $z_{n} \in G r\left(\Sigma_{n \in \mathbb{N}} f_{n} \mid D\left(\left(f_{n}\right)_{n \in \mathbb{N}}\right)\right)$, pois o gráfico é denso em $L$. Em particular, $\left(x_{n}, y_{n}\right) \in D\left(\left(f_{n}\right)_{n \in \mathbb{N}}\right)$.

Observe que $\{0\} \times(0,1)$ está contido em $K$, mas é disjunto de $D\left(\left(f_{n}\right)_{n \in \mathbb{N}}\right)$. Logo podemos assumir que $x_{n} \neq 0$, para todo $n$. Além disso, note que

$$
\pi_{L,[-1,1]}^{-1}([-1,0))=[-1,0) \times\{0\} \times\{0\}
$$

pois $[-1,0) \subset D\left(\left(g_{n}\right)_{n \in \mathbb{N}}\right)$ e $[-1,0) \times\{0\} \subset D\left(\left(f_{n}\right)_{n \in \mathbb{N}}\right)$. Logo, também podemos assumir que $x_{n}>0$, para todo $n$.

Suponha $z \in[-1,0] \times\{0\} \times\{0\}$. Em particular, $z=(x, 0,0)$, para algum 
$x$. Podemos assumir que $y_{n}<\frac{1}{2}$, para todo $n$. Como $x_{n}>0$, existe $i$ tal que $x_{n} \in\left[b_{i}, b_{i+1}\right]$. Logo,

$$
t_{n}=f_{i}\left(x_{n}, y_{n}\right)=1-y_{n}>\frac{1}{2}
$$

o que contradiz que $t_{n}$ converge para 0 .

Antes de enunciarmos o principal teorema deste capítulo, necessitamos do seguinte lema:

Lema 2.17. Sejam $K$ um espaço topológico compacto e $\left(f_{n}\right)_{n \in \mathbb{N}} \subseteq C_{1}(K)$ uma sequência de funções duas a duas disjuntas. Suponha que existe um fechado $L \subseteq K$ tal que $\operatorname{supp}\left(f_{n}\right) \subset L$, para todo $n \in \mathbb{N}$. Então,

$$
K\left(\left(f_{n}\right)_{n \in \mathbb{N}}\right)=L\left(\left(f_{n} \mid L\right)_{n \in \mathbb{N}}\right) \bigcup(\overline{K \backslash L}) \times\{0\}
$$

Demonstração. Denotaremos por $D$ o conjunto $D\left(\left(f_{n}\right)_{n \in \mathbb{N}}\right)$. Notemos primeiramente que $K \backslash L$ é um conjunto aberto de $K$ onde $f_{n}$ é nula para todo $n$. Temos então que $K \backslash L \subset D$. Como $\operatorname{supp}\left(f_{n}\right) \subset L$, temos que $V \cap \operatorname{supp}\left(f_{n}\right) \neq \emptyset$ se e somente se $(V \cap L) \cap \operatorname{supp}\left(f_{n} \mid L\right) \neq \emptyset$, sempre que $n \in \mathbb{N}$ e $V$ é aberto em $K$. Logo,

$$
D\left(\left(f_{n} \mid L\right)_{n \in \mathbb{N}}\right)=D \cap L
$$

Concluímos então que

$$
L\left(\left(f_{n} \mid L\right)_{n \in \mathbb{N}}\right)=\overline{G r\left(\sum_{n \in \mathbb{N}} f_{n} \mid D \cap L\right)} .
$$

Isso é suficiente para provar o lema, já que temos as seguintes igualdades:

$$
K\left(\left(f_{n}\right)_{n \in \mathbb{N}}\right)=\overline{G r\left(\Sigma_{n \in \mathbb{N}} f_{n} \mid D\right)}=\overline{G r\left(\Sigma_{n \in \mathbb{N}} f_{n} \mid D \cap L\right) \cup(K \backslash L) \times\{0\}} .
$$

O teorema principal desse capítulo nos mostra que partindo de qualquer espaço topológico compacto conexo e metrizável, podemos obter um espaço 
desconexo depois de três extensões sucessivas.

Teorema 2.18. Seja $K_{0}$ um continuum infinito qualquer. Então existem compactos $K_{1}, K_{2}$ e $K_{3}$ tais que $K_{i}$ é uma extensão de $K_{i-1}$ por funções contínuas, para $1 \leq i \leq 3$, e $K_{3}$ é desconexo.

Demonstração. Usando a metrizabilidade de $K_{0}$, fixe $\left(\bar{x}_{n}\right)_{n \in \mathbb{N}}$ uma sequência convergente em $K_{0}$ e seja $\bar{x}$ o seu limite. Seja $\left(U_{n}\right)_{n \in \mathbb{N}}$ uma sequência de abertos dois a dois disjuntos tal que $\bar{x}_{n} \in U_{n}$ e $\operatorname{diam}\left(U_{n}\right) \leq \frac{1}{n}$ (i.e., para uma métrica fixada $d$ sobre $K_{0}$, temos $d(x, y) \leq \frac{1}{n}$, para todo $\left.x, y \in U_{n}\right)$. Usando o Lema de Urysohn e a normalidade, encontramos, para cada $n$, uma função $e_{n}: K_{0} \longrightarrow[0,1]$ cujo suporte está contido em $U_{n}$ e tal que $e_{n}\left(\bar{x}_{n}\right)=1$. Pela conexidade de $K_{0}, e_{n}\left[K_{0}\right]=[0,1]$. Defina $K_{1}=K_{0}\left(\left(e_{n}\right)_{n \in \mathbb{N}}\right)$.

Afirmação 2.18.1. Podemos assumir que $e_{n}^{-1}\left[\left\{\frac{1}{2}\right\}\right]$ é raro em $K_{0}$.

Como espaços compactos metrizáveis satisfazem a condição de cadeia enumerável, existe $r \in[0,1]$ tal que $e_{n}^{-1}[\{r\}]$ é raro. Caso contrário, teríamos uma quantidade não enumerável de abertos dois a dois disjuntos em $K_{0}$. Tome $h:[0,1] \longrightarrow[0,1]$ uma função contínua, bijetora e crescente tal que $h(r)=\frac{1}{2}$. Troque $e_{n}$ por $h \circ e_{n}$. Repita o processo para todo $n \in \mathbb{N}$.

Afirmação 2.18.2. $D\left(\left(e_{n}\right)_{n \in \mathbb{N}}\right)=K_{0} \backslash\{\bar{x}\}$.

Para provarmos a afirmação notemos que para toda vizinhança aberta $U$ de $\bar{x}$, existe um conjunto finito $F \subset \mathbb{N}$ tal que $U_{n} \subset U$, para todo $n \in \mathbb{N} \backslash F$. Como $\operatorname{supp}\left(e_{n}\right) \subset U_{n}$ e é não vazio, temos que $\bar{x} \in K_{0} \backslash D\left(\left(e_{n}\right)_{n \in \mathbb{N}}\right)$. Por outro lado, se $x \neq \bar{x}$, por Hausdorff existem abertos disjuntos $V$ e $U$ tais que $x \in V$ e $\bar{x} \in U$. Temos que $U_{n} \subset U$ para todos, exceto uma quantidade finita de $n \in \mathbb{N}$, o que nos permite concluir que $x \in D\left(\left(e_{n}\right)_{n \in \mathbb{N}}\right)$ e isso encerra a prova da afirmação.

Afirmação 2.18.3. $\pi_{K_{1}, K_{0}}^{-1}[\bar{x}]=\{\bar{x}\} \times[0,1]$.

Seja $t \in[0,1]$. Usando que toda $e_{n}$ é sobrejetora em $[0,1]$, tome $y_{n} \in U_{n}$ tal que $e_{n}\left(y_{n}\right)=t$. Claramente, $\left(y_{n}\right)_{n \in \mathbb{N}}$ converge para $\bar{x}$ e todo par $\left(y_{n}, t_{n}\right)$ pertence ao gráfico de $\Sigma_{n \in \mathbb{N}} e_{n} \mid D\left(\left(e_{n}\right)_{n \in \mathbb{N}}\right)$, que está contido em $K_{1}$. Logo, pela compacidade de $K_{1}$, temos que $(\bar{x}, t) \in K_{1}$, o que prova a afirmação. 
Sejam $\left(a_{n}\right)_{n \in \mathbb{N}}$ e $\left(b_{n}\right)_{n \in \mathbb{N}}$ sequências em $[0,1]$ ambas convergindo para $\frac{1}{2}$ tais que $a_{n+1}<b_{n}<a_{n}<1$, para todo $n \in \mathbb{N}$. Defina $\tilde{f}_{n}:[0,1] \longrightarrow[0,1]$ com suporte contido em $\left[a_{n+1}, a_{n}\right]$ e tal que $\tilde{f}_{n}\left(b_{n}\right)=1$. Suponha também que $\tilde{f}_{n}$ é monótona em cada intervalo $\left[a_{n+1}, b_{n}\right]$ e $\left[b_{n}, a_{n}\right]$, como na definição de $g_{n}$ na prova do Teorema 2.15.

Tome $f_{n}(x, t)=\tilde{f}_{n}(t)$, para todo $(x, t) \in K_{1} \subset K_{0} \times[0,1]$. Defina $K_{2}=K_{1}\left(\left(f_{n}\right)_{n \in \mathbb{N}}\right)$.

Afirmação 2.18.4. $K_{1} \backslash\left(K_{0} \times\left\{\frac{1}{2}\right\}\right) \subset D\left(\left(f_{n}\right)_{n \in \mathbb{N}}\right)$.

A afirmação segue do fato de que $D\left(\left(\tilde{f}_{n}\right)_{n \in \mathbb{N}}\right)=[0,1] \backslash\left\{\frac{1}{2}\right\}$. Se tomarmos $t \neq \frac{1}{2}$ e $V$ uma vizinhança aberta de $t$ que intersecta $\operatorname{supp}\left(\tilde{f}_{n}\right)$ para finitos $n$ 's, o conjunto $\left(K_{0} \times V\right) \cap K_{1}$ intersecta $\operatorname{supp}\left(f_{n}\right)$ para finitos $n$ 's, já que $f_{n}(x, t)=\tilde{f}_{n}(t)$.

Seja $K^{\prime}$ a extensão de $[0,1]$ por $\left(\tilde{f}_{n}\right)_{n \in \mathbb{N}}$. Seja $\tilde{g}_{n}: K^{\prime} \longrightarrow[0,1]$ definida como a $f_{n}$ na prova do teorema 2.16. Temos então $K^{\prime}\left(\left(\tilde{g}_{n}\right)_{n \in \mathbb{N}}\right)$ desconexo. Denote essa extensão por $K^{\prime \prime}$. Defina

$$
g_{n}(x, y, z)=\tilde{g}_{n}(y, z)
$$

sobre $K_{2}$.

Afirmação 2.18.5. Toda $g_{n}$ é bem definida e contínua, e $\left(g_{n}\right)_{n \in \mathbb{N}}$ é uma sequência de funções duas a duas disjuntas.

A continuidade e o fato de serem funções duas a duas disjuntas segue imediatamente da definição, já que a projeção é contínua. Para provar que as funções estão bem definidas, precisamos mostrar que $(y, z) \in K^{\prime}$, sempre que $(x, y, z) \in K_{2}$. Se $y \neq \frac{1}{2}$, segue da afirmação 2.18 .4 que $z=f_{n}(x, y)$ e, portanto, $z=\tilde{f}_{n}(y)$. Temos então que $(y, z) \in K^{\prime}$. Se $y=\frac{1}{2}$, pela construção temos que $(y, z) \in K^{\prime}$ para todo $z \in[0,1]$, provando a afirmação. Tomamos agora $K_{3}=K_{2}\left(\left(g_{n}\right)_{n \in \mathbb{N}}\right)$.

Afirmação 2.18.6. $K_{3} \cap\left(K_{0} \times\left\{\frac{1}{2}\right\} \times[0,1]^{2}\right)$ é raro em $K_{3} \cap\left(K_{0} \times\left[\frac{1}{2}, 1\right] \times\right.$ $\left.[0,1]^{2}\right)$. 
Pela afirmação 2.18.1, $K_{1} \cap\left(K_{0} \times\left\{\frac{1}{2}\right\}\right)$ é raro em $K_{1}$. Usando o lema 2.17, $K_{2} \cap\left(K_{0} \times\left[\frac{1}{2}, 1\right] \times[0,1]\right)$ é a extensão de $K_{1} \cap\left(K_{0} \times\left[\frac{1}{2}, 1\right]\right)$ pelas restrições de $\left(f_{n}\right)_{n \in \mathbb{N}}$, lembrando que $f_{n}(x, t)=0$, se $t<\frac{1}{2}$. Portanto, como extensões preservam conjuntos raros na projeção inversa (veja [Ko2]), concluímos que $K_{2} \cap\left(K_{0} \times\left\{\frac{1}{2}\right\} \times[0,1]\right)$ é raro em $K_{2} \cap\left(K_{0} \times\left[\frac{1}{2}, 1\right] \times[0,1]\right)$. Basta agora repetir os mesmos argumentos na próxima extensão, e com isso encerramos a prova da afirmação.

Seja $L=K_{3} \cap\left(K_{0} \times\left[0, \frac{1}{2}\right] \times\{(0,0)\}\right)$. Claramente $L$ é um subespaço fechado de $K_{3}$. Para concluir a desconexidade de $K_{3}$, é suficiente provar a seguinte afirmação:

Afirmação 2.18.7. $\overline{K_{3} \backslash L} \cap L=\emptyset$.

Por contradição, suponha que existe uma sequência $\left(x_{n}, t_{n}, s_{n}, r_{n}\right)$ em $K_{3} \backslash L$ convergindo para $(x, t, s, r) \in L$. Pela metrizabilidade e utilizando a afirmação 2.18.6 podemos assumir que $t_{n}>\frac{1}{2}$, para todo $n$. Portanto, como provamos, $r_{n}=\tilde{g}_{m}\left(t_{n}, s_{n}\right)$, para algum $m$. Então temos $\left(t_{n}, s_{n}, r_{n}\right) \in K^{\prime \prime}$. Claramente temos $(t, s, r)=\left(\frac{1}{2}, 0,0\right)$. Isso contradiz o argumento final na prova do teorema 2.16, quando provamos a desconexidade de $K^{\prime \prime}$. 


\section{Capítulo 3}

\section{Espaços de Banach da forma $C(K)$ essencialmente incomparáveis}

Dizemos que dois espaços de Banach são totalmente incomparáveis se nenhum subespaço de dimensão infinita de um espaço for isomorfo a um subespaço do outro. Em [Ga], Gasparis construiu uma família de tamanho $2^{\omega}$ de espaços de Banach indecomponíveis, separáveis e dois a dois totalmente incomparáveis. Como os espaços são separáveis, $2^{\omega}$ é o maior tamanho possível para uma tal família. Sabemos que $C(K)$ de dimensão infinita possui uma cópia de $c_{0}$ como subespaço e, portanto, espaços de Banach da forma $C(K)$ não podem ser totalmente incomparáveis. Utilizaremos neste capítulo uma noção mais fraca de incomparabilidade, devida a Aiena e González (veja [AG]), chamada incomparabilidade essencial, apresentada na Definição 3.12. Em [Fa3], Fajardo constrói, assumindo $\diamond$, uma família $\left(C\left(K_{\xi}\right)\right)_{\xi<2^{\left(2^{\omega}\right)}}$ de espaços de Banach indecomponíveis e dois a dois essencialmente incomparáveis. No exemplo construído por Fajardo, cada $K_{\xi}$ era um espaço de Koszmider. Unindo as técnicas presentes em [Fa2] e [Fa3] construíremos, assumindo $\diamond$, uma família $\left(K_{\xi}\right)_{\xi<2^{\left(2^{\omega}\right)}}$ de espaços conexos e hereditariamente Koszmider tais que $C\left(K_{\xi}\right)$ e $C\left(K_{\eta}\right)$ são essencialmente incomparáveis, para todo $\xi \neq \eta$. Como espaços hereditariamente Koszmider não contêm cópia de $\beta \mathbb{N}$ e nem sequências con- 
vergentes não trivias, cada espaço $K_{\xi}$ responde positivamente ao Problema de Efimov, sobre a existência de um compacto que não possui sequências convergentes não triviais nem $\beta \mathbb{N}$ como subespaço. O problema de Efimov já havia sido respondido positivamente em 1975 por Fedorchuk (veja [Fed]) assumindo apenas CH. O problema continua em aberto em ZFC, e tão pouco sabemos se é consistente com ZFC a negação do problema de Efimov.

\subsection{O Axioma}

Apresentaremos nesta seção a definição e também alguns resultados básicos envolvendo o axioma $\diamond$ (lê-se diamante). O axioma $\diamond$ é relativamente consistente com ZFC, valendo no universo construtível de Gödel. Além disso, como veremos a seguir, o axioma $\diamond$ implica $\mathrm{CH}$. Para maiores referências indicamos $[\mathrm{Ku}]$, [Je] e [Ve].

Definição 3.1. Dizemos que um subconjunto $C$ de $\omega_{1}$ é fechado ilimitado se é ilimitado e $\sup B \in C$, para todo $B \subseteq C$ enumerável. Dizemos que um subconjunto $S$ de $\omega_{1}$ é estacionário se intersecta todo fechado ilimitado.

Note que subconjuntos estacionários de $\omega_{1}$ são ilimitados pois o conjunto $\left\{\alpha<\omega_{1}: \alpha>\beta\right\}$ é fechado ilimitado para cada $\beta<\omega_{1}$.

O próximo lema é um resultado bastante conhecido, que pode ser encontrado em [Ku, Capítulo II, Lema 6.8].

Lema 3.2. A intersecção de uma família enumerável de fechados ilimitados de $\omega_{1}$ é um conjunto fechado ilimitado. Em particular, se $S$ é estacionário e $C$ é fechado ilimitado, então $S \cap C$ é estacionário.

Axioma $\diamond$. Existe uma sequência $\left(X_{\alpha}\right)_{\alpha \in \omega_{1}}$ tal que $X_{\alpha} \subseteq \alpha$ para todo $\alpha \in \omega_{1}$, e o conjunto $\left\{\alpha \in \omega_{1}: X \cap \alpha=X_{\alpha}\right\}$ é estacionário para todo $X \subseteq \omega_{1}$.

Notação. A sequência $\left(X_{\alpha}\right)_{\alpha \in \omega_{1}}$ é chamada $\diamond$-sequência. 
Não é difícil ver que o axioma $\diamond$ implica $\mathrm{CH}$, conforme demonstrado no lema abaixo.

Lema 3.3. $\diamond \rightarrow C H$.

Demonstração. Seja $X \subseteq \omega$ um subconjunto qualquer. Como conjuntos estacionários são ilimitados, existe $\alpha>\omega$ tal que $X=X \cap \alpha=X_{\alpha}$. Logo $\left(X_{\alpha}\right)_{\alpha<\omega_{1}}$ contém todos os subconjuntos de $\omega$ e isso encerra a demonstração do lema.

O Próximo lema nos será útil ao longo do capítulo. Usaremos ${ }^{\alpha} 2$ para denotar o conjunto das funções de $\alpha$ em $2=\{0,1\}$ e ${ }^{\omega_{1}} 2$ para denotar o conjunto das funções de $\omega_{1}$ em $2=\{0,1\}$.

Lema 3.4. O axioma $\diamond$ implica:

(a) Se $\left(B_{\alpha}\right)_{\alpha<\omega_{1}}$ é uma sequência de conjuntos de tamanho $\omega_{1}$, existe uma sequência $\left\{x_{\alpha}: \alpha<\omega_{1}\right\}$ tal que $x_{\alpha} \in \Pi_{\beta<\alpha} B_{\beta}$ e, para todo elemento $x \in \Pi_{\alpha<\omega_{1}} B_{\alpha}$ o conjunto $\left\{\alpha<\omega_{1}: x \mid \alpha=x_{\alpha}\right\}$ é estacionário;

(b) Se $\left(X_{\alpha}\right)_{\alpha<\omega_{1}}$ é uma sequência de conjuntos de tamanho $\omega_{1}$, existe uma sequência $\left(t_{\alpha}, s_{\alpha}, x_{\alpha}\right)_{\alpha<\omega_{1}}$ tal que $t_{\alpha}, s_{\alpha} \in{ }^{\alpha} 2, x_{\alpha} \in \Pi_{\beta<\alpha} X_{\beta}$ e para todo $t, s \in{ }^{\omega_{1}} 2$ e $x \in \Pi_{\alpha<\omega_{1}} X_{\alpha}$ o conjunto

$$
\left\{\alpha<\omega_{1}: t\left|\alpha=t_{\alpha}, s\right| \alpha=s_{\alpha} \text { e } x \mid \alpha=x_{\alpha}\right\}
$$

é estacionário.

Demonstração. Para provarmos $(a)$ seja $\left(X_{\alpha}\right)_{\alpha<\omega_{1}}$ uma $\diamond$-sequência. Seja também $\left\{\xi_{\alpha}: \alpha<\omega_{1}\right\}$ uma sequência crescente em $\omega_{1}$ definida da seguinte maneira: $\xi_{\alpha+1}=\xi_{\alpha}+\omega$ e $\xi_{\alpha}=\sup \left\{\xi_{\alpha^{\prime}}: \alpha^{\prime}<\alpha\right\}$ para $\alpha$ limite.

Para cada $\alpha<\omega_{1}$ seja $\phi_{\alpha}: \mathcal{P}\left(\left[\xi_{\alpha}, \xi_{\alpha+1}\right)\right) \rightarrow B_{\alpha}$ uma função bijetora, que existe pois $\diamond \rightarrow \mathrm{CH}$. Definimos então $x_{\alpha} \in \Pi_{\beta<\alpha} B_{\beta}$ dado por

$$
x_{\alpha}(\beta)=\phi_{\beta}\left(X_{\xi_{\alpha}} \cap\left[\xi_{\beta}, \xi_{\beta+1}\right]\right),
$$

para todo $\beta<\omega_{1}$. 
Mostraremos que a sequência $\left(x_{\alpha}\right)_{\alpha<\omega_{1}}$ satisfaz $(a)$. Seja $x \in \Pi_{\alpha<\omega_{1}} B_{\alpha}$. Seja $X=\cup\left\{\phi_{\alpha}^{-1}(x(\alpha)): \alpha<\omega_{1}\right\}$. Temos $x \mid \alpha=x_{\alpha}$ se, e somente se, $X \cap \xi_{\alpha}=X_{\xi_{\alpha}}$.

Pelo Lema 3.2 temos que o conjunto

$$
\left\{\alpha<\omega_{1}: X \cap \xi_{\alpha}=X_{\xi_{\alpha}}\right\}=\left\{\beta<\omega_{1}: X \cap \beta=X_{\beta}\right\} \cap\left\{\xi_{\alpha}: \alpha<\omega_{1}\right\}
$$

é estacionário. Mas

$$
\left\{\alpha<\omega_{1}: X \cap \xi_{\alpha}=X_{\xi_{\alpha}}\right\}=\left\{\alpha<\omega_{1}: x \mid \alpha=x_{\alpha}\right\}
$$

concluindo a prova do item $(a)$.

Vamos agora provar o item (b). Para cada $\alpha<\omega_{1}$ defina $B_{\alpha}=2 \times 2 \times X_{\alpha}$. Aplicando o item $(a)$ para $\left(B_{\alpha}\right)_{\alpha<\omega_{1}}$, obtemos uma sequência $\left\{y_{\alpha}: \alpha<\omega_{1}\right\}$ tal que $y_{\alpha} \in \Pi_{\beta<\alpha} B_{\beta}$ e, para todo $y \in \Pi_{\alpha<\omega_{1}} B_{\alpha}$, o conjunto

$$
\left\{\alpha<\omega_{1}: y \mid \alpha=y_{\alpha}\right\}
$$

é estacionário. O resultado segue da identificação de $y_{\alpha} \operatorname{com}\left(t_{\alpha}, s_{\alpha}, x_{\alpha}\right)$.

O lema a seguir será utilizado durante a construção indutiva dos espaços $K_{\xi}$ para garantir certas condições topológicas. Este mesmo lema foi também utilizado por Fajardo, em [Fa2].

Lema 3.5. Seja $Y \subseteq[0,1]^{\omega_{1}}$ e seja $\left(x_{\alpha}\right)_{\alpha<\omega_{1}}$ uma sequência densa em $Y$. Então $\left\{\alpha<\omega_{1}:\left(x_{\beta} \mid \alpha\right)_{\beta<\alpha}\right.$ é denso em $\left.\pi_{\alpha}[Y]\right\}$ é fechado ilimitado em $\omega_{1}$.

Demonstração. Vamos primeiro mostrar que o conjunto a seguir definido por $C=\left\{\alpha<\omega_{1}:\left(x_{\beta} \mid \alpha\right)_{\beta<\alpha}\right.$ é denso em $\left.\pi_{\alpha}[Y]\right\}$ é fechado em $\omega_{1}$, i.e., para toda sequência enumerável crescente em $C$ o supremo de tal sequência continua em $C$. Seja $\left(\alpha_{n}\right)_{n \in \mathbb{N}}$ uma sequência crescente em $C$ e tome $\alpha$ o supremo de $\left(\alpha_{n}\right)_{n \in \mathbb{N}}$ em $\omega_{1}$. Vamos provar que $\alpha \in C$, o que significa dizer que $\left(x_{\beta} \mid \alpha\right)_{\beta<\alpha}$ é denso em $\pi_{\alpha}[Y]$.

Seja $U$ um aberto elementar de $[0,1]^{\alpha}$ que intersecta $\pi_{\alpha}[Y]$. Como $U$ depende de um número finito de coordenadas, existe $n \in \mathbb{N}$ tal que $\alpha_{n}$ contém todas essas coordenadas. Temos então que $U=\pi_{\alpha_{n}}^{-1}\left[\pi_{\alpha_{n}}[U]\right]$ e $\pi_{\alpha_{n}}[U]$ é aberto 
em $[0,1]^{\alpha_{n}}$. Logo, como $\alpha_{n} \in C$, existe $\beta<\alpha$ tal que $x_{\beta} \mid \alpha_{n} \in \pi_{\alpha_{n}}[U]$ e portanto $x_{\beta} \mid \alpha \in U$.

Vamos agora provar que $C$ é ilimitado em $\omega_{1}$. Para isso, seja $\alpha_{0}$ um ordinal em $\omega_{1}$. Pela continuidade da projeção, $\left(x_{\beta} \mid \alpha_{0}\right)_{\beta<\omega_{1}}$ é denso em $\pi_{\alpha_{0}}[Y]$. Como $\pi_{\alpha_{0}}[Y]$ tem peso enumerável, para cada vizinhança aberta de uma base enumerável de $\pi_{\alpha_{0}}[Y]$ tomamos algum $x_{\beta} \mid \alpha_{0}$ pertencente a ela. Assim obtemos $\alpha_{1}<\omega_{1}$, que podemos supor maior que $\alpha_{0}$, tal que $\left(x_{\beta} \mid \alpha_{0}\right)_{\beta<\alpha_{1}}$ é denso em $\pi_{\alpha_{0}}[Y]$. Iterando esse processo, encontramos uma sequência crescente $\left(\alpha_{n}\right)_{n \in \omega_{1}}$ tal que $\left(x_{\beta} \mid \alpha_{n}\right)_{\beta<\alpha_{n+1}}$ é denso em $\pi_{\alpha_{n}}[Y]$. Tomando $\alpha$ o supremo de $\left(\alpha_{n}\right)_{n \in \mathbb{N}}$ e repetindo os argumentos anteriores, concluímos que $\left(x_{\beta} \mid \alpha\right)_{\beta<\alpha}$ é denso em $\pi_{\alpha}[Y]$, provando que $\alpha \in C$.

Iremos reduzir o problema de construir espaços de Banach essencialmente incomparáveis a um problema puramente combinatório. Para isso, introduziremos um lema combinatório, que é uma variação do princípio $A\left(2^{\left(2^{\omega}\right)}\right)$ utilizado por Fajardo, em [Fa3]. Na demonstração do lema identificaremos $2^{\omega_{1}}$ com o conjunto ${ }^{\omega_{1}} 2$ das funções de $\omega_{1}$ em $2=\{0,1\}$.

Lema 3.6. $(\diamond)$ Seja $\kappa=2^{\omega_{1}}$. Se $\left(X_{\alpha}\right)_{\alpha<\omega_{1}}$ é uma sequência de conjuntos de tamanho $\omega_{1}$, então existem funções $\varphi_{1}, \varphi_{2}: \omega_{1} \longrightarrow \mathcal{P}(\kappa) \backslash\{\emptyset\}$ e uma sequência $\left\{x_{\alpha}: \alpha<\omega_{1}\right\}$, com $x_{\alpha} \in \Pi_{\beta<\alpha} X_{\beta}$ tais que:

1. Para todo $\beta \leq \alpha<\omega_{1}$ e $i, j \in\{1,2\}$, temos que ou $\varphi_{j}(\alpha) \subseteq \varphi_{i}(\beta)$ ou $\varphi_{j}(\alpha) \cap \varphi_{i}(\beta)=\emptyset$;

2. Para todo $x \in \Pi_{\alpha<\omega_{1}} X_{\alpha}$ e $\xi, \eta<\kappa$ o conjunto

$$
\begin{aligned}
& \left\{\alpha<\omega_{1}: x \mid \alpha=x_{\alpha}, \xi \in \varphi_{1}(\alpha), \eta \in \varphi_{2}(\alpha) \text { e, se } \xi \neq \eta, \varphi_{1}(\alpha) \neq \varphi_{2}(\alpha)\right\} \\
& \text { é estacionário. }
\end{aligned}
$$

Demonstração. Assumamos o princípio $\diamond$. Então, pelo Lema 3.3 sabemos que vale $\mathrm{CH}$, isto é, sabemos que $\omega_{1}=2^{\omega}$. Além disso, se $\left(X_{\alpha}\right)_{\alpha<\omega_{1}}$ é uma sequência de conjuntos de tamanho $\omega_{1}=2^{\omega}$, o item $(b)$ do Lema 3.4 nos diz que existe uma sequência $\left(t_{\alpha}, s_{\alpha}, x_{\alpha}\right)_{\alpha<\omega_{1}}$ tal que $t_{\alpha}, s_{\alpha} \in{ }^{\alpha} 2, x_{\alpha} \in \Pi_{\beta<\alpha} X_{\beta}$ e 
para todo $t, s \in{ }^{\omega_{1}} 2$ e $x \in \Pi_{\alpha<\omega_{1}} X_{\alpha}$, o conjunto

$$
\left\{\alpha<\omega_{1}: t\left|\alpha=t_{\alpha}, s\right| \alpha=s_{\alpha} \text { e } x \mid \alpha=x_{\alpha}\right\}
$$

é estacionário.

Agora, para cada $\alpha<\omega_{1}=2^{\omega}$, defina $\varphi_{1}(\alpha)=\left\{t \in{ }^{\omega_{1}} 2: t \mid \alpha=t_{\alpha}\right\}$, $\varphi_{2}(\alpha)=\left\{s \in{ }^{\omega_{1}} 2: s \mid \alpha=s_{\alpha}\right\}$ e considere a sequência $\left\{x_{\alpha}: \alpha<\omega_{1}\right\}$. Para ver que vale a condição 1 , note que se $\beta \leq \alpha$ e $t_{\alpha} \mid \beta=t_{\beta}$, então $\varphi_{1}(\alpha) \subseteq \varphi_{1}(\beta)$, e se $t_{\alpha} \mid \beta \neq t_{\beta}$, então $\varphi_{1}(\alpha) \cap \varphi_{1}(\beta)=\emptyset$. Com efeito, suponha $\beta \leq \alpha$ e $t_{\alpha} \mid \beta=t_{\beta}$. Tomando $t \in \varphi_{1}(\alpha)$ segue que $t$ é uma função de $\omega_{1}$ em 2 que satisfaz $t \mid \alpha=t_{\alpha}$. Como $\beta \leq \alpha$ temos $t|\beta=(t \mid \alpha)| \beta=t_{\alpha} \mid \beta=t_{\beta}$, provando que $t \in \varphi_{1}(\beta)$ donde segue a inclusão desejada. Suponha agora $t_{\alpha} \mid \beta \neq t_{\beta}$. Se $\varphi_{1}(\alpha) \cap \varphi_{1}(\beta) \neq \emptyset$ existiria função $t \in \omega^{\omega_{1}} 2$ satisfazendo $t \mid \alpha=t_{\alpha}$ e $t \mid \beta=t_{\beta}$ e, como $\beta \leq \alpha$, teríamos $t_{\beta}=t|\beta=(t \mid \alpha)| \beta=t_{\alpha} \mid \beta$, o que é uma contradição. O mesmo argumento pode ser repetido para outras combinações de $\varphi_{1}$ e $\varphi_{2}$ : Se $\beta \leq \alpha$ e $s_{\alpha} \mid \beta=s_{\beta}$, então $\varphi_{2}(\alpha) \subseteq \varphi_{2}(\beta)$, e se $s_{\alpha} \mid \beta \neq s_{\beta}$, então $\varphi_{2}(\alpha) \cap \varphi_{2}(\beta)=\emptyset$. Se $\beta \leq \alpha$ e $t_{\alpha} \mid \beta=s_{\beta}$, então $\varphi_{1}(\alpha) \subseteq \varphi_{2}(\beta)$, e se $t_{\alpha} \mid \beta \neq s_{\beta}$, então $\varphi_{1}(\alpha) \cap \varphi_{2}(\beta)=\emptyset$. Por fim, se $\beta \leq \alpha$ e $s_{\alpha} \mid \beta=t_{\beta}$, então $\varphi_{2}(\alpha) \subseteq \varphi_{1}(\beta)$, e se $s_{\alpha} \mid \beta \neq t_{\beta}$, então $\varphi_{2}(\alpha) \cap \varphi_{1}(\beta)=\emptyset$.

Mostraremos agora que vale a condição 2 , lembrando que estamos identificando $2^{\omega_{1}}$ com o conjunto das funções de $\omega_{1}$ em 2 . Com efeito, sejam $t, s \in{ }^{\omega_{1}} 2$ $\operatorname{com} t \neq s$ e defina $\beta$ o menor ordinal em $\omega_{1}$ satisfazendo $t(\beta) \neq s(\beta)$. Como o conjunto $\left\{\alpha<\omega_{1}: \alpha>\beta\right\}$ é fechado ilimitado e $(*)$ é estacionário, o Lema 3.2 nos diz que a intersecção desses conjuntos é ainda um conjunto estacionário.

\subsection{Resultados auxiliares}

Lema 3.7. Sejam $K$ um espaço topológico compacto $e\left(f_{n}\right)_{n \in \mathbb{N}}$ uma sequência de funções contínuas duas a duas disjuntas de $K$ em [0,1]. Temos então $\Delta\left(\left(f_{n}\right)_{n \in a}\right) \subset \Delta\left(\left(f_{n}\right)_{n \in b}\right)$ para todos subconjuntos infinitos $a \subset b \subset \mathbb{N}$.

Demonstração. Como vale que $\Delta\left(\left(f_{n}\right)_{n \in a}\right)=K \backslash D\left(\left(f_{n}\right)_{n \in a}\right)$ e também que $\Delta\left(\left(f_{n}\right)_{n \in b}\right)=K \backslash D\left(\left(f_{n}\right)_{n \in b}\right)$, basta mostrar que $D\left(\left(f_{n}\right)_{n \in b}\right) \subset D\left(\left(f_{n}\right)_{n \in a}\right)$. 
Seja, pois, $x \in D\left(\left(f_{n}\right)_{n \in b}\right)$. Então existe um aberto $U$ de $K$ contendo $x$ tal que $\left\{n \in b: U \cap \operatorname{supp}\left(f_{n}\right) \neq \emptyset\right\}$ é finito. Como $a \subset b$, temos também que $\left\{n \in a: U \cap \operatorname{supp}\left(f_{n}\right) \neq \emptyset\right\}$ é finito, donde segue que $x \in D\left(\left(f_{n}\right)_{n \in a}\right)$. Isto encerra a prova do Lema.

A Proposição a seguir é uma pequena variação do Teorema 5.1 de [Fa2] e será bastante importante na construção dos espaços $K_{\xi}$. Dizemos que uma sequência de fechados $\left(F_{n}\right)_{n \in \mathbb{N}}$ converge a um ponto $x$ se para toda vizinhança aberta $U$ de $x$ temos $F_{n} \subseteq U$ para todos, exceto finitos, $n \in \mathbb{N}$.

Proposição 3.8. Seja K um espaço métrico compacto sem pontos isolados. Dados

(a) Uma sequência de funções contínuas duas a duas disjuntas $\left(f_{n}: n \in \mathbb{N}\right)$ de $K$ em $[0,1]$;

(b) Uma sequência relativamente discreta $\left(x_{n}\right)_{n \in \mathbb{N}}$ de pontos distintos de $K$ tais que $x_{n} \notin \operatorname{supp}\left(f_{m}\right)$, para todos $n, m \in \mathbb{N}$;

(c) Um conjunto enumerável $\mathcal{P}$ de pares $\left(\left(F_{n}\right)_{n \in \mathbb{N}},\left(G_{n}\right)_{n \in \mathbb{N}}\right)$ tais que $\left(F_{n}\right)_{n \in \mathbb{N}}$ $e\left(G_{n}\right)_{n \in \mathbb{N}}$ são sequências de subconjuntos fechados disjuntos de $K$ que convergem para o mesmo ponto de $K$;

(d) $U m \varepsilon>0$;

(e) Uma sequência limitada $\left(\mu_{n}: n \in \mathbb{N}\right)$ de medidas regulares sobre $K$ tais que $\left|\int f_{n} d \mu_{n}\right|>\varepsilon$, para todo $n \in \mathbb{N}$.

existem $\delta>0, b^{\prime} \subset a \subset \mathbb{N}$ infinitos e funções contínuas $f_{n}^{\prime}: K \longrightarrow[0,1]$ tais que $\operatorname{supp}\left(f_{n}^{\prime}\right) \subset \operatorname{supp}\left(f_{n}\right)$ e para todo $b \subseteq b^{\prime}$ temos:

(f) $\left|\int f_{n}^{\prime} d \mu_{n}\right|>\delta$ e $\Sigma\left\{\int f_{m}^{\prime} d\left|\mu_{n}\right|: m \neq n, m \in a\right\}<\delta / 3$, para todo $n \in a$;

(g) $L=K\left(\left(f_{n}^{\prime}\right)_{n \in b}\right)$ é uma extensão completa;

(h) $\left(\pi_{L, K}^{-1}\left[x_{n}\right]\right)_{n \in b} e\left(\pi_{L, K}^{-1}\left[x_{n}\right]\right)_{n \in a \backslash b}$ convergem para o mesmo ponto em $L$;

(i) Para todo $\left(\left(F_{n}\right)_{n \in N},\left(G_{n}\right)_{n \in N}\right) \in \mathcal{P}$ existem $N^{\prime}, N^{\prime \prime} \subset \mathbb{N}$ infinitos e $z \in L$ tais que $\left(\pi_{L, K}^{-1}\left[F_{n}\right]\right)_{n \in N^{\prime}} e\left(\pi_{L, K}^{-1}\left[G_{n}\right]\right)_{n \in N^{\prime \prime}}$ convergem para $z$. 
Demonstração. Usando a metrizabilidade de $K$ existe $a^{\prime} \subset \mathbb{N}$ infinito tal que $\left(x_{n}\right)_{n \in a^{\prime}}$ é uma sequência convergente. Pelo lema 2.11 existem subconjuntos infinitos $b^{\prime \prime} \subset a \subset a^{\prime} \subset \mathbb{N}$ e funções contínuas $\left(f_{n}^{\prime}\right)_{n \in a}$ satisfazendo o item $(f)$ e o fato de que $L=K\left(\left(f_{n}^{\prime}\right)_{n \in b^{\prime}}\right)$ é uma extensão completa, para todo $b^{\prime} \subset b^{\prime \prime}$. Além disso, tomando $Z$ o conjunto de todos os limites de $\left(F_{n}\right)_{n \in \mathbb{N}}$ e $\left(G_{n}\right)_{n \in \mathbb{N}}$, para todo $\left(\left(F_{n}\right)_{n \in \mathbb{N}},\left(G_{n}\right)_{n \in \mathbb{N}}\right) \in \mathcal{P}$, pelo item $(g)$ do lema 2.11 podemos assumir, sem perda de generalidade, que $\Delta\left(\left(f_{n}^{\prime}\right)_{n \in b^{\prime \prime}}\right)$ é unitário ou disjunto de $Z \cup\left\{x_{n}: n \in \mathbb{N}\right\} \cup\left\{\lim _{n \in a^{\prime}} x_{n}\right\}$. Podemos assumir também que $b^{\prime \prime}$ é co-infinito em $a$.

Resta-nos provar os itens $(h)$ e $(i)$. Em outras palavras, temos que provar que algumas sequências que não podem ser separadas em $K$ continuam não se separando em $L$.

Pelo lema 3.3 de [Fa2], se $L$ é uma extensão de $K$ por $\left(f_{n}^{\prime}\right)_{n \in b^{\prime}}$, para algum $b^{\prime} \subset b^{\prime \prime}$, então $\left|\pi_{L, K}^{-1}(x)\right|=1$ para todo $x \notin \Delta\left(\left(f_{n}^{\prime}\right)_{n \in b^{\prime}}\right)$.

Agora nós vamos separar nossa construção em dois casos, lembrando que pelo Lema 3.7 o mesmo vale para todo $b^{\prime} \subset b^{\prime \prime}$ :

Caso $1 \Delta\left(\left(f_{n}^{\prime}\right)_{n \in b^{\prime \prime}}\right)$ é disjunto de $Z \cup\left\{x_{n}: n \in \mathbb{N}\right\} \cup\left\{\lim _{n \in a^{\prime}} x_{n}\right\}$.

Sejam $\left(\left(F_{n}\right)_{n \in \mathbb{N}},\left(G_{n}\right)_{n \in \mathbb{N}}\right) \in \mathcal{P}$ e $z$ o limite de ambas as sequências. Tome $z^{\prime}$ o único ponto tal que $\pi_{L, K}\left(z^{\prime}\right)=z$. Como $L$ é o gráfico de uma função contínua quando restrita a uma vizinhança aberta de $z$, temos que $\pi^{-1}\left[F_{n}\right]$ e $\pi^{-1}\left[G_{n}\right]$ convergem para $z^{\prime}$, em $L$. Isso prova o item $(i)$, e o item $(h)$ é provado analogamente.

Caso $2 \Delta\left(\left(f_{n}^{\prime}\right)_{n \in b^{\prime \prime}}\right)$ é unitário.

Seja $y$ o único ponto em tal conjunto. Então $\operatorname{supp}\left(f_{n}^{\prime}\right) \stackrel{n \in b^{\prime \prime}}{\longrightarrow} y$. De fato, se não fosse verdade, existiria uma vizinhança aberta $V$ de $y$ e um subconjunto infinito $c \subset b^{\prime \prime}$ tal que, para todo $n \in c$, existe $y_{n} \in \operatorname{supp}\left(f_{n}^{\prime}\right) \backslash V$. Tomando $y^{\prime}$ ponto de acumulação de $\left\{y_{n}: n \in c\right\}$ teríamos que $y^{\prime} \in \Delta\left(\left(f_{n}^{\prime}\right)_{n \in b^{\prime \prime}}\right)$ e $y^{\prime} \neq y$, contradizendo que $\Delta\left(\left(f_{n}^{\prime}\right)_{n \in b^{\prime \prime}}\right)$ é unitário.

Para simplificar a notação assumiremos que $\left(\left(\left\{x_{k_{n}}\right\}\right)_{n \in \mathbb{N}},\left(\left\{x_{l_{n}}\right\}\right)_{n \in \mathbb{N}}\right) \in \mathcal{P}$, onde $\left(k_{n}\right)_{n \in \mathbb{N}}$ e $\left(l_{n}\right)_{n \in \mathbb{N}}$ são enumerações de $a^{\prime}$ e $\mathbb{N} \backslash a^{\prime}$, respectivamente. Isto nos permite cuidar apenas do item $(i)$, sendo o item $(h)$ uma consequência 
imediata. Em particular, assumimos que $\lim _{n \in a^{\prime}} x_{n} \in Z$.

Se $y \notin Z$ procederemos como no Caso 1 . Para os elementos de $\mathcal{P}$ cujo limite não pertence à $Z$, também procederemos como no Caso 1 para provar o item $(i)$.

Seja $\left(\left(F_{n, m}\right)_{n \in \mathbb{N}},\left(G_{n, m}\right)_{n \in \mathbb{N}}\right)$ uma enumeração (para $m \in \mathbb{N}$ ) de todos os elementos de $\mathcal{P}$ tais que $\lim _{n \in \mathbb{N}} F_{n, m}=\lim _{n \in \mathbb{N}} G_{n, m}=y$. Esta enumeração pode ter repetições, então não precisaremos trabalhar à parte para considerar o caso em que existam apenas uma quantidade finita de elementos em $\mathcal{P}$ nestas condições.

Passando a uma subsequência, podemos assumir que $y \notin s u p p\left(f_{n}^{\prime}\right)$, que $y \notin F_{n, m}$ e $y \notin G_{n, m}$, para todos $n, m$.

Precisaremos agora da seguinte Afirmação:

Afirmação 3.8.1. Existem subconjuntos infinitos $b^{\prime}, c_{m}, d_{m} \subset \mathbb{N}$, para $m \in$ $\mathbb{N}$, tais que $F_{i, m} \cap \operatorname{supp}\left(f_{k}^{\prime}\right)=\emptyset$ e $G_{j, m} \cap \operatorname{supp}\left(f_{k}^{\prime}\right)=\emptyset$ para todos $i \in c_{m}$, $j \in d_{m}, m \in \mathbb{N}$ e $k \in b^{\prime}$.

Demonstração: Seja $U_{0}$ qualquer vizinhança aberta de $y$. Suponha que tenhamos definido $U_{n},\left(k_{j}\right)_{j<n}$ e $\left(l_{j}\right)_{j<n}$ (Os $l_{j}^{\prime} s$ são tomados em $\left.b^{\prime \prime}\right)$. Tomamos $k_{n}$ tal que $k_{n}>k_{j}$, para todo $j<n$, e $F_{k_{n}, m} \subset U_{n}$, para todo $m \leq n$. Seja $V_{n} \subset U_{n}$ uma vizinhança aberta de $y$ disjunta de $F_{k_{j}, m}$, para todos $j \leq n$ e $m \leq j$. Tome $l_{n} \in b^{\prime \prime}$ tal que $l_{n}>l_{j}$, para todo $j<n$, e $\operatorname{supp}\left(f_{l_{n}}^{\prime}\right) \subset V_{n}$. Seja $U_{n+1}$ uma vizinhança aberta de $y$ disjunta de $\operatorname{supp}\left(f_{l_{n}}^{\prime}\right)$.

Defina $\tilde{b}=\left\{l_{n}: n \in \mathbb{N}\right\}$ e $c_{m}=\left\{k_{n}: n \geq m\right\}$. Para todos $m, j \in \mathbb{N}$ e $n \geq m$. temos que $F_{k_{n}, m} \subset U_{n} \backslash V_{n}$ e $\operatorname{supp}\left(f_{l_{j}}^{\prime}\right) \subset V_{j} \backslash U_{j+1}$. Se $n \leq j$ então $F_{k_{n}, m} \cap V_{n}=\emptyset$ e $\operatorname{supp}\left(f_{l_{j}}^{\prime}\right) \subset V_{j} \subset V_{n}$. Se $n>j$ então $F_{k_{n}, m} \subset U_{n} \subset U_{j}$ e $\operatorname{supp}\left(f_{l_{j}}^{\prime}\right) \cap U_{j}=\emptyset$. Em ambos os casos temos $F_{k_{n}, m} \cap \operatorname{supp}\left(f_{l_{j}}^{\prime}\right)=\emptyset$. Procedendo analogamente para $G_{n, m}$, nós obtemos $b^{\prime} \subseteq \tilde{b}$ e $d_{m}$, o que encerra a prova da Afirmação.

Tome $b \subset b^{\prime}$, defina $L=K\left(\left(f_{n}^{\prime}\right)_{n \in b}\right)$ e denote $\pi_{L, K}$ por $\pi$.

Pela Afirmação, temos que $\lim _{n \in c_{m}} \pi^{-1}\left[F_{n, m}\right]=(y, 0)$ e também que $\lim _{n \in d_{m}} \pi^{-1}\left[G_{n, m}\right]=(y, 0)$, para todo $m \in \mathbb{N}$.

Suponha agora que $\left(F_{n}\right)_{n \in \mathbb{N}}$ e $\left(G_{n}\right)_{n \in \mathbb{N}}$ convirjam para um ponto $z$ distinto 
de $y$. Então, como no Caso $1, \pi^{-1}$ é um homeomorfismo em uma vizinhança aberta de $z$. Portanto, $\pi^{-1}\left[F_{n}\right]$ e $\pi^{-1}\left[G_{n}\right]$ convergem ambos para $\pi^{-1}(z)$, encerrando a prova da Proposição.

\subsection{Construção dos espaços $K_{\xi}$}

Assuma $\diamond$. Usando o Lema 3.6 fixamos enumerações $\left\{f_{n}(\alpha): n \in \omega\right\}$, $\left\{g_{n}(\alpha): n \in \omega\right\}, \varepsilon(\alpha),\left\{\mu_{n}(\alpha): n \in \omega\right\},\left\{x_{n}(\alpha): n \in \omega\right\}, i(\alpha), U_{\alpha}, V_{\alpha}, A_{\alpha}$, $\mathcal{B}_{\alpha}$, para $\alpha \in \omega_{1}$, e funções $\varphi_{1}$ e $\varphi_{2}$ tais que :

A.1. $\left\{f_{n}(\alpha): n \in \omega\right\}$ são funções contínuas de $[0,1]^{\omega_{1}}$ em $[0,1]$;

A.2. $\left\{g_{n}(\alpha): n \in \omega\right\}$ são funções contínuas de $[0,1]^{\omega_{1}}$ em $[0,1]$;

A.3. $\varepsilon(\alpha)>0$;

A.4. $\left(\mu_{n}(\alpha)\right)_{n \in \omega}$ é uma sequência limitada de funções de $\mathcal{B}_{\alpha}$ em $\mathbb{R}$;

A.5. $\left(x_{n}(\alpha)\right)_{n \in \omega}$ é uma sequência de pontos de $[0,1]^{\alpha}$;

A.6. $\varphi_{1}, \varphi_{2}: \omega_{1} \longrightarrow \mathcal{P}\left(2^{2^{\omega}}\right) \backslash\{\emptyset\} ;$

A.7. Para todo $\beta \leq \alpha<\omega_{1}$ e $i, j \in\{1,2\}$, temos que ou $\varphi_{j}(\alpha) \subseteq \varphi_{i}(\beta)$ ou $\varphi_{j}(\alpha) \cap \varphi_{i}(\beta)=\emptyset$;

A.8. $i(\alpha) \in\{0,1\}$;

A.9. $U_{\alpha}$ e $V_{\alpha}$ são uniões enumeráveis de abertos elementares de $[0,1]^{\omega_{1}}$ tais que $U_{\alpha} \cap V_{\alpha}=\emptyset$ e $\bar{U}_{\alpha} \cap \bar{V}_{\alpha} \neq \emptyset$;

A.10. $A_{\alpha}$ e $B_{\alpha}$ são subconjuntos enumeráveis de $[0,1]^{\alpha}$;

e, dados

B.1. uma sequência $\left\{f_{n}: n \in \omega\right\}$ de funções contínuas de $[0,1]^{\omega_{1}}$ em $[0,1]$;

B.2. uma sequência $\left\{g_{n}: n \in \omega\right\}$ de funções contínuas de $[0,1]^{\omega_{1}}$ em $[0,1]$;

B.3. $\operatorname{um} \varepsilon>0$; 
B.4. uma sequência $\left\{\mu_{n}: n \in \omega\right\}$ limitada de funções de $\mathcal{B}_{\omega_{1}}$ em $\mathbb{R}$ que representam medidas de Radon;

B.5. uma sequência $\left(x_{n}\right)_{n \in \omega}$ relativamente discreta em $[0,1]^{\omega_{1}}$;

B.6. um $\xi<\omega_{1}$;

B.7. um $\eta<\omega_{1}$;

B.8. um $i \in\{0,1\}$;

B.9. $U$ e $V$ uniões enumeráveis de abertos elementares de $[0,1]^{\omega_{1}}$ tais que $U \cap V=\emptyset$ е $\bar{U} \cap \bar{V} \neq \emptyset ;$

B.10. $\left(x_{\beta}\right)_{\beta<\omega_{1}}$ e $\left(y_{\beta}\right)_{\beta<\omega_{1}}$ seqüências em $[0,1]^{\omega_{1}}$;

o conjunto dos $\alpha<\omega_{1}$ tais que

C.1. $f_{n}(\alpha)=f_{n}$, para todo $n$;

C.2. $g_{n}(\alpha)=g_{n}$, para todo $n$;

C.3. $\varepsilon(\alpha)=\varepsilon$;

C.4. $\mu_{n}(\alpha)=\mu_{n} \mid \mathcal{B}_{\alpha}$, para todo $n$;

C.5. $x_{n}(\alpha)=x_{n} \mid \alpha$, para todo $n$;

C.6. $\xi \in \varphi_{1}(\alpha)$;

C.7. $\eta \in \varphi_{2}(\alpha)$ e $\varphi_{1}(\alpha) \neq \varphi_{2}(\alpha)$, se $\xi \neq \eta$;

C.8. $i(\alpha)=i$;

C.9. $U_{\alpha}=U$ e $V_{\alpha}=V$;

C.10. $\left\{x_{\beta} \mid \alpha: \beta \in \alpha\right\}=A_{\alpha}$ e $\left\{y_{\beta} \mid \alpha: \beta \in \alpha\right\}=B_{\alpha}$.

é estacionário.

Temos agora três casos para analisar: 
Caso $1 i(\alpha)=0$ e $\varphi_{1}(\alpha)=\varphi_{2}(\alpha)$;

Caso $2 i(\alpha)=1$ e $\varphi_{1}(\alpha)=\varphi_{2}(\alpha)$

Caso $3 \varphi_{1}(\alpha) \cap \varphi_{2}(\alpha)=\emptyset$.

No Caso 1 vamos matar operadores em $C\left(K_{\xi}(\alpha)\right)$ que não são multiplicadores fracos, para $\xi \in \varphi_{1}(\alpha)$. Já no Caso 2 vamos matar os abertosborboleta de $K_{\xi}(\alpha)$, para $\xi \in \varphi_{1}(\alpha)$. E por fim, no Caso 3, vamos matar operadores de $C\left(K_{\xi}(\alpha)\right)$ em $C\left(K_{\eta}(\alpha)\right)$ que não são fracamente compactos, para $\xi \in \varphi_{1}(\alpha)$ e $\eta \in \varphi_{2}(\alpha)$. A condição 2 do Lema 3.6 nos garante que mataremos todos os operadores que não são fracamente compactos de qualquer $C\left(K_{\xi}\right)$ em qualquer $C\left(K_{\eta}\right)$, e nos garante que mataremos todos os abertosborboleta de qualquer $K_{\xi}$ e todos os operadores que não são multiplicadores fracos de qualquer $C\left(K_{\xi}\right)$.

Seja $\kappa=2^{\omega_{1}}=2^{2^{\omega}}$. Vamos agora proceder na construção indutiva dos compactos conexos. A construção será muito similar àquela feita por Fajardo, em [Fa2] : construíremos, por indução em $\alpha<\omega_{1}=2^{\omega}$, sequências $\left(K_{\xi}(\alpha)\right)_{\xi \in \kappa, \alpha<\omega_{1}}$ de compactos conexos $K_{\xi}(\alpha) \subseteq[0,1]^{\alpha}$, sequências de conjuntos $\left(I_{\xi}(\alpha)\right)_{\xi \in \kappa, \alpha<\omega_{1}} \operatorname{com} I_{\xi}(\alpha) \subseteq \alpha \times\{0,1\}$, sequências de promessas $\left(P_{\xi}(\alpha)\right)_{\xi \in \kappa, \alpha<\omega_{1}}$ tais que $P_{\xi}(\alpha)=\left\{\left(L_{(\beta, i)}^{\alpha, \xi}, R_{(\beta, i)}^{\alpha, \xi}, z_{(\beta, i)}^{\alpha, \xi}\right):(\beta, i) \in I_{\xi}(\alpha)\right\}$, onde $L_{(\beta, i)}^{\alpha, \xi}, R_{(\beta, i)}^{\alpha, \xi} \subset \mathbb{N}$ são disjuntos e $z_{(\beta, i)}^{\alpha, \xi} \in K_{\xi}(\alpha)$, e subconjuntos fechados $F_{n}^{\beta, \xi}(\alpha) \subset K_{\xi}(\alpha)$, para $\beta$ satisfazendo $(\beta, 0) \in I_{\xi}(\alpha)$.

Uma vez definido $K_{\xi}(\alpha)$, para todo $\beta$ tal que $(\beta, 0) \in I_{\xi}(\alpha)$ definiremos

$$
F_{n}^{\beta, \xi}(\alpha)=\pi_{K_{\xi}(\alpha), K_{\xi}(\beta)}^{-1}\left[\left\{x_{n}(\beta)\right\}\right]
$$

Suponha que tenhamos $\left(K_{\xi}(\gamma)\right)_{\xi<\kappa, \gamma<\alpha}$ e $\left(P_{\xi}(\gamma)\right)_{\xi<\kappa, \gamma<\alpha}$ satisfazendo as seguintes condições indutivas, para todo $\gamma<\alpha$ :

D.1. $\operatorname{Se}(\beta, i) \in I_{\xi}(\alpha)$ e $\gamma>\beta$, temos $\lim _{n \in L_{(\beta, i)}^{\gamma, \xi}} F_{n}^{\beta, \xi}(\gamma)=\lim _{n \in R_{(\beta, i)}^{\gamma, \xi}} F_{n}^{\beta, \xi}(\gamma)=$ $z_{(\beta, i)}^{\gamma, \xi}$

D.2. Se $(\beta, i) \in I_{\xi}(\alpha)$ e $\gamma>\gamma^{\prime}>\beta$, temos $\pi_{\gamma^{\prime}}\left[K_{\xi}(\gamma)\right]=K_{\xi}\left(\gamma^{\prime}\right)$ e $z_{(\beta, i)}^{\gamma, \xi} \mid \gamma^{\prime}=$ $z_{(\beta, i)}^{\gamma^{\prime}, \xi}$; 
D.3. Para todo $(\beta, i) \in I_{\xi}(\alpha)$ e todos $\gamma>\gamma^{\prime}>\beta$, os conjuntos $L_{(\beta, i)}^{\gamma, \xi} \backslash L_{(\beta, i)}^{\gamma^{\prime}, \xi}$ e $R_{(\beta, i)}^{\gamma, \xi} \backslash R_{(\beta, i)}^{\gamma^{\prime}, \xi}$ são finitos.

Se $\alpha$ é um ordinal limite, definimos

E.1. $K_{\xi}(\alpha)$ é o limite inverso $\left(K_{\xi}(\gamma)\right)_{\gamma<\alpha}$;

E.2. $I_{\xi}(\alpha)=\bigcup_{\beta<\alpha} I_{\xi}(\beta)$;

E.3. $z_{(\beta, i)}^{\alpha, \xi}=\bigcup_{\beta<\gamma<\alpha} z_{(\beta, i)}^{\gamma, \xi}$, para todo $(\beta, i) \in I_{\xi}(\alpha)$;

E.4. Para todo $(\beta, i) \in I_{\xi}(\alpha), L_{(\beta, i)}^{\alpha, \xi}$ é uma pseudointersecção infinita de $\left(L_{(\beta, i)}^{\gamma, \xi}\right)_{\beta<\gamma<\alpha}$, isto é, o conjunto $L_{(\beta, i)}^{\alpha, \xi} \backslash L_{(\beta, i)}^{\gamma, \xi}$ é finito, para todo $\gamma<\alpha$ (a existência de tal pseudointersecção é provada em [Do, Teorema 3.1]);

E.5. Para todo $(\beta, i) \in I_{\xi}(\alpha), R_{(\beta, i)}^{\alpha, \xi}$ é uma pseudointersecção infinita de $\left(R_{(\beta, i)}^{\gamma, \xi}\right)_{\beta<\gamma<\alpha}$.

Vamos agora trabalhar no caso ordinal sucessor. Suponha que tenhamos definido $\left(K_{\xi}(\gamma)\right)_{\gamma \leq \alpha}$ e $\left(P_{\xi}(\gamma)\right)_{\gamma \leq \alpha}$. Vamos definir $K_{\xi}(\alpha+1)$ e $P_{\xi}(\alpha+1)$.

Diremos que $\alpha<\omega_{1}$ é não trivial se vale um dos seguintes três casos:

1) $i(\alpha)=0, \varphi_{1}(\alpha)=\varphi_{2}(\alpha)$ e além disso, para $\xi \in \varphi_{1}(\alpha)$ fixado temos:

F.1. $\left(x_{n}(\alpha)\right)_{n \in \mathbb{N}}$ é uma sequência relativamente discreta de pontos distintos de $K_{\xi}(\alpha)$;

F.2. Existem funções contínuas $f_{n}^{\prime}:[0,1]^{\alpha} \longrightarrow[0,1]$ tais que $f_{n}(\alpha)=f_{n}^{\prime} \circ \pi_{\alpha}$;

F.3. $\left(\left.f_{n}^{\prime}\right|_{K_{\xi}(\alpha)}: n \in \mathbb{N}\right)$ como antes é disjunta;

F.4. $x_{n}(\alpha) \notin \operatorname{supp}\left(f_{m}^{\prime}\right)$, para todo $n, m \in \mathbb{N}$ e $f_{m}^{\prime}$ como no item F.2;

F.5. $\left|\int_{K_{\xi}(\alpha)} f_{n}^{\prime} d \mu_{n}(\alpha)\right|>\varepsilon(\alpha)$, para todo $n \in \mathbb{N}$.

2) $i(\alpha)=1, \varphi_{1}(\alpha)=\varphi_{2}(\alpha)$ e além disso, para $\xi \in \varphi_{1}(\alpha)$ fixado temos:

G.1. $A_{\alpha}, B_{\alpha} \subset K_{\xi}(\alpha)$; 
G.2. $U_{\alpha}=\pi_{\alpha}^{-1}\left[\pi_{\alpha}\left[U_{\alpha}\right]\right]$ e $V_{\alpha}=\pi_{\alpha}^{-1}\left[\pi_{\alpha}\left[V_{\alpha}\right]\right]$;

G.3. $\overline{\pi_{\alpha}\left[U_{\alpha}\right] \cap A_{\alpha}} \cap \overline{\pi_{\alpha}\left[V_{\alpha}\right] \cap B_{\alpha}} \neq \emptyset$;

G.4. $x_{n}(\alpha) \stackrel{n \in \omega}{\rightarrow} z$, para algum $z \in \overline{\pi_{\alpha}\left[U_{\alpha}\right] \cap A_{\alpha}} \cap \overline{\pi_{\alpha}\left[V_{\alpha}\right] \cap B_{\alpha}} \mathrm{e}$

$$
\begin{gathered}
\left\{x_{n}(\alpha): n \in 2 \omega\right\} \subseteq A_{\alpha} ; \\
\left\{x_{n}(\alpha): n \in \omega \backslash 2 \omega\right\} \subseteq B_{\alpha} .
\end{gathered}
$$

3) $\varphi_{1}(\alpha) \cap \varphi_{2}(\alpha)=\emptyset$ e além disso, para $\xi \in \varphi_{1}(\alpha)$ e $\eta \in \varphi_{2}(\alpha)$ fixados temos:

H.1. $\left(x_{n}(\alpha)\right)_{n \in \mathbb{N}}$ é uma sequência relativamente discreta de pontos distintos de $K_{\eta}(\alpha)$;

H.2. Existem funções contínuas $f_{n}^{\prime}:[0,1]^{\alpha} \longrightarrow[0,1]$ tais que $f_{n}(\alpha)=f_{n}^{\prime} \circ \pi_{\alpha}$;

H.3. $\left(\left.f_{n}^{\prime}\right|_{K_{\xi}(\alpha)}: n \in \mathbb{N}\right)$ como antes é disjunta;

H.4. $\left|\int_{K_{\xi}(\alpha)} f_{n}^{\prime} d \mu_{n}(\alpha)\right|>\varepsilon(\alpha)$, para todo $n \in \mathbb{N}$;

H.5. Existem funções contínuas $g_{n}^{\prime}:[0,1]^{\alpha} \longrightarrow[0,1]$ tais que $g_{n}(\alpha)=g_{n}^{\prime} \circ \pi_{\alpha}$;

H.6. $g_{n}^{\prime}\left(x_{n}(\alpha)\right)>\varepsilon(\alpha)$.

Se $\alpha<\omega_{1}$ for trivial definiremos, para $\xi<\kappa, K_{\xi}(\alpha+1)=K_{\xi}(\alpha) \times\{0\}$, $I_{\xi}(\alpha+1)=I_{\xi}(\alpha), L_{(\beta, i)}^{\alpha+1, \xi}=L_{(\beta, i)}^{\alpha, \xi}, R_{(\beta, i)}^{\alpha+1, \xi}=R_{(\beta, i)}^{\alpha, \xi}$ e $z_{(\beta, i)}^{\alpha+1, \xi}=z_{(\beta, i)}^{\alpha, \xi \frown} 0$, para todo $(\beta, i) \in I_{\xi}(\alpha)$.

Agora, se $\alpha<\omega_{1}$ for não trivial teremos três casos para analisar :

a) $i(\alpha)=0$ e $\varphi_{1}(\alpha)=\varphi_{2}(\alpha)$;

Nesse caso, a construção indutiva se dará da seguinte maneira : Sejam $f_{n}^{\prime}$ funções como em F.2 e defina $h_{n}=\left.f_{n}^{\prime}\right|_{K_{\xi}(\alpha)}$, para $\xi \in \varphi_{1}(\alpha)$ fixado. Pela Proposição 3.8 existem subconjuntos infinitos $b \subset a \subset \mathbb{N}$, um $\delta>0$ e funções contínuas $h_{n}^{\prime}: K_{\xi}(\alpha) \longrightarrow[0,1]$, para $n \in a$, tais que:

I.1. $\operatorname{supp}\left(h_{n}^{\prime}\right) \subset \operatorname{supp}\left(h_{n}\right)$, para todo $n \in a$;

I.2. $K_{\xi}(\alpha+1)=K_{\xi}(\alpha)\left(\left(h_{n}^{\prime}\right)_{n \in b}\right)$ é uma extensão completa; 
I.3. $\left|\int h_{n}^{\prime} d \mu_{n}(\alpha)\right|>\delta$ e $\Sigma\left\{\int h_{m}^{\prime} d\left|\mu_{n}(\alpha)\right|: m \neq n, m \in a\right\}<\frac{\delta}{3}$, para todo $n \in a$;

I.4. Para todo $(\beta, i) \in I_{\xi}(\alpha)$ existem subconjuntos de tamanho infinito $L_{(\beta, i)}^{\alpha+1, \xi} \subset L_{(\beta, i)}^{\alpha, \xi}$ e $R_{(\beta, i)}^{\alpha+1, \xi} \subset R_{(\beta, i)}^{\alpha, \xi}$ e um ponto $z_{(\beta, i)}^{\alpha+1, \xi}$ tais que $\lim _{n \in L_{(\beta, i)}^{\alpha+1, \xi}} \pi^{-1}\left[F_{n}^{\beta, \xi}(\alpha)\right]=\lim _{n \in R_{(\beta, i)}^{\alpha+1, \xi}} \pi^{-1}\left[F_{n}^{\beta, \xi}(\alpha)\right]=z_{(\beta, i)}^{\alpha+1, \xi}$, onde $\pi$ é a projeção standard de $K_{\xi}(\alpha+1)$ em $K_{\xi}(\alpha)$;

I.5. $\left(\pi^{-1}\left[x_{n}(\alpha)\right]\right)_{n \in b}$ e $\left(\pi^{-1}\left[x_{n}(\alpha)\right]\right)_{n \in a \backslash b}$ convergem para um mesmo ponto $z_{(\alpha, 0)}^{\alpha+1, \xi} \in K_{\xi}(\alpha+1)$.

Definimos $I_{\xi}(\alpha+1)=I_{\xi}(\alpha) \cup\{(\alpha, 0)\}, L_{(\alpha, 0)}^{\alpha+1, \xi}=b$ e $R_{(\alpha, 0)}^{\alpha+1, \xi}=a \backslash b$. Por fim, tomamos $F_{n}^{\alpha, \xi}(\alpha)=\left\{x_{n}(\alpha)\right\}$ e $F_{n}^{\beta, \xi}(\alpha+1)=\pi^{-1}\left[F_{n}^{\beta, \xi}(\alpha)\right]$, para todo $n \in \mathbb{N}$ e todo $\beta$ satisfazendo $(\beta, 0) \in I_{\xi}(\alpha)$.

Isso completa a construção indutiva de $K_{\xi}(\alpha+1)$ no caso a), para $\xi \in \varphi_{1}(\alpha)$. No caso em que $\zeta<\kappa$ é tal que $\zeta \notin \varphi_{1}(\alpha)$, definimos $K_{\zeta}(\alpha+1), I_{\zeta}(\alpha+1)$ e $P_{\zeta}(\alpha+1)$ como no caso trivial.

b) $i(\alpha)=1$ e $\varphi_{1}(\alpha)=\varphi_{2}(\alpha)$;

Nesse caso, construíremos da seguinte maneira : Tome $z=\lim _{n \rightarrow \infty} x_{n}(\alpha)$ em $K_{\xi}(\alpha)$, onde $\xi \in \varphi_{1}(\alpha)$ está fixado. Pelo lema de Urysohn existe uma sequência de funções contínuas duas a duas disjuntas $\left(h_{n}\right)_{n \in 2 \mathbb{N}}$ de $K_{\xi}(\alpha)$ em $[0,1]$ tais que $\Delta\left(\left(h_{n}\right)_{n \in 2 \mathbb{N}}\right)=\{z\}$ e, para todo $n \in 2 \mathbb{N}$ e $m \in \mathbb{N}$,

$$
h_{n}\left(x_{m}(\alpha)\right)= \begin{cases}1, & \text { se } m=n \text { ou } m=n+1 \\ 0, & \text { caso contrário. }\end{cases}
$$

Seja $b \subseteq 2 \mathbb{N}$ tal que $K_{\xi}(\alpha)\left(\left(h_{n}\right)_{n \in b}\right)$ é uma extensão forte. Definimos então $I_{\xi}(\alpha+1)=I_{\xi}(\alpha) \cup\{(\alpha, 0),(\alpha, 1)\}, K_{\xi}(\alpha+1)=K_{\xi}(\alpha)\left(\left(h_{n}\right)_{n \in b}\right)$, $L_{(\alpha, 1)}^{\alpha+1, \xi}=b, L_{(\alpha, 0)}^{\alpha+1, \xi}=2 \mathbb{N} \backslash b, R_{(\alpha, 1)}^{\alpha+1, \xi}=\left\{n+1: n \in L_{(\alpha, 1)}^{\alpha+1, \xi}\right\}, R_{(\alpha, 0)}^{\alpha+1, \xi}=$ $\left\{n+1: n \in L_{(\alpha, 0)}^{\alpha+1, \xi}\right\}, z_{(\alpha, 0)}^{\alpha+1, \xi}=z \frown 0$ e $z_{(\alpha, 1)}^{\alpha+1, \xi}=z \frown 1$.

Observe que $F_{n}^{\alpha, \xi}(\alpha+1)=\left\{x_{n}(\alpha)^{\frown} 1\right\}$, se $n \in L_{(\alpha, 1)}^{\alpha+1, \xi} \cup R_{(\alpha, 1)}^{\alpha+1, \xi}$, e que $F_{n}^{\alpha, \xi}(\alpha+1)=\left\{x_{n}(\alpha) \frown 0\right\}$, caso contrário. Concluímos portanto que $F_{n}^{\alpha, \xi}(\alpha+1) \rightarrow z_{(\alpha, 0)}^{\alpha+1, \xi}$, para $n \in L_{(\alpha, 0)}^{\alpha+1, \xi} \cup R_{(\alpha, 0)}^{\alpha+1, \xi}$, e $F_{n}^{\alpha, \xi}(\alpha+1) \rightarrow z_{(\alpha, 1)}^{\alpha+1, \xi}$, para $n \in L_{(\alpha, 1)}^{\alpha+1, \xi} \cup R_{(\alpha, 1)}^{\alpha+1, \xi}$. 
O restante da construção de $P_{\xi}(\alpha+1)$ - a saber, $L_{(\beta, i)}^{\alpha+1, \xi}, R_{(\beta, i)}^{\alpha+1, \xi}$ e $z_{(\beta, i)}^{\alpha+1, \xi}$, para $(\beta, i) \in I_{\xi}(\alpha)$ - é feito como no caso a), repetindo o argumento usado no Caso 2 da prova do item $(i)$ da Proposição 3.8. No caso em que $\zeta<\kappa$ é tal que $\zeta \notin \varphi_{1}(\alpha)$, definimos $K_{\zeta}(\alpha+1), I_{\zeta}(\alpha+1)$ e $P_{\zeta}(\alpha+1)$ como no caso trivial.

c) $\varphi_{1}(\alpha) \cap \varphi_{2}(\alpha)=\emptyset$.

Neste caso procederemos a construção da seguinte maneira : fixemos $\xi \in \varphi_{1}(\alpha)$ e $\eta \in \varphi_{2}(\alpha)$. Sejam então $f_{n}^{\prime}$ funções como em H.2 e defina $e_{n}=\left.f_{n}^{\prime}\right|_{K_{\xi}(\alpha)}$. Logo, existem $b^{\prime} \subset a^{\prime} \subset \mathbb{N}$ subconjuntos infinitos, $\delta>0$ e funções $e_{n}^{\prime}: K_{\xi}(\alpha) \longrightarrow[0,1]$ tais que, para todo $b \subset b^{\prime}$ valem as teses $(f),(g),(h)$ e $(i)$ da Proposição 3.8. Agora, fixe $b^{\prime \prime} \subset b^{\prime}$ infinito e co-infinito tal que

$$
\overline{\left\{x_{n}(\alpha): n \in b^{\prime \prime}\right\}} \cap \overline{\left\{x_{n}(\alpha): n \in b^{\prime} \backslash b^{\prime \prime}\right\}} \neq \emptyset .
$$

A existência de tal $b^{\prime \prime}$ segue do fato de que $K_{\eta}(\alpha)$ tem peso menor que $2^{\omega}$ e do fato de que em um espaço compacto a separação de fechados pode ser feita por uma quantidade finita de abertos básicos.

Por $(*)$, sabemos que existe $z \in \overline{\left\{x_{n}(\alpha): n \in b^{\prime \prime}\right\}} \cap \overline{\left\{x_{n}(\alpha): n \in b^{\prime} \backslash b^{\prime \prime}\right\}}$. Como $K_{\eta}(\alpha)$ é métrico, existe uma sequência em $\left\{x_{n}(\alpha): n \in b^{\prime \prime}\right\}$ e uma sequência em $\left\{x_{n}(\alpha): n \in b^{\prime} \backslash b^{\prime \prime}\right\}$, ambas convergindo para $z$. Sejam pois $b \subseteq b^{\prime \prime}$ e $a^{\prime \prime} \subseteq b^{\prime} \backslash b^{\prime \prime}$ subconjuntos infinitos correspondentes às coordenadas dessas sequências que convergem a $z$. Definimos então $I_{\eta}(\alpha+1)=I_{\eta}(\alpha) \cup\{(\alpha, 0)\}, \quad L_{(\alpha, 0)}^{\alpha+1, \eta}=b, R_{(\alpha, 0)}^{\alpha+1, \eta}=a^{\prime \prime}$ e $z_{(\alpha, 0)}^{\alpha+1, \eta}=z$. Além disso, definimos também $K_{\eta}(\alpha+1)=K_{\eta}(\alpha) \times\{0\}, L_{(\beta, i)}^{\alpha+1, \eta}=L_{(\beta, i)}^{\alpha, \eta}$, $R_{(\beta, i)}^{\alpha+1, \eta}=R_{(\beta, i)}^{\alpha, \eta}$ e $z_{(\beta, i)}^{\alpha+1, \eta}=z_{(\beta, i)}^{\alpha, \eta \frown} 0$, para todo $(\beta, i) \in I_{\xi}(\alpha)$. Por fim, tomamos $F_{n}^{\alpha, \eta}(\alpha)=\left\{x_{n}(\alpha)\right\}$ e $F_{n}^{\beta, \eta}(\alpha+1)=\pi^{-1}\left[F_{n}^{\beta, \eta}(\alpha)\right]$, para todo $n \in \mathbb{N}$ e todo $\beta$ satisfazendo $(\beta, 0) \in I_{\eta}(\alpha)$.

Seja $a=a^{\prime \prime} \cup b$ e defina $I_{\xi}(\alpha+1)=I_{\xi}(\alpha)$. Então, para $a$ e $b$ como acima temos: 
J.1. $\operatorname{supp}\left(e_{n}^{\prime}\right) \subset \operatorname{supp}\left(e_{n}\right)$, para todo $n \in a$;

J.2. $K_{\xi}(\alpha+1)=K_{\xi}(\alpha)\left(\left(e_{n}^{\prime}\right)_{n \in b}\right)$ é uma extensão completa;

J.3. $\left|\int e_{n}^{\prime} d \mu_{n}(\alpha)\right|>\delta$ e $\Sigma\left\{\int e_{m}^{\prime} d\left|\mu_{n}(\alpha)\right|: m \neq n, m \in a\right\}<\frac{\delta}{3}$, para todo $n \in a$;

J.4. Para todo $(\beta, i) \in I_{\xi}(\alpha)$ existem subconjuntos de tamanho infinito $L_{(\beta, i)}^{\alpha+1, \xi} \subset L_{(\beta, i)}^{\alpha, \xi}$ e $R_{(\beta, i)}^{\alpha+1, \xi} \subset R_{(\beta, i)}^{\alpha, \xi}$ e um ponto $z_{(\beta, i)}^{\alpha+1, \xi}$ tais que $\lim _{n \in L_{(\beta, i)}^{\alpha+1, \xi}} \pi^{-1}\left[F_{n}^{\beta, \xi}(\alpha)\right]=\lim _{n \in R_{(\beta, i)}^{\alpha+1, \xi}} \pi^{-1}\left[F_{n}^{\beta, \xi}(\alpha)\right]=z_{(\beta, i)}^{\alpha+1, \xi}$, onde $\pi$ é a projeção standard de $K_{\xi}(\alpha+1)$ em $K_{\xi}(\alpha)$;

J.5. $\left(\pi^{-1}\left[x_{n}(\alpha)\right]\right)_{n \in b}$ e $\left(\pi^{-1}\left[x_{n}(\alpha)\right]\right)_{n \in a \backslash b}$ convergem para um mesmo ponto $z_{(\alpha, 0)}^{\alpha+1, \eta} \in K_{\eta}(\alpha+1)$.

No caso em que $\zeta<\kappa$ é tal que $\zeta \notin \varphi_{1}(\alpha)$ e $\zeta \notin \varphi_{2}(\alpha)$, definimos $K_{\zeta}(\alpha+1), I_{\zeta}(\alpha+1)$ e $P_{\zeta}(\alpha+1)$ como no caso trivial.

O resultado a seguir nos mostra que a construção indutiva anteriormente realizada está bem definida.

Proposição 3.9. Para todo $\alpha<\omega_{1}$ temos $K_{\xi}(\alpha)=K_{\xi^{\prime}}(\alpha)$, sempre que $i \in\{1,2\}$ e $\xi, \xi^{\prime} \in \varphi_{i}(\alpha)$.

Demonstração. Suponha que não seja verdade que os $K_{\xi}(\alpha)$ 's são todos iguais para $\xi$ 's em $\varphi_{i}(\alpha)$. Seja então $\alpha$ o menor ordinal para o qual existem $i \in\{1,2\}$ e $\xi, \xi^{\prime} \in \varphi_{i}(\alpha)$ tais que $K_{\xi}(\alpha) \neq K_{\xi^{\prime}}(\alpha)$. Fixe tais $i, \xi$, $\xi^{\prime}$ e defina $\gamma$ o menor ordinal tal que $K_{\xi}(\gamma) \neq K_{\xi^{\prime}}(\gamma)$. Se $\gamma$ é limite temos uma contradição pois $K_{\xi}(\gamma)$ e $K_{\xi^{\prime}}(\gamma)$ são limites inversos de compactos que, pela hipótese indutiva, são os mesmos. Suponhamos agora $\gamma=\beta+1$ para algum ordinal $\beta$. Pela hipótese indutiva temos $K_{\xi}(\beta)=K_{\xi^{\prime}}(\beta)$. Suponha que $\xi \in \varphi_{j}(\beta)$, para algum $j \in\{1,2\}$. Teríamos então que $\xi \in \varphi_{j}(\beta) \cap \varphi_{i}(\alpha)$. Logo, pela condição 1 do Lema 3.6, seguiria que $\varphi_{i}(\alpha) \subseteq \varphi_{j}(\beta)$ e portanto $\xi, \xi^{\prime} \in \varphi_{j}(\beta)$. Daí, pela construção, teríamos que ter $K_{\xi}(\beta+1)=K_{\xi^{\prime}}(\beta+1)$, o que é uma contradição. Se $\xi^{\prime} \in \varphi_{j}(\beta)$, para algum $j \in\{1,2\}$, o mesmo argumento de antes nos diria que $K_{\xi}(\beta+1)=K_{\xi^{\prime}}(\beta+1)$, o que é uma contradição. Por fim, se nem $\xi$ e nem $\xi^{\prime}$ estão em $\varphi_{j}(\beta)$ para algum $j \in\{1,2\}$, teríamos que 
ter $K_{\xi}(\beta+1)=K_{\xi}(\beta) \times\{0\}=K_{\xi^{\prime}}(\beta) \times\{0\}=K_{\xi^{\prime}}(\beta+1)$, o que também é uma contradição.

Para cada $\xi \in 2^{\omega_{1}}$ definimos $K_{\xi}$ o limite inverso de $\left(K_{\xi}(\alpha)\right)_{\alpha<\omega_{1}}$.

A próxima Proposição nos traz características topológicas importantes com respeito a cada $K_{\xi}$ construído indutivamente. A demonstração é uma adaptação da demonstração do Teorema 5.2 presente em [Fa2].

Proposição 3.10. $(\diamond)$ Sejam $\xi, \eta<2^{\omega_{1}}$. Então $K_{\xi}$ é um espaço topológico compacto e conexo tal que:

(i) Dados

(a) Uma sequência $\left(f_{n}: n \in \mathbb{N}\right)$ de funções contínuas e duas a duas disjuntas de $K_{\xi}$ em $[0,1]$;

(b) Uma sequência relativamente discreta $\left(x_{n}: n \in \mathbb{N}\right)$ de pontos distintos de $K_{\eta}$ tal que, se $\xi=\eta$, então $f_{m}\left(x_{n}\right)=0$ para todos $n, m \in \mathbb{N}$;

(c) $\operatorname{Um} \varepsilon>0$;

(d) Uma sequência limitada $\left(\mu_{n}: n \in \mathbb{N}\right)$ de medidas regulares sobre $K_{\xi}$ tal que $\left|\int f_{n} d \mu_{n}\right|>\varepsilon$, para todo $n \in \mathbb{N}$.

existem $\delta>0$, subconjuntos infinitos $b \subset a \subset \mathbb{N}$ e funções contínuas $f_{n}^{\prime}, \operatorname{com} \operatorname{supp}\left(f_{n}^{\prime}\right) \subset \operatorname{supp}\left(f_{n}\right)$, tais que

(e) $\left|\int f_{n}^{\prime} d \mu_{n}\right|>\delta$ e $\Sigma\left\{\int f_{m}^{\prime} d\left|\mu_{n}\right|: m \neq n, m \in a\right\}<\delta / 3$, para todo $n \in a$;

(f) $\left(f_{n}^{\prime}\right)_{n \in b}$ tem supremo em $C\left(K_{\xi}\right)$;

(g) $\overline{\left\{x_{n}: n \in b\right\}} \cap \overline{\left\{x_{n}: n \in a \backslash b\right\}} \neq \emptyset$.

(ii) Se $L$ é um subespaço fechado de $K_{\xi}$ e $V_{1}$ e $V_{2}$ são dois abertos de $L$ disjuntos tais que $\bar{V}_{1} \cap \bar{V}_{2} \neq \emptyset$, então $\bar{V}_{1} \cap \bar{V}_{2}$ tem pelo menos dois elementos. 
Demonstração. A compacidade de $K_{\xi}$ segue do fato de que $K_{\xi}$ é o limite inverso de espaços compactos. Além disso, pelo lema 3.7 de [Fa2] temos também que $K_{\xi}$ é conexo. Vamos agora dividir a prova do item $(i)$ em dois casos:

\section{Caso $1 \quad \xi=\eta$;}

Usando o lema de Urysohn e a regularidade das medidas $\mu_{n}$ para reduzir os suportes das $f_{n}$ preservando a condição $(d)$ da hipótese da Proposição, podemos assumir sem perda de generalidade que $x_{n} \notin \operatorname{supp}\left(f_{m}\right)$, para todos $n, m \in \mathbb{N}$.

Pelo teorema de Tietze estendemos continuamente as funções $f_{n}$ para $\tilde{f}_{n}:[0,1]^{\omega_{1}} \longrightarrow[0,1]$. Pelo teorema de Mibu (veja [Mi]) existem $\alpha<\omega_{1} \mathrm{e}$ funções contínuas $g_{n}:[0,1]^{\alpha} \longrightarrow[0,1]$ tais que $\tilde{f}_{n}=g_{n} \circ \pi$. Observe que $f_{n}=\left.g_{n}\right|_{K_{\xi}(\alpha)} \circ \pi_{\alpha}$. Como a existência de tais funções continua acontecendo para todo $\alpha^{\prime}>\alpha$, pois $\tilde{f}_{n} \circ \pi_{\alpha^{\prime}}=g_{n} \circ \pi_{\alpha} \circ \pi_{\alpha^{\prime}}$, podemos escolher ordinal não trivial $\alpha<\omega_{1}, \operatorname{com} i(\alpha)=0$ e $\varphi_{1}(\alpha)=\varphi_{2}(\alpha)$ e $\xi \in \varphi_{1}(\alpha)$, tal que:

K.1. $f_{n}(\alpha)=\tilde{f}_{n}$, para todo $n \in \mathbb{N}$;

K.2. $x_{n}(\alpha)=x_{n} \mid \alpha$, para todo $n \in \mathbb{N}$;

K.3. $\varepsilon(\alpha)=\varepsilon$;

K.4. $\mu_{n}(\alpha)=\mu_{n} \mid \mathcal{B}_{\alpha}$, para todo $n \in \mathbb{N}$.

Tome $b=L_{(\alpha, 0)}^{\alpha+1, \xi}$ e $a=L_{(\alpha, 0)}^{\alpha+1, \xi} \cup R_{(\alpha, 0)}^{\alpha+1, \xi}$. Defina então $f_{n}^{\prime}=h_{n}^{\prime} \circ \pi$, onde $h_{n}^{\prime}$ são como em $\mathbf{I} . \mathbf{1}$ até $\mathbf{I} .5$, lembrando que $K_{\xi}(\alpha+1)=K_{\xi}(\alpha)\left(\left(h_{n}^{\prime}\right)_{n \in b}\right)$. Fixe $\delta>0$ como em I.3.

Pelo lema 3.4 de [Fa2], $\left(h_{n}^{\prime} \circ \pi\right)_{n \in b}$ tem supremo em $C\left(K_{\xi}(\alpha+1)\right)$. Pelo lema 3.7 de [Fa2], $\left(f_{n}^{\prime}\right)_{n \in b}$ tem supremo em $C\left(K_{\xi}\right)$ e isso prova (f).

Observe que $\int f_{n}^{\prime} d \mu_{m}=\int h_{n}^{\prime} d \mu_{m}(\alpha)$, para todo $n, m$, já que $f_{n}^{\prime}$ é determinada pelas coordenadas abaixo de $\alpha$. Logo também concluímos o item (e).

Resta-nos provar o item $(g)$. Suponha que existam $U_{1}$ e $U_{2}$ subconjuntos abertos de $K_{\xi}$ tais que $x_{n}(\alpha) \in U_{1}$, para todo $n \in b$, e $x_{n}(\alpha) \in U_{2}$, para 
todo $n \in a \backslash b$. Pela compacidade de $K_{\xi}$, podemos assumir que $U_{1}$ e $U_{2}$ são uniões finitas de abertos elementares. Portanto existe $\beta<\omega_{1}$, que pode ser escolhido maior do que $\alpha$, tal que a separação ocorre abaixo de $\beta$, i.e., $\overline{\left\{x_{n} \mid \beta: n \in b\right\}} \cap \overline{\left\{x_{n} \mid \beta: n \in a \backslash b\right\}}=\emptyset$, em $K_{\xi}(\beta)$. Como $x_{n} \mid \alpha=x_{n}(\alpha)$, te$\operatorname{mos} x_{n} \mid \beta \in F_{n}^{\alpha, \xi}(\beta)$. Pela construção temos que $L_{(\alpha, 0)}^{\beta, \xi} \backslash L_{(\alpha, 0)}^{\alpha+1, \xi}$ é finito. Como $L_{(\alpha, 0)}^{\alpha+1, \xi}=b$ e $\lim _{n \in L_{(\alpha, 0)}^{\beta, \xi}} F_{n}^{\alpha, \xi}(\beta)=z_{(\alpha, 0)}^{\beta, \xi}$, temos que $z_{(\alpha, 0)}^{\beta, \xi} \in \overline{\left\{x_{n} \mid \beta: n \in b\right\}}$. Analogamente concluímos que $z_{(\alpha, 0)}^{\beta, \xi} \in \overline{\left\{x_{n} \mid \beta: n \in a \backslash b\right\}}$, contradizendo o fato de que $\overline{\left\{x_{n} \mid \beta: n \in b\right\}} \cap \overline{\left\{x_{n} \mid \beta: n \in a \backslash b\right\}}=\emptyset$.

\section{Caso $2 \quad \xi \neq \eta$.}

Pelo teorema de Tietze estendemos continuamente as funções $f_{n}$ para $\tilde{f}_{n}:[0,1]^{\omega_{1}} \longrightarrow[0,1]$. Pelo teorema de Mibu (veja [Mi]) existem $\alpha<\omega_{1}$ e funções contínuas $g_{n}:[0,1]^{\alpha} \longrightarrow[0,1]$ tais que $\tilde{f}_{n}=g_{n} \circ \pi$. Observe que $f_{n}=\left.g_{n}\right|_{K_{\xi}(\alpha)} \circ \pi_{\alpha}$. Como a existência de tais funções continua acontecendo para todo $\alpha^{\prime}>\alpha$, pois $\tilde{f}_{n} \circ \pi_{\alpha^{\prime}}=g_{n} \circ \pi_{\alpha} \circ \pi_{\alpha^{\prime}}$, podemos escolher ordinal não trivial $\alpha<\omega_{1}$, com $\varphi_{1}(\alpha) \cap \varphi_{2}(\alpha)=\emptyset$ e $\xi \in \varphi_{1}(\alpha)$ e $\eta \in \varphi_{1}(\alpha)$, tal que:

K.1. $f_{n}(\alpha)=\tilde{f}_{n}$, para todo $n \in \mathbb{N}$;

K.2. $x_{n}(\alpha)=x_{n} \mid \alpha$, para todo $n \in \mathbb{N}$;

K.3. $\varepsilon(\alpha)=\varepsilon$;

K.4. $\mu_{n}(\alpha)=\mu_{n} \mid \mathcal{B}_{\alpha}$, para todo $n \in \mathbb{N}$.

Tome $b=L_{(\alpha, 0)}^{\alpha+1, \eta}$ e $a=L_{(\alpha, 0)}^{\alpha+1, \eta} \cup R_{(\alpha, 0)}^{\alpha+1, \eta}$. Defina então $f_{n}^{\prime}=e_{n}^{\prime} \circ \pi$, onde $e_{n}^{\prime}$ são como em $\mathbf{J} .1$ até $\mathbf{J . 5}$, lembrando que $K_{\xi}(\alpha+1)=K_{\xi}(\alpha)\left(\left(e_{n}^{\prime}\right)_{n \in b}\right)$. Fixe $\delta>0$ como em J.3.

Pelo lema 3.4 de [Fa2], $\left(e_{n}^{\prime} \circ \pi\right)_{n \in b}$ tem supremo em $C\left(K_{\xi}(\alpha+1)\right)$. Pelo lema 3.7 de [Fa2], $\left(f_{n}^{\prime}\right)_{n \in b}$ tem supremo em $C\left(K_{\xi}\right)$ e isso prova (f).

Observe que $\int f_{n}^{\prime} d \mu_{m}=\int e_{n}^{\prime} d \mu_{m}(\alpha)$, para todo $n, m$, já que $f_{n}^{\prime}$ é determinada pelas coordenadas abaixo de $\alpha$. Logo também concluímos o item (e).

Resta-nos provar o item $(g)$. Suponha que existam $U_{1}$ e $U_{2}$ subconjuntos abertos de $K_{\eta}$ tais que $x_{n}(\alpha) \in U_{1}$, para todo $n \in b$, e $x_{n}(\alpha) \in U_{2}$, para 
todo $n \in a \backslash b$. Pela compacidade de $K_{\eta}$, podemos assumir que $U_{1}$ e $U_{2}$ são uniões finitas de abertos elementares. Portanto existe $\beta<\omega_{1}$, que pode ser escolhido maior do que $\alpha$, tal que a separação ocorre abaixo de $\beta$, i.e., $\overline{\left\{x_{n} \mid \beta: n \in b\right\}} \cap \overline{\left\{x_{n} \mid \beta: n \in a \backslash b\right\}}=\emptyset$, em $K_{\eta}(\beta)$. Como $x_{n} \mid \alpha=x_{n}(\alpha)$, te$\operatorname{mos} x_{n} \mid \beta \in F_{n}^{\alpha, \eta}(\beta)$. Pela construção temos que $L_{(\alpha, 0)}^{\beta, \eta} \backslash L_{(\alpha, 0)}^{\alpha+1, \eta}$ é finito. Como $L_{(\alpha, 0)}^{\alpha+1, \eta}=b$ e $\lim _{n \in L_{(\alpha, 0)}^{\beta, \eta}} F_{n}^{\alpha, \eta}(\beta)=z_{(\alpha, 0)}^{\beta, \eta}$, temos que $z_{(\alpha, 0)}^{\beta, \eta} \in \overline{\left\{x_{n} \mid \beta: n \in b\right\}}$. Analogamente concluímos que $z_{(\alpha, 0)}^{\beta, \eta} \in \overline{\left\{x_{n} \mid \beta: n \in a \backslash b\right\}}$, contradizendo o fato de que $\overline{\left\{x_{n} \mid \beta: n \in b\right\}} \cap \overline{\left\{x_{n} \mid \beta: n \in a \backslash b\right\}}=\emptyset$.

Agora vamos provar a parte $(i i)$ da Proposição. Para isso, seja $L$ um subconjunto fechado de $K_{\xi}$ e sejam $V_{1}$ e $V_{2}$ dois abertos de $L$ disjuntos tais que $\bar{V}_{1} \cap \bar{V}_{2} \neq \emptyset$. Tome $U$ e $V$ abertos de $[0,1]^{\omega_{1}}$ tais que $V_{1}=U \cap L$ e $V_{2}=V \cap L$. Como $L$ é fechado, $\bar{V}_{1} \cap \bar{V}_{2}=\bar{U} \cap \bar{V} \cap L$, pois $\overline{U \cap L}=\bar{U} \cap L$.

Pela separabilidade de $[0,1]^{\omega_{1}}$ (veja $\left[\right.$ Eng], 2.3.16), temos que $[0,1]^{\omega_{1}}$ satisfaz a condição da cadeia enumerável, i.e., toda família de abertos não vazios e dois a dois disjuntos é enumerável. Logo, se tomarmos $U^{\prime} \subset U$ a união de uma família maximal de abertos elementares de $U$, teremos $\overline{U^{\prime}}=\bar{U}$, e o mesmo ocorre para $V$. Portanto, podemos assumir que $U$ e $V$ são uniões enumeráveis de abertos elementares.

Sejam $\left(y_{\alpha}\right)_{\alpha<\omega_{1}}$ e $\left(z_{\alpha}\right)_{\alpha<\omega_{1}}$ sequências densas em $V_{1}$ e $V_{2}$, respectivamente. Tome $\beta<\omega_{1}$ contendo todas as coordenadas que determinam $U$ e $V$, i.e., satisfazendo $\pi^{-1}\left[\pi_{\beta}[U]\right]=U$ e $\pi^{-1}\left[\pi_{\beta}[V]\right]=V$. Pelo Lema 3.5 existe $\alpha>\beta$ tal que $i(\alpha)=1, \varphi_{1}(\alpha)=\varphi_{2}(\alpha)$ e $\xi \in \varphi_{1}(\alpha), U_{\alpha}=U, V_{\alpha}=V,\left(y_{\beta} \mid \alpha\right)_{\beta<\alpha}$ é denso em $\pi_{\alpha}\left[V_{1}\right],\left(z_{\beta} \mid \alpha\right)_{\beta<\alpha}$ é denso em $\pi_{\alpha}\left[V_{2}\right]$ e

$$
\begin{aligned}
& \left\{y_{\beta} \mid \alpha: \beta<\alpha\right\}=A_{\alpha} ; \\
& \left\{z_{\beta} \mid \alpha: \beta<\alpha\right\}=B_{\alpha} .
\end{aligned}
$$

Tome $x \in \bar{V}_{1} \cap \bar{V}_{2}$. Como $A_{\alpha}$ e $B_{\alpha}$ são densos em $\pi_{\alpha}\left[V_{1}\right]$ e $\pi_{\alpha}\left[V_{2}\right]$, respectivamente, temos que $x \mid \alpha \in \overline{\pi_{\alpha}\left[U_{\alpha}\right] \cap A_{\alpha}} \cap \overline{\pi_{\alpha}\left[V_{\alpha}\right] \cap B_{\alpha}}$. Logo $\alpha$ é um passo não trivial. Portanto $x_{n}(\alpha) \stackrel{n \in \mathbb{N}}{\longrightarrow} x \mid \alpha$ e $x_{n}(\alpha) \in \pi_{\alpha}\left[V_{1}\right]$, se $n$ é par, e $x_{n}(\alpha) \in \pi_{\alpha}\left[V_{2}\right]$, se $n$ é ímpar.

Para todo número par $n$, tome $\alpha_{n}$ tal que $y_{\alpha_{n}} \mid \alpha=x_{n}(\alpha)$. Para todo 
número ímpar $n$, tome $\alpha_{n}$ tal que $z_{\alpha_{n}} \mid \alpha=x_{n}(\alpha)$. Como na demonstração do item $(g)$ da parte $(i)$, temos

$$
\begin{aligned}
& \overline{\left\{y_{\alpha_{n}}: n \in L_{(\alpha, 0)}^{\alpha+1, \xi}\right\}} \cap \overline{\left\{z_{\alpha_{n}}: n \in R_{(\alpha, 0)}^{\alpha+1, \xi}\right\}} \neq \emptyset ; \\
& \overline{\left\{y_{\alpha_{n}}: n \in L_{(\alpha, 1)}^{\alpha+1, \xi}\right\}} \cap \overline{\left\{z_{\alpha_{n}}: n \in R_{(\alpha, 1)}^{\alpha+1, \xi}\right\}} \neq \emptyset .
\end{aligned}
$$

Sejam

$$
z_{1} \in \overline{\left\{y_{\alpha_{n}}: n \in L_{(\alpha, 0)}^{\alpha+1, \xi}\right\}} \cap \overline{\left\{z_{\alpha_{n}}: n \in R_{(\alpha, 0)}^{\alpha+1, \xi}\right\}}
$$

e

$$
z_{2} \in \overline{\left\{y_{\alpha_{n}}: n \in L_{(\alpha, 1)}^{\alpha+1, \xi}\right\}} \cap \overline{\left\{z_{\alpha_{n}}: n \in R_{(\alpha, 1)}^{\alpha+1, \xi}\right\}} .
$$

Temos então $z_{1}, z_{2} \in \bar{V}_{1} \cap \bar{V}_{2} \mathrm{e}$

$$
z_{1}\left|(\alpha+1)=z_{(\alpha, 0)}^{\alpha+1, \xi} \neq z_{(\alpha, 1)}^{\alpha+1, \xi}=z_{2}\right|(\alpha+1)
$$

Segue então que $\left|\bar{V}_{1} \cap \bar{V}_{2}\right| \geq 2$, o que encerra a prova da proposição.

Proposição 3.11. ( $\diamond)$ Seja $\xi<2^{\omega_{1}}$. Então $K_{\xi}$ é um espaço conexo $e$ hereditariamente Koszmider. Em particular, $C(L)$ é indecomponível para todo fechado conexo $L \subseteq K_{\xi}$.

Demonstração. Pela Proposição 3.10 temos que $K_{\xi}$ possui exatamente as mesmas propriedades topológicas estabelecidas pelo Teorema 5.2 de [Fa2]. A conclusão segue da demonstração do Teorema 5.3 de [Fa2].

Lembramos que um operador $T$ em um espaço de Banach $X$ é de Fredholm se a dimensão do núcleo e a codimensão da imagem são finitos (vide [DS]).

Definição 3.12. Dois espaços de Banach $X$ e $Y$ são essencialmente incomparáveis se, para todos operadores $T: X \longrightarrow Y$ e $S: Y \longrightarrow X$, o operador $I-S \circ T: X \longrightarrow X$ é um operador de Fredholm.

Lema 3.13. Sejam $K$ e $L$ espaços topológicos compactos tais que todo operador $T: C(K) \longrightarrow C(L)$ é fracamente compacto. Então $C(K)$ e $C(L)$ são essencialmente incomparáveis. 
Demonstração. Sabemos que a composição de um operador fracamente compacto com qualquer operador contínuo é um operador fracamente compacto. Por um resultado de [LT] temos que a soma de um operador de Fredholm e um operador estritamente singular é um operador de Fredholm. Pelo resultado de [Pe2], um operador fracamente compacto em $C(K)$ é estritamente singular. Note que o operador identidade é de Fredholm, já que $\operatorname{dim}(\operatorname{Ker}(I))=\operatorname{codim}(\operatorname{Im}(I))=0$. Logo, para $K$ e $L$ como na hipótese do lema, $I-S \circ T$ é um operador de Fredholm em $C(K)$, para todos $T: C(K) \longrightarrow C(L)$ e $S: C(L) \longrightarrow C(K)$.

Observação: Note que para o Lema 3.13 assumimos que todo operador é fracamente compacto apenas em uma direção, de $C(K)$ em $C(L)$, e concluímos que $C(K)$ e $C(L)$ são essencialmente incomparáveis. A existência de um operador não fracamente compacto de $C(K)$ em $C(L)$ não implica a existência de um operador não fracamente compacto de $C(L)$ em $C(K)$.

Lema 3.14. ([DU], VI, Cor. 17) Sejam $K$ um espaço compacto e $X$ um espaço de Banach. Um operador $S: C(K) \longrightarrow X$ é fracamente compacto se, e somente se, para toda sequência limitada e duas a duas disjunta $\left(f_{n}\right)_{n \in \omega}$ em $C(K)$, a sequência $\left(\left\|S\left(f_{n}\right)\right\|\right)_{n \in \omega}$ converge a 0 .

Proposição 3.15. ( $\diamond)$ Sejam $\xi, \eta<2^{\omega_{1}}$. Se $\xi \neq \eta$, então todo operador $T: C\left(K_{\xi}\right) \longrightarrow C\left(K_{\eta}\right)$ é fracamente compacto.

Demonstração. Suponha que exista $T: C\left(K_{\xi}\right) \longrightarrow C\left(K_{\eta}\right)$ não fracamente compacto, para $\xi \neq \eta$. Pelo Lema 3.14, existe sequência limitada $f_{n} \in C\left(K_{\xi}\right)$ de funções duas a duas disjuntas e $\varepsilon>0$ tais que $\left\|T\left(f_{n}\right)\right\|>\varepsilon$, para todo $n$. Considerando múltiplos de $\max \left(f_{n}, 0\right)$ e $-\min \left(f_{n}, 0\right)$ podemos assumir, sem perda de generalidade, que $f_{n}$ tem imagem contida em $[0,1]$.

Para cada $n \in \omega$ escolhemos $x_{n} \in K_{\eta}$ tal que $\left|T\left(f_{n}\right)\left(x_{n}\right)\right|>\varepsilon$. Como somas finitas de $f_{n}$ 's são uniformemente limitadas, se $x_{n}$ fosse constante para infinitos valores de $n$, teríamos uma contradição com o fato de $T$ ser limitada (isto é, contínua). Logo, podemos assumir, sem perda de generalidade, que os $x_{n}$ 's são todos distintos. Além disso, como $K_{\eta}$ é compacto, toda sequência de pontos distintos contém uma subsequência relativamente discreta e, portanto, 
podemos também assumir, sem perda de generalidade, que $\left(x_{n}\right)_{n \in \omega}$ é uma sequência relativamente discreta. Considere $\mu_{n}=T^{*}\left(\delta_{x_{n}}\right)$, para $n \in \omega$. Temos então que

$$
\left|T\left(f_{n}\right)\left(x_{n}\right)\right|=\left|\int f_{n} d \mu_{n}\right|>\varepsilon .
$$

Aplicando o Lema de Rosenthal (veja [Di,pg.82]), encontramos $N^{\prime} \subseteq \omega$ infinito tal que

$$
\Sigma\left\{\left|\int f_{m} d \mu_{n}\right|: n \neq m, m \in N^{\prime}\right\}<\frac{\varepsilon}{3} .
$$

Se aplicarmos a Proposição 3.10 para $\left\{f_{n}: n \in N^{\prime}\right\},\left\{x_{n}: n \in N^{\prime}\right\}$, $\left\{\mu_{n}: n \in N^{\prime}\right\}$ e $\varepsilon$, achamos $b \subset a \subset N^{\prime}, \delta>0$ e funções $\left\{f_{n}^{\prime}: n \in a\right\}$ tais que :

- $\operatorname{supp}\left(f_{n}^{\prime}\right) \subset \operatorname{supp}\left(f_{n}\right)$;

- $\left|\int f_{n}^{\prime} d \mu_{n}\right|>\delta$ e $\Sigma\left\{\int f_{m}^{\prime} d\left|\mu_{n}\right|: m \neq n, m \in a\right\}<\delta / 3$, para todo $n \in a$;

- $\left(f_{n}^{\prime}\right)_{n \in b}$ tem supremo em $C\left(K_{\xi}\right)$;

- $\overline{\left\{x_{n}: n \in b\right\}} \cap \overline{\left\{x_{n}: n \in a \backslash b\right\}} \neq \emptyset$.

Podemos assumir que

$$
\int \sup \left\{f_{m}^{\prime}: m \in b\right\} d \mu_{n}=\int \Sigma_{m \in b} f_{m}^{\prime} d \mu_{n}
$$

para todo $n \in \omega$. Com efeito, tome $\left(N_{\xi}\right)_{\xi<\omega_{1}}$ uma família de subconjuntos infinitos de $N^{\prime}$ tais que $N_{\xi} \cap N_{\eta}$ é finito, para todo $\xi \neq \eta$ (podemos construir tal família identificando $\mathbb{N} \operatorname{com} \mathbb{Q}$ e $\omega_{1} \operatorname{com} \mathbb{R}$, e tomando $N_{\xi}$ uma sequência racional convergindo para $\xi$ ). Para todo $\xi$ tome $b_{\xi} \subset a_{\xi} \subset N_{\xi}$ como $a$ e $b$ da Proposição 3.10 .

Para todos $\xi<\omega_{1}$ e $n \in b_{\xi}$ considere $f_{n}^{\xi}$ como as $f_{n}^{\prime}$ da Proposição 3.10, i.e., as propriedades $(e),(f)$ e $(g)$ da proposição 3.10 acontecem para $f_{n}^{\prime}=f_{n}^{\xi}$, $a=a_{\xi}$ e $b=b_{\xi}$.

Afirmação 3.15.1. Existe $\xi<\omega_{1}$ tal que

$$
\int\left[\sup \left\{f_{m}^{\xi}: m \in b_{\xi}\right\}-\Sigma_{m \in b_{\xi}} f_{m}^{\xi}\right] d \mu_{n}=0,
$$


para todo $n \in \mathbb{N}$.

Demonstração: Para todo $\xi<\omega_{1}$ e todo $c \subset b_{\xi}$ definiremos a função $f_{c}^{\xi}=\sup \left\{f_{m}^{\xi}: m \in c\right\}-\Sigma_{m \in c} f_{m}^{\xi}$ sempre que o supremo existir. Observe que, para todo subconjunto finito $F \subset b_{\xi}$, temos

$$
\sup \left\{f_{m}^{\xi}: m \in b_{\xi}\right\}=\sup \left\{f_{m}^{\xi}: m \in b_{\xi} \backslash F\right\}+\Sigma_{m \in F} f_{m}^{\xi}
$$

e, portanto, $f_{b_{\xi} \backslash F}^{\xi}=f_{b_{\xi}}^{\xi}$. Em particular, $f_{b_{\xi}}^{\xi}=f_{b_{\xi} \backslash b_{\xi^{\prime}}}^{\xi}$, para todos $\xi \neq \xi^{\prime} \mathrm{em}$ $\omega_{1}$, já que $b_{\xi} \cap b_{\xi^{\prime}}$ é finito.

Sejam $\xi$ e $\xi^{\prime}$ ordinais distintos em $\omega_{1}$. Tome $g=\sup \left\{f_{n}^{\xi}: n \in b_{\xi} \backslash b_{\xi^{\prime}}\right\}$ e $h=\sup \left\{f_{n}^{\xi^{\prime}}: n \in b_{\xi^{\prime}} \backslash b_{\xi}\right\}$. Como $\operatorname{supp}\left(f_{n}^{\eta}\right) \subset \operatorname{supp}\left(f_{n}\right)$, para todo $n \in \mathbb{N}$ e $\eta<\omega_{1}$, temos que $f_{n}^{\xi} \cdot f_{m}^{\xi^{\prime}}=0$, para todo $n \neq m$. Vamos agora provar que $g \cdot h=0$.

Suponha que exista $x \in K$ tal que $g(x)>0$ e $h(x)>0$. Então existe uma vizinhança aberta $V$ de $x$ tal que as restrições de $g$ e $h$ em $V$ são ambas estritamente positivas. Logo, existem $y \in V$ e $n \in b_{\xi} \backslash b_{\xi^{\prime}}$ tais que $f_{n}^{\xi}(y)>0$. Tome $\varphi$ uma função contínua de $K$ em $[0,1]$ tal que $\varphi(y)=1$ e $\varphi$ é nula nos pontos em que $f_{n}^{\xi}$ é nula. Como $f_{n}^{\xi} \cdot f_{m}^{\xi^{\prime}}=0$, para todo $m \in b_{\xi^{\prime}} \backslash b_{\xi}$, temos que $f_{m}^{\xi^{\prime}} \leq h \cdot \varphi<h$, para todo $m \in b_{\xi^{\prime}} \backslash b_{\xi}$, contradizendo a definição de $h$.

Como $f_{b_{\xi}}^{\xi}=f_{b_{\xi} \backslash b_{\xi^{\prime}}}^{\xi} \leq g$ e $f_{b_{\xi^{\prime}}}^{\xi^{\prime}}=f_{b_{\xi^{\prime}} \backslash b_{\xi}}^{\xi^{\prime}} \leq h$, segue que $f_{b_{\xi}}^{\xi} \cdot f_{b_{\xi^{\prime}}}^{\xi^{\prime}}=0$, para todo $\xi \neq \xi^{\prime}$. Portanto existe $\xi<\omega_{1}$ tal que

$$
\int f_{b_{\xi}}^{\xi} d \mu_{n}=0
$$

para todo $n$, o que encerra a prova da Afirmação.

Tomando $f=\sup \left\{f_{n}^{\prime}: n \in b\right\}$ e $n \in b$ temos

$$
\begin{aligned}
\left|T(f)\left(x_{n}\right)\right|=\left|\int f d \mu_{n}\right| & =\left|\int f_{n}^{\prime} d \mu_{n}+\int \Sigma\left\{f_{m}^{\prime}: m \neq n, m \in b\right\} d \mu_{n}\right| \\
& \geq \delta-\delta / 3=2 \delta / 3 .
\end{aligned}
$$


Por outro lado, se $n \in a \backslash b$ temos

$$
\left|T(f)\left(x_{n}\right)\right|=\left|\int \Sigma_{m \in b} f_{m}^{\prime} d \mu_{n}\right| \leq \delta / 3
$$

Contradizendo que $T(f)$ é contínua e o fato de que

$$
\overline{\left\{x_{n}: n \in b\right\}} \cap \overline{\left\{x_{n}: n \in a \backslash b\right\}} \neq \emptyset .
$$

Unindo a construção e as Proposições 3.11 e 3.15, obtemos o resultado desejado:

Teorema 3.16. $(\diamond)$ Existe uma família $\left(K_{\xi}\right)_{\xi<2^{\left(2^{\omega}\right)}}$ de espaços conexos e hereditariamente Koszmider tais que todo operador de $C\left(K_{\xi}\right)$ em $C\left(K_{\eta}\right)$ é fracamente compacto, para $\xi \neq \eta$. Em particular, $\left(C\left(K_{\xi}\right)\right)_{\xi<2^{\left(2^{2}\right)}}$ é uma família de espaços de Banach indecomponíveis e dois a dois essencialmente incomparáveis. 


\section{Capítulo 4}

\section{Construção alternativa de um espaço hereditariamente fracamente Koszmider}

Assumindo o princípio $\diamond$, Fajardo constrói, em [Fa2], o primeiro exemplo relativamente consistente com ZFC de um espaço de Banach indecomponível $C(K)$ tal que, para todo fechado $L \subseteq K$, todo operador em $C(L)$ é multiplicação fraca. O objetivo deste capítulo é apresentar um método alternativo de construção de espaços $C(K)$ com poucos operadores satisfazendo a propriedade de que $C(L)$ também tem poucos operadores, para todo fechado $L \subseteq K$. O método que utilizaremos será o método do forcing e o espaço compacto $K$ que obteremos será conexo e hereditariamente fracamente Koszmider, isto é, para todo fechado $L \subseteq K$, todo operador em $C(L)$ é multiplicador fraco. Em particular, o compacto $K$ responde positivamente ao problema de Efimov. O método do forcing foi criado por Paul Cohen, em 1964, para se mostrar a consistência da negação da hipótese do contínuo, resultado que lhe rendeu a medalha Fields, em 1966. No final do capítulo apresentamos um resultado envolvendo a densidade dos espaços $C(K)$ com poucos operadores. 


\subsection{A construção do forcing}

Vamos iniciar nossa construção do forcing assumindo que no ground model $V$ vale $C H$ e que $\omega_{2}=2^{\omega_{1}}$. Seja $\kappa=\omega_{2}$.

Se $J \subseteq I \subseteq \kappa$, denotaremos por $\pi_{I, J}$ a projeção usual de $[0,1]^{I}$ sobre $[0,1]^{J}$, dada por $\pi_{I, J}(x)=\left.x\right|_{J}$. Quando $I$ estiver claro pelo contexto especialmente quando $I=\kappa$ - denotaremos $\pi_{I, J}$ simplesmente por $\pi_{J}$.

Seja a um subconjunto infinito de $\mathbb{N}$ e seja $\left(F_{n}\right)_{n \in a}$ uma sequência de subconjuntos fechados de um espaço topológico $K$. Dizemos que $\left(F_{n}\right)_{n \in a}$ converge para $x \in K$ se o conjunto $\left\{n \in a: F_{n} \nsubseteq \amalg U\right.$ é finito, para toda vizinhança aberta $U$ of $x$.

Definimos então a noção de forcing $\langle\mathbb{P}, \leq\rangle$ tomando $\mathbb{P}$ o conjunto de todos os elementos $p=\left(I_{p}, K_{p}, \mathcal{P}_{p}, \mathcal{B}_{p}\right)$ satisfazendo as seguintes condições:

P1 $I_{p} \subseteq \kappa \mathrm{e}\left|I_{p}\right| \leq \omega$

P2 $K_{p} \subseteq[0,1]^{I_{p}}$ é compacto e conexo;

P3 $\mathcal{P}_{p}$ é um conjunto enumerável de 4 -uplas $\left(\left(F_{n}\right)_{n \in \mathbb{N}}, a, b, z\right)$ tais que

- $\left(F_{n}\right)_{n \in \mathbb{N}}$ é uma sequência de subconjuntos dois a dois disjuntos e fechados de $K_{p}$;

- $a, b \subseteq \mathbb{N}$ são subconjuntos infinitos e disjuntos;

- $z \in K_{p}$;

- $\left(F_{n}\right)_{n \in a}$ e $\left(F_{n}\right)_{n \in b}$ convergem para $z$.

$\mathbf{P} 4 \mathcal{B}_{p}=\left\{\left(x_{\xi}^{p}, y_{\xi}^{p}\right): \xi \in I_{p}\right\}$, onde $x_{\xi}^{p}, y_{\xi}^{p} \in K_{p}$ e $\left.x_{\xi}^{p}\right|_{I_{p} \cap \xi}=\left.y_{\xi}^{p}\right|_{I_{p} \cap \xi}$.

Definimos também a relação de ordem $\leq$ dizendo que $q \leq p$ se, e somente se,

O1 $I_{q} \supseteq I_{p}$

O2 $\pi_{I_{q}, I_{p}}\left[K_{q}\right]=K_{p}$

O3 Se $M$ é raro em $K_{p}$, então $\pi_{I_{q}, I_{p}}^{-1}[M]$ é raro em $K_{q}$; 
O4 Para todo $\left(\left(F_{n}\right)_{n \in \mathbb{N}}, a, b, z\right) \in \mathcal{P}_{p}$ existe $\left(\left(F_{n}^{\prime}\right)_{n \in \mathbb{N}}, a^{\prime}, b^{\prime}, z^{\prime}\right) \in \mathcal{P}_{q}$ tal que

- $b^{\prime} \backslash b$ e $a^{\prime} \backslash a$ são conjuntos finitos;

- $\left.z^{\prime}\right|_{I_{p}}=z$;

- $F_{n}^{\prime}=\pi_{I_{q}, I_{p}}^{-1}\left[F_{n}\right]$, para todo $n \in \mathbb{N}$.

O5 Para todo $\xi \in I_{p}$ temos $\left.x_{\xi}^{q}\right|_{I_{p}}=x_{\xi}^{p}$ e $\left.y_{\xi}^{q}\right|_{I_{p}}=y_{\xi}^{p}$.

Lema 4.1. ( $\mathbb{P}, \leq)$ é $\sigma$-fechado. I.e., se $\left(p_{n}\right)_{n \in \mathbb{N}}$ é uma sequência em $\mathbb{P}$ tal que $p_{n+1} \leq p_{n}$, para todo $n \in \mathbb{N}$, então existe $p \in \mathbb{P}$ tal que $p \leq p_{n}$, para todo $n \in \mathbb{N}$.

Demonstração. Seja $\left(p_{n}\right)_{n \in \mathbb{N}}$ uma sequência como na hipótese do lema. Precisamos encontrar $p=\left(I_{p}, K_{p}, \mathcal{P}_{p}, \mathcal{B}_{p}\right)$ tal que $p \in \mathbb{P}$ e $p \leq p_{n}$, para todo $n \in \mathbb{N}$.

Tome $I_{p}=\bigcup_{n \in \mathbb{N}} I_{p_{n}}, K_{p}$ o limite inverso de $\left(K_{p_{n}}\right)_{n \in \mathbb{N}}$ e $\mathcal{B}_{p}=\left\{\left(x_{\xi}^{p}, y_{\xi}^{p}\right)\right.$ : $\left.\xi \in I_{p}\right\}$, onde $x_{\xi}^{p}=\bigcup_{n \geq n_{0}} x_{\xi}^{p_{n}}, y_{\xi}^{p}=\bigcup_{n \geq n_{0}} y_{\xi}^{p_{n}}$ e $n_{0}$ é o menor inteiro tal que $\xi \in I_{p_{n_{0}}}$. Claramente $I_{p}$ e $\mathcal{B}_{p}$ satisfazem as condições P1 e P4. Além disso, como o limite inverso preserva compacidade e conexidade, $K_{p}$ satisfaz P2.

Resta-nos agora definir $\mathcal{P}_{p}$. Para isso, construiremos uma função $\phi$ cujo domínio é $\bigcup \mathcal{P}_{p_{n}}$ e tomaremos $\mathcal{P}_{p}$ como sendo a imagem de $\phi$.

Seja $Q \in \mathcal{P}_{n_{0}}$, para algum $n_{0}$. Fixe uma sequência $\left(\left(F_{m}^{n}\right)_{m \in \mathbb{N}}, a_{n}, b_{n}, z_{n}\right)_{n \geq n_{0}}$ tal que $Q=\left(\left(F_{m}^{n_{0}}\right)_{m \in \mathbb{N}}, a_{n_{0}}, b_{n_{0}}, z_{n_{0}}\right)$ e, para cada natural $n>n_{0}$, a 4-upla $\left(\left(F_{m}^{n}\right)_{m \in \mathbb{N}}, a_{n}, b_{n}, z_{n}\right) \in \mathcal{P}_{p_{n}}$ é obtida a partir de $\left(\left(F_{m}^{n-1}\right)_{m \in \mathbb{N}}, a_{n-1}, b_{n-1}, z_{n-1}\right)$ utilizando a condição $\mathrm{O} 4$ da relação de ordem do forcing.

Para todo $m \in \mathbb{N}$, tome $F_{m}$ o limite inverso de $\left(F_{m}^{n}\right)_{n \geq n_{0}}$. Seja a uma pseudo-intersecção infinita de $\left(a_{n}\right)_{n \geq n_{0}}$, i.e., $a \backslash a_{n}$ é um conjunto finito, para todo $n \geq n_{0}$ (a existência de tal pseudo-intersecção segue do fato de que $a_{n+1} \backslash a_{n}$ é finito. Veja [Do], Teorema 3.1).

Tome $b^{\prime}$ uma pseudo-intersecção infinita de $\left(b_{n}\right)_{n \geq n_{0}}$ e defina $b=b^{\prime} \backslash a$. Está claro que $a \cap b^{\prime}$ é finito e, portanto, $b$ continua sendo uma pseudointersecção infinita de $\left(b_{n}\right)_{n \geq n_{0}}$.

Finalmente, tome $z=\bigcup_{n \geq n_{0}} z_{n}$ e defina $\phi(Q)=\left(\left(F_{m}\right)_{m \in \mathbb{N}}, a, b, z\right)$. Vamos agora provar P3. O fato de $a$ e $b$ serem disjuntos segue imediatamente da 
definição. Claramente $z \in K_{p}$ e $\left(F_{m}\right)_{m \in \mathbb{N}}$ é uma sequência de subconjuntos fechados de $K_{p}$ e dois a dois disjuntos. Suponha que $\left(F_{m}\right)_{m \in a}$ não convirja para $z$. Isto significa que existem uma vizinhança aberta básica $U$ de $z$, um subconjunto infinito $c \subset a$ e uma sequência $\left(x_{m}\right)_{m \in c}$ tais que $x_{m} \in F_{m} \backslash U$, para todo $m \in c$. Tomando $n \in \mathbb{N}$ tal que $U$ depende apenas de coordenadas que estão em $I_{p_{n}}$, temos que $\pi_{I_{p_{n}}}[U]$ é uma vizinhança aberta de $z_{n}$, em $K_{p_{n}}$, e $x_{m} \mid I_{p_{n}} \in F_{m}^{n} \backslash \pi_{I_{p_{n}}}[U]$, para todo $m \in c$. Como $a \backslash a_{n}$ é finito, segue que $a_{n} \cap c$ é um subconjunto infinito de $a_{n}$, contradizendo a condição P3 em $p_{n}$. A prova de que $\left(F_{m}\right)_{m \in b}$ converge para $z$ é a mesma.

Logo, tomando $\mathcal{P}_{p}$ a imagem de $\phi$, concluímos a construção de $p$ e já provamos que $p \in \mathbb{P}$. Ainda nos falta provar que $p \leq p_{n}$, para todo $n \in \mathbb{N}$. As condições O1, O2 e O5 são facilmente verificadas, e O4 segue da definição da função $\phi$. Para verificar O3, seja $M$ raro em $K_{p_{n}}$, para $\operatorname{algum} n$, e suponha que $\pi_{I_{p}, I_{p_{n}}}^{-1}[M]$ não seja raro em $K_{p}$. Seja $V \subseteq \overline{\pi_{I_{p}, I_{p_{n}}}^{-1}[M]}$ um aberto não-vazio. Tome $m>n$ tal que as coordenadas que determinam $V$ estão em $I_{p_{m}}$. Note que $\pi_{I_{p_{m}}, I_{p_{n}}}^{-1}[\bar{M}]$ é raro em $K_{p_{m}}$ pois $p_{m} \leq p_{n}$ e $\bar{M}$ é raro em $K_{p_{n}}$. Como $\overline{\pi_{I_{p}, I_{p_{n}}}^{-1}[M]} \subseteq \pi_{I_{p}, I_{p_{n}}}^{-1}[\bar{M}]$ temos

$$
\begin{gathered}
\pi_{I_{p}, I_{p_{m}}}[V] \subseteq \pi_{I_{p}, I_{p_{m}}}\left[\pi_{I_{p}, I_{p_{n}}}^{-1}[\bar{M}]\right]=\pi_{I_{p}, I_{p_{m}}}\left[\pi_{I_{p}, I_{p_{m}}}^{-1}\left[\pi_{I_{p_{m}}, I_{p_{n}}}^{-1}[\bar{M}]\right]\right]=\pi_{I_{p_{m}}, I_{p_{n}}}^{-1}[\bar{M}] \\
\text { e } \pi_{I_{p}, I_{p_{m}}}[V] \text { é aberto em } K_{p_{m}}, \text { contradizendo que } \pi_{I_{p_{m}, I_{p_{n}}}^{-1}}^{-1}[\bar{M}] \text { é raro em } K_{p_{m}} .
\end{gathered}
$$

Lema 4.2. Seja $p \in \mathbb{P}$. Dados

(a) uma sequência $\left(f_{n}: n \in \mathbb{N}\right)$ de funções contínuas e duas a duas disjuntas de $K_{p}$ em $[0,1]$;

(b) uma sequência relativamente discreta $\left(x_{n}\right)_{n \in \mathbb{N}}$ de pontos distintos de $K_{p}$ tais que $x_{n} \notin \operatorname{supp}\left(f_{m}\right)$, para todos $n, m \in \mathbb{N}$;

(c) $u m \varepsilon>0$;

(d) uma sequência limitada $\left(\mu_{n}: n \in \mathbb{N}\right)$ de medidas regulares sobre $K$ tais que $\left|\int f_{n} d \mu_{n}\right|>\varepsilon$, para todo $n \in \mathbb{N}$; 
existem $q \leq p, \delta>0, b \subset a \subset \mathbb{N}$ subconjuntos infinitos, $z^{\prime} \in K_{q}$ e funções contínuas $f_{n}^{\prime}: K_{p} \longrightarrow[0,1]$ tais que $\operatorname{supp}\left(f_{n}^{\prime}\right) \subset \operatorname{supp}\left(f_{n}\right) e$

(e) $\left|\int f_{n}^{\prime} d \mu_{n}\right|>\delta$ e $\Sigma\left\{\int f_{m}^{\prime} d\left|\mu_{n}\right|: m \neq n, m \in a\right\}<\delta / 3$, para todo $n \in a$;

(f) $K_{q}=K_{p}\left(\left(f_{n}^{\prime}\right)_{n \in b}\right)$ é uma extensão completa;

(g) $\left(f_{n}^{\prime} \circ \pi_{I_{q}, I_{p}}\right)_{n \in b}$ tem supremo em $C\left(K_{q}\right)$;

(h) $\left(\left(\pi_{I_{q}, I_{p}}^{-1}\left[\left\{x_{n}\right\}\right]\right)_{n \in \mathbb{N}}, b, a \backslash b, z^{\prime}\right) \in \mathcal{P}_{q}$.

Demonstração. O resultado segue imediatamente da Proposição 3.8 e da definição de $\mathbb{P}$, tomando $I_{q}=I_{p} \cup\{\alpha\}$, onde $\alpha$ é qualquer limitante superior de $I_{p}$ em $\kappa$. A única questão adicional a qual devemos nos preocupar é definir $\mathcal{B}_{q}$ e provar 05 . Mas isso segue facilmente, já que podemos tomar $x_{\xi}^{q}$ e $y_{\xi}^{q}$ quaisquer extensões de $x_{\xi}^{p}$ e $y_{\xi}^{p}$, respectivamente, em $K_{q}$, para $\xi \in I_{p}$, e $x_{\alpha}^{q}=y_{\alpha}^{q}$ qualquer elemento de $K_{q}$. Como $\alpha>\xi$, para todo $\xi \in I_{p}$, temos que O5 é preservada em $q$.

Lema 4.3. Dados $p \in \mathbb{P}$ e $\alpha<\kappa$, existem $q \leq p$ e $\xi>\alpha$ tais que $\xi \in I_{q} e$ $x_{\xi}^{q} \neq y_{\xi}^{q}$.

Demonstração. Fixe $z \in K_{p}$ e $\left(z_{n}\right)_{n \in \mathbb{N}}$ uma sequência em $K_{p}$ convergindo para $z$. Escolha vizinhanças abertas $V_{n}$ de $z_{n}$ duas a duas disjuntas e cujos diâmetros tendem a zero. Assuma, também, que $z \notin V_{n}$, para todo $n$. Fixe funções contínuas $f_{n}: K_{p} \longrightarrow[0,1]$ tais que $f_{n}\left(z_{n}\right)=1$ e $\operatorname{supp}\left(f_{n}\right) \subset V_{n}$. Defina $\mu_{n}=\delta_{z_{n}}$ e $\varepsilon=\frac{1}{2}$. Tome $\left(x_{n}\right)_{n \in \mathbb{N}}$ qualquer sequência relativamente discreta em $K_{p}$.

Tome $\xi>\max \left\{\alpha, \operatorname{supI}_{p}\right\}$ em $\kappa$ e $q \leq p$ como no Lema 4.2, considerando $I_{q}=I_{p} \cup\{\xi\}$. Pelo item $(e)$ desse lema temos que $f_{n}^{\prime}\left(z_{n}\right)>\delta$. Logo, tomando $t$ ponto de acumulação de $\left\{f_{n}^{\prime}\left(z_{n}\right): n \in \mathbb{N}\right\}$ temos que $t \geq \delta$ e $(z, t) \in K_{q}$. Defina $x_{\xi}^{q}=(z, 0)$ e $y_{\xi}^{q}=(z, t)$.

Lema 4.4. Seja $p \in \mathbb{P}$. Tome $\left(f_{n}\right)_{n \in \mathbb{N}}$ uma sequência de funções contínuas e duas a duas disjuntas de $K_{p}$ em $[0,1]$ e suponha que $\left(f_{n}\right)_{n \in \mathbb{N}}$ tem supremo $f$ em $C\left(K_{p}\right)$. Então, para todo $q \leq p, f \circ \pi_{I_{q}, I_{p}}$ é o supremo de $\left(f_{n} \circ \pi_{I_{q}, I_{p}}\right)_{n \in \mathbb{N}}$ em $C\left(K_{q}\right)$. 
Demonstração. Pelo Lema 2.3, $f$ é o supremo de $\left(f_{n}\right)_{n \in \mathbb{N}}$ em $C\left(K_{p}\right)$ se, e somente se, o conjunto

$$
\Delta\left(f,\left(f_{n}\right)_{n \in \mathbb{N}}\right)=\left\{x \in K_{p}: \Sigma_{n \in \mathbb{N}} f_{n}(x) \neq f(x)\right\}
$$

é raro em $K_{p}$.

Como $\Delta\left(f \circ \pi_{I_{q}, I_{p}},\left(f_{n} \circ \pi_{I_{q}, I_{p}}\right)_{n \in \mathbb{N}}\right)=\pi_{I_{q}, I_{p}}^{-1}\left[\Delta\left(f,\left(f_{n}\right)_{n \in \mathbb{N}}\right)\right]$, pela condição P3 e pelo Lema 4.3 de [Ko2] segue que $\Delta\left(f \circ \pi_{I_{q}, I_{p}},\left(f_{n} \circ \pi_{I_{q}, I_{p}}\right)_{n \in \mathbb{N}}\right)$ é raro em $K_{q}$, provando que $f \circ \pi_{I_{q}, I_{p}}$ é o supremo de $f_{n} \circ \pi_{I_{q}, I_{p}}$ em $C\left(K_{q}\right)$.

\subsection{Extensão Genérica}

Como $\mathbb{P}$ é $\sigma$-fechado sabemos que nossa noção de forcing não adiciona conjuntos enumeráveis (veja $[\mathrm{Ku}]$ ). Em particular, $\mathbb{P}$ não adiciona novos reais e, portanto, $[0,1]^{V}=[0,1]^{V[G]}$.

Seja $G$ um $\mathbb{P}$-genérico sobre $V$. Em $V[G]$, defina $I_{G}=\cup\left\{I_{p}: p \in G\right\}$. Seja $\dot{I}_{G}$ um $\mathbb{P}$-nome para $I_{G}$ em $V$.

Lema 4.5. $I_{G}$ é ilimitado em $(\kappa)^{V[G]}$.

Demonstração. Sejam $p \in \mathbb{P}$ e $\alpha<\kappa$. Como $G$ intersecta todo conjunto denso em $\mathbb{P}$, precisamos provar que existem $q \leq p$ e $\xi>\alpha$ tais que $\xi \in I_{q}$, o que segue do Lema 4.3.

O próximo lema nos diz que se $\mathbb{P}$ é $\omega_{2}$-c.c então $\left(2^{\omega_{1}}\right)^{V[G]}=\left(2^{\omega_{1}}\right)^{V}$. Observe que se $\mathbb{P}$ for $\omega_{2}$-c.c então $\mathbb{P}$ preservará cardinais, pois todo forcing $\sigma$-fechado e $\omega_{2}$-c.c preserva cardinais (veja $[\mathrm{Ku}]$ ).

Lema 4.6. Se $\mathbb{P}$ é $\omega_{2}$-c.c então $\left(2^{\omega_{1}}\right)^{V[G]}=\left(2^{\omega_{1}}\right)^{V}$.

Demonstração. Como $\mathbb{P}$ preserva cardinais, precisamos mostrar que $\left(2^{\omega_{1}}\right)^{V[G]}=$ $\kappa$. Seja, pois, $X$ um subconjunto de $\omega_{1}$ em $V[G]$. Existe nice name $\sigma$ para $X$, i.e.,

$$
\sigma=\cup_{\xi \in \omega_{1}}\{\check{\xi}\} \times A_{\xi}
$$

onde cada $A_{\xi}$ é uma anticadeia em $\mathbb{P}$. Como $\mathbb{P}$ é $\omega_{2}$-c.c temos $\left|A_{\xi}\right| \leq \omega_{1}$. Usando $C H$ no modelo inicial verifica-se que $|\mathbb{P}|=\kappa=2^{\omega_{1}}$. Logo, existem 
$\omega_{1} \times\left(2^{\omega_{1}}\right)^{\omega_{1}}=2^{\omega_{1}}=\kappa$ nice names para subconjuntos de $\omega_{1}$, o que prova que $2^{\omega_{1}}=\kappa$ em $V[G]$.

Lema 4.7. Para todo $p \in \mathbb{P}, C\left(K_{p}\right)^{V[G]}=C\left(K_{p}\right)^{V}$ e $M\left(K_{p}\right)^{V[G]}=M\left(K_{p}\right)^{V}$, onde $M\left(K_{p}\right)$ é o conjunto das medidas de Radon sobre $K_{p}$.

Demonstração. Sabemos que $P$ não adiciona subconjuntos enumeráveis de conjuntos que estão no modelo inicial e portanto temos que $[0,1]^{V[G]}=[0,1]^{V}$. Tome $f: K_{p} \longrightarrow \mathbb{R}$ uma função contínua, em $V[G]$. Vamos mostrar que $f \in V$ e que $f$ é contínua em $V$. Como $K_{p} \subseteq[0,1]^{I_{p}}$, temos que $K_{p}$ é metrizável e separável. Seja $E \subseteq K_{p}$ um subconjunto enumerável denso e considere $g=\left.f\right|_{E}$. Como $g$ é um subconjunto enumerável de $K_{p} \times \mathbb{R}$, segue que $g \in V$.

Para cada $x \in K_{p}$, como $K_{p}$ é metrizável e $E$ é denso, encontramos uma sequência $\left(x_{n}\right)_{n \in \mathbb{N}}$ em $E$ que converge para $x$, em $V$. Como convergir para um ponto é absoluto, temos que $x_{n}$ converge para $x$ em $V[G]$. Logo, como $f$ é contínua em $V[G]$, segue que $f\left(x_{n}\right)$ converge para $f(x)$. Logo $g\left(x_{n}\right)=f\left(x_{n}\right)$ também converge para $f(x)$ em $V$. Portanto podemos definir uma função $h: K_{p} \longrightarrow \mathbb{R}$ em $V$ dada por

$$
h(x)=\lim _{n \rightarrow \infty} g\left(x_{n}\right)
$$

onde $\left(x_{n}\right)_{n \in \mathbb{N}}$ é uma sequência em $E$ que converge para $x$. Pelas observações anteriores, o limite acima existe, não depende da escolha de $\left(x_{n}\right)_{n \in \mathbb{N}}, h$ é contínua em $V$ e $h(x)=f(x)$, para todo $x$, concluindo que $f \in C\left(K_{p}\right)^{V}$. O que acabamos de mostrar foi que $C\left(K_{p}\right)^{V[G]} \subseteq C\left(K_{p}\right)^{V}$. Para a outra direção basta ver que, analogamente como vimos acima, uma função em $K_{p}$ contínua em $V$ também será contínua em $V[G]$. Por fim, como medidas de Radon sobre $K_{p}$ podem ser identificadas como funções de uma base enumerável em $\mathbb{R}$, a prova de que $M\left(K_{p}\right)^{V[G]}=M\left(K_{p}\right)^{V}$ é análoga.

Lema 4.8. Se $p \Vdash\left(\dot{I} \subseteq \dot{I}_{G}\right) \wedge(|\dot{I}| \leq \check{\omega})$, então existe $q \leq p$ tal que $q \Vdash \dot{I} \subseteq \check{I}_{q}$. Demonstração. Sejam $\dot{\alpha}_{n}$ nomes para elementos de $\kappa$ tais que

$$
p \Vdash \dot{I}=\left\{\dot{\alpha}_{n}: n \in \check{\mathbb{N}}\right\}
$$


Tome $p_{-1}=p$. Supondo construído $p_{n-1}$, para $n \geq 0$, tome $p_{n}^{\prime}, q_{n} \in \mathbb{P}$ e $\alpha_{n} \in \kappa$ tais que $p_{n}^{\prime} \leq p_{n-1} \mathrm{e}$

$$
p_{n}^{\prime} \Vdash \dot{\alpha}_{n}=\check{\alpha}_{n}, \check{\alpha}_{n} \in \check{I}_{q_{n}}, \check{q}_{n} \in \dot{G} \text {. }
$$

Como $p_{n}^{\prime} \Vdash \check{p}_{n}^{\prime}, \check{q}_{n} \in \dot{G}$, achamos $p_{n}^{\prime \prime} \leq p_{n}^{\prime}$ e $p_{n} \in \mathbb{P}$ tais que

$$
p_{n}^{\prime \prime} \Vdash \check{p}_{n} \leq \check{p}_{n}^{\prime}, \check{q}_{n}
$$

Como a relação de ordem em $\mathbb{P}$ é absoluta temos

$$
p_{n} \Vdash \check{p}_{n} \leq \check{p}_{n}^{\prime}, \check{q}_{n}
$$

$\operatorname{Logo} p_{n} \Vdash \check{I}_{q_{n}} \subseteq \check{I}_{p_{n}} \mathrm{e}$

$$
p_{n} \Vdash \dot{\alpha}_{n}=\check{\alpha}_{n}, \check{\alpha}_{n} \in \check{I}_{p_{n}}
$$

Como $\left(p_{n}\right)_{n \in \mathbb{N}}$ é decrescente e $\mathbb{P}$ é $\sigma$-fechado, existe $q \in \mathbb{P}$ tal que $q \leq p_{n}$, para todo $n \in \mathbb{N}$. Portanto

$$
q \Vdash \forall n \in \check{\mathbb{N}} \dot{\alpha}_{n}=\check{\alpha}_{n}, \check{\alpha}_{n} \in \check{I}_{q} .
$$

Isto é, $q \Vdash \dot{I} \subseteq \check{I}_{q}$, como queríamos.

Em $V[G]$, definimos

$$
K=\left\{x \in[0,1]^{I_{G}}: \forall p \in G\left(\left.x\right|_{I_{p}} \in K_{p}\right)\right\}
$$

como um subespaço de $[0,1]^{I_{G}}$ com a topologia produto.

Sejam $\dot{K}$ e $\dot{G} \mathbb{P}$-nomes para $K$ e $G$, respectivamente.

Teorema 4.9. Em $V[G]$, seja $K$ como acima.

(A) $K$ é compacto e conexo;

(B) Dados 
(a) uma sequência de funções contínuas $\left(f_{n}: n \in \mathbb{N}\right)$ e duas a duas disjuntas de $K$ em $[0,1]$;

(b) uma sequência relativamente discreta $\left(x_{n}: n \in \mathbb{N}\right)$ de pontos de $K$ tais que $f_{n}\left(x_{m}\right)=0$, para todo $n, m \in \mathbb{N}$;

(c) $u m \varepsilon>0$;

(d) uma sequência limitada $\left(\mu_{n}\right)_{n \in \mathbb{N}}$ de medidas regulares sobre $K$ tais que $\left|\int f_{n} d \mu_{n}\right|>\varepsilon$, para todo $n \in \mathbb{N}$;

existem $\delta>0, b \subseteq a \subseteq \mathbb{N}$ subconjuntos infinitos e funções contínuas $f_{n}^{\prime}$ de $K$ em $[0,1]$ tais que

(e) $\operatorname{supp}\left(f_{n}^{\prime}\right) \subseteq \operatorname{supp}\left(f_{n}\right)$, para todo $n \in \mathbb{N}$;

(f) $\left|\int f_{n}^{\prime} d \mu_{n}\right|>\delta$ e $\Sigma\left\{\int f_{m}^{\prime} d\left|\mu_{n}\right|: m \neq n, m \in a\right\}<\delta / 3$, para todo $n \in a$;

(g) $\left\{f_{n}^{\prime}: n \in b\right\}$ tem supremo em $C(K)$;

(h) $\overline{\left\{x_{n}: n \in b\right\}} \cap \overline{\left\{x_{n}: n \in a \backslash b\right\}} \neq \emptyset$.

(C) Para todo fechado $L \subseteq K$, todo operador em $C(L)$ é multiplicador fraco;

(D) Se $\mathbb{P}$ preserva cardinais então $K$ tem peso $\kappa$.

Demonstração. Para $(A)$, note que $K=\bigcap_{p \in G} \pi_{I_{G}, I_{p}}^{-1}\left[K_{p}\right]$ e, portanto, como cada $K_{p}$ é compacto, temos que $K$ é fechado em $[0,1]^{I_{G}}$ e, com maior razão, compacto. Se $K$ não fosse conexo, obteríamos $V$ e $W$ abertos disjuntos de $K$ tais que $K \backslash V \cup W=\emptyset$. Como os suportes de $V$ e $W$ são finitos, achamos $p \in G$ tal que $I_{p}$ contém os suportes de $V$ e $W$, e teríamos que $\pi_{I_{p}}[V]$ e $\pi_{I_{p}}[W]$ seriam dois abertos disjuntos cuja união é $K_{p}$, contradizendo que $K_{p}$ é conexo.

Provaremos agora a parte $(B)$. Sejam $\left(f_{n}\right)_{n \in \mathbb{N}},\left(\mu_{n}\right)_{n \in \mathbb{N}},\left(x_{n}\right)_{n \in \mathbb{N}}$ e $\varepsilon$ como nos itens de $(a)$ a $(d)$ da parte $(B)$.

Pelo teorema de Tietze existem funções contínuas $\tilde{f}_{n}:[0,1]^{I_{G}} \longrightarrow[0,1]$ tais que $\left.\tilde{f}_{n}\right|_{K}=f_{n}$. Por um teorema de Mibu (veja [Mi]) existe $I \subseteq I_{G}$ enumerável tal que, se $x|I=y| I$, então $\tilde{f}_{n}(x)=\tilde{f}_{n}(y)$, para todo $n$. 
Pela regularidade das medidas, cada $\mu_{n}$ depende de uma quantidade enumerável de abertos básicos. Logo, existem $J \subseteq I_{G}$ enumerável e medidas $\mu_{n}^{\prime}$ sobre $\pi_{J}[K]$ tais que para todo boreliano $A$ em $K$ temos $\mu_{n}(A)=\mu_{n}^{\prime}\left(\pi_{J}[A]\right)$. Podemos assumir que $J=I$.

Sejam $\dot{f}_{n}, \dot{\mu}_{n}^{\prime}, \dot{x}_{n}$ e $\dot{I} \mathbb{P}$-nomes para os objetos descritos acima. Pelo princípio do máximo (veja $[\mathrm{Ku}]$ ), podemos supor que $\mathbb{P}$ força que os itens de (a) a $(d)$ valem e que $\dot{I}$ satisfaz a tese do teorema de Mibu.

Para concluirmos $(B)$ precisamos provar que para todo $p \in \mathbb{P}$ existe $q \leq p$ que força os itens de $(e)$ a $(h)$.

Fixe $p \in \mathbb{P}$. Pelo lema 4.8 existe $r \leq p$ tal que $r \Vdash \dot{I} \subseteq \check{I}_{r}$. Por Mibu e por $\pi_{I_{r}}[K]=K_{r}$, achamos $\mathbb{P}$-nomes $\dot{g}_{n}$ para funções contínuas de $K_{r}$ em $[0,1]$ tais que

$$
r \Vdash \dot{g}_{n}\left(\dot{x} \mid \check{I}_{r}\right)=\dot{f}_{n}(\dot{x}), \text { para todo } \dot{\mathrm{x}} \in \dot{\mathrm{K}} \text {. }
$$

Claramente $r$ força que $\left(\dot{g}_{n}\right)_{n \in \mathbb{N}}$ são duas a duas disjuntas. Temos também que $r \Vdash \dot{J} \supseteq \check{I}_{r} \mathrm{e}$

$$
r \Vdash \dot{f}_{n} \circ \pi_{j}=\dot{g}_{n} \circ \pi_{j, \check{I}_{r}}
$$

para todo $n \in \mathbb{N}$.

Tomando $g_{n}=\operatorname{val}_{G}\left(\dot{g}_{n}\right)$, pelo lema 4.7 temos $g_{n} \in V$.

Repetindo o mesmo argumento e usando as medidas $\mu_{n}^{\prime}$, podemos assumir que existe uma sequência $\left(\nu_{n}\right)_{n \in \mathbb{N}}$ de medidas sobre $K_{r}$ tais que $\mu_{n}(A)=$ $\nu_{n}\left(\pi_{I_{r}}[A]\right)$, para todo boreliano $A$ em $K$.

Podemos também assumir que existem $z_{n} \in K_{r}$, para $n \in \mathbb{N}$, tais que

$$
r \Vdash \check{z}_{n}=\left.\dot{x}_{n}\right|_{\check{I}_{r}}
$$

Pelo lema 4.2 existem $q \leq r, \delta>0, b \subset a \subset \mathbb{N}$ subconjuntos infinitos, $z^{\prime} \in K_{q}$ e funções contínuas $g_{n}^{\prime}: K_{r} \longrightarrow[0,1]$ tais que $\operatorname{supp}\left(g_{n}^{\prime}\right) \subset \operatorname{supp}\left(g_{n}\right)$ e

(i) $\left|\int g_{n}^{\prime} d \nu_{n}\right|>\delta$ e $\Sigma\left\{\int g_{m}^{\prime} d\left|\nu_{n}\right|: m \neq n, m \in a\right\}<\delta / 3$, para todo $n \in a$;

(ii) $K_{q}=K_{r}\left(\left(g_{n}^{\prime}\right)_{n \in b}\right)$ é uma extensão completa;

(iii) $\left(g_{n}^{\prime} \circ \pi_{I_{q}, I_{r}}\right)_{n \in b}$ tem supremo em $C\left(K_{q}\right)$; 
(iv) $\left(\left(\pi_{I_{q}, I_{r}}^{-1}\left[\left\{z_{n}\right\}\right]\right)_{n \in \mathbb{N}}, b, a \backslash b, z^{\prime}\right) \in \mathcal{P}_{q}$.

Seja $\dot{g}$ um $\mathbb{P}$-nome para o supremo de $\left(\check{g}_{n}^{\prime} \circ \pi_{I_{q}, I_{r}}\right)_{n \in b}$ em $C\left(K_{q}\right)$. Podemos assumir que tal afirmação é forçada por $q$.

Em $V[G]$, defina $f_{n}^{\prime}=g_{n}^{\prime} \circ \pi_{I_{q}}$. Claramente vemos que vale $(e)$. O item $(f)$, por sua vez, segue de $(i)$. Para provar os itens $(g)$ e $(h)$ e concluir a demonstração do teorema é suficiente mostrar que

(v) $q \Vdash \dot{g} \circ \pi_{I_{q}}$ é o supremo de $\left(\dot{f}_{n}^{\prime}\right)_{n \in b}$ em $C(\dot{K}) \mathrm{e}$ $\overline{\left\{\dot{x}_{n}: n \in \check{b}\right\}} \cap \overline{\left\{\dot{x}_{n}: n \in \check{a} \backslash \check{b}\right\}} \neq \emptyset$.

Suponha que $q \Downarrow \dot{g} \circ \pi_{I_{q}}=\sup \left(\dot{f}_{n}^{\prime}\right)_{n \in b}$. Como

$$
q \Vdash \dot{f}_{n}^{\prime}(x)=\dot{g}_{n}^{\prime}\left(\left.x\right|_{\check{I}_{r}}\right)=\dot{g}_{n}^{\prime} \circ \pi_{I_{p}, I_{r}}(x) \leq \dot{g}\left(\left.x\right|_{\check{I}_{q}}\right)=\dot{g} \circ \pi_{I_{q}}(x)
$$

existem $s \leq q$ e um $\mathbb{P}$-nome $\dot{h}$ para uma função contínua de $K$ em $[0,1]$ tais que $s \Vdash \dot{f}_{n}^{\prime} \leq \dot{h}<\dot{g} \circ \pi_{\check{I}_{q}}$, para todo $n \in b$. Pelo teorema de Mibu existem $s^{\prime} \leq s$ e $\dot{J}$ tais que

$$
s^{\prime} \Vdash \dot{J} \subseteq \dot{I}_{G},|\dot{J}|=\check{\mathbb{N}} \text { e, se }\left.x\right|_{j}=\left.y\right|_{j} \text { então } \dot{h}(x)=\dot{h}(y)
$$

Pelo lema 4.8 existe $t \leq s^{\prime}$ tal que $t \Vdash \dot{J} \subseteq \check{I}_{t}$. Logo, existe $\mathbb{P}$-nome $\dot{h}^{\prime}$ para uma função contínua de $K_{t}$ em $[0,1]$ tal que

$$
t \Vdash \dot{h}=\dot{h}^{\prime} \circ \pi_{\check{I}_{t}}, \dot{g}_{n}^{\prime} \circ \pi_{\check{I}_{r}} \leq \dot{h}
$$

Como $t \leq s$, existe um $\mathbb{P}$-nome $\dot{x}$ para um elemento de $K$ tal que

$$
t \Vdash \dot{h}^{\prime}\left(\left.\dot{x}\right|_{\check{I}_{t}}\right)=\dot{h}(\dot{x})<\dot{g} \circ \pi_{\check{I}_{r}}(\dot{x})=\dot{g} \circ \pi_{\check{I}_{t}, \check{I}_{q}}\left(\left.\dot{x}\right|_{\check{I}_{q}}\right)
$$

Portanto, $t$ força que $\dot{g} \circ \pi_{\breve{I}_{t}, \breve{I}_{q}}$ não é o supremo de $\left(\dot{g}_{n}^{\prime} \circ \pi_{\check{I}_{r}}\right)_{n \in b}$ em $K_{r}$, pois

$$
t \Vdash \dot{g}_{n}^{\prime} \circ \pi_{\check{I}_{t}, \breve{I}_{r}} \leq \dot{h}^{\prime}<\dot{g} \circ \pi_{\check{I}_{t}, \breve{I}_{r}}
$$

Isso contradiz o lema 4.4. 
Para provarmos a segunda parte de $(v)$, suponha que

$$
q \Downarrow \overline{\left\{\dot{x}_{n}: n \in \check{b}\right\}} \cap \overline{\left\{\dot{x}_{n}: n \in \check{a} \backslash \check{b}\right\}} \neq \emptyset .
$$

Usando a compacidade de $K$, existem $s \leq q$ e $\mathbb{P}$-nomes $\dot{V}_{1}$ e $\dot{V}_{2}$ de abertos básicos de $K$ tais que

$$
s \Vdash \dot{V}_{1} \cap \dot{V}_{2}=\emptyset,\left\{\dot{x}_{n}: n \in \check{b}\right\} \subseteq \dot{V}_{1},\left\{\dot{x}_{n}: n \in \check{a} \backslash \check{b}\right\} \subseteq \dot{V}_{2}
$$

Seja $\dot{I}$ um $\mathbb{P}$-nome tal que $s \Vdash \dot{I}=\left\{\alpha \in \check{I_{G}}: \pi_{\{\alpha\}}\left[\dot{V}_{1}\right] \neq \pi_{\{\alpha\}}[\dot{K}]\right.$ ou $\left.\pi_{\{\alpha\}}\left[\dot{V}_{2}\right] \neq \pi_{\{\alpha\}}[\dot{K}]\right\}$.

Como $\dot{V}_{1}$ e $\dot{V}_{2}$ são $\mathbb{P}$-nomes para abertos básicos, podemos assumir que $s \Vdash|\dot{I}|<\omega$. Pelo lema 4.8 existe $t \leq s$ tal que

$$
t \Vdash \dot{I} \subseteq \check{I}_{t}
$$

Logo $t$ força que $\pi_{\check{I}_{t}}\left[\dot{V}_{1}\right]$ e $\pi_{\check{I}_{t}}\left[\dot{V}_{2}\right] \operatorname{separam}\left\{\left.\dot{x}_{n}\right|_{\check{I}_{t}}: n \in \check{b}\right\}$ e $\left\{\left.\dot{x}_{n}\right|_{\check{I}_{t}}: n \in \check{a} \backslash \breve{b}\right\}$ em $K_{t}$. I.e.,

$$
t \Vdash \overline{\left\{\left.\dot{x}_{n}\right|_{\check{I}_{t}}: n \in \check{b}\right\}} \cap \overline{\left\{\left.\dot{x}_{n}\right|_{\check{I}_{t}}: n \in \check{a} \backslash \check{b}\right\}}=\emptyset \mathrm{em} \check{\mathrm{K}}_{\mathrm{t}}
$$

Isso contradiz (iv) e as condições P3 e O4 da definição do forcing. De fato, por $(*)$ temos $\left.x_{n}\right|_{I_{t}} \in \pi_{I_{t}, I_{r}}^{-1}\left[\left\{z_{n}\right\}\right]$, para todo $n \in \mathbb{N}$. Além disso, por $(i v)$ e O4 existem $z^{\prime \prime} \in K_{t}$ e $a^{\prime}, b^{\prime} \subseteq \mathbb{N}$ subconjuntos infinitos e disjuntos tais que $\left(\left(\pi_{I_{t}, I_{r}}^{-1}\left[\left\{z_{n}\right\}\right]\right)_{n \in \mathbb{N}}, a^{\prime}, b^{\prime}, z^{\prime \prime}\right) \in \mathcal{P}_{t}$. Por P3 isso implicaria que $\left(\pi_{I_{t}, I_{r}}^{-1}\left[\left\{z_{n}\right\}\right]\right)_{n \in a^{\prime}}$ e $\left(\pi_{I_{t}, I_{r}}^{-1}\left[\left\{z_{n}\right\}\right]\right)_{n \in b^{\prime}}$ convergem para $z^{\prime \prime}$ e, como $a^{\prime} \backslash b$ e $b^{\prime} \backslash(a \backslash b)$ são finitos, obtemos uma contradição com $(* *)$.

A demonstração do item $(C)$ é a primeira parte da demonstração do Teorema 5.3 de [Fa2].

Por fim, para provarmos $(D)$ suponha primeiramente que $\mathbb{P}$ preserva cardinais. Suponha então que $K$ tem peso $\lambda<\kappa$ e seja $\mathcal{B}$ uma base para $K$ de cardinalidade $\lambda$. Sem perda de generalidade podemos assumir que cada elemento de $\mathcal{B}$ depende de um número finito de coordenadas e, portanto, pela regularidade de $\kappa$, existe $\alpha<\kappa$ tal que todo elemento de $\mathcal{B}$ depende das 
coordenadas abaixo de $\alpha$. Pelo lema 4.3 temos que

$$
D_{\alpha}=\left\{p \in \mathbb{P}: \exists \beta>\alpha\left(\beta \in I_{p} \wedge x_{\beta}^{p} \neq y_{\beta}^{p}\right)\right\}
$$

é denso em $\mathbb{P}$ e portanto $G \cap D_{\alpha} \neq \emptyset$. Seja $\tilde{p} \in G \cap D_{\alpha}$ e fixe $\beta>\alpha$ tal que $\beta \in I_{\tilde{p}} \wedge x_{\beta}^{\tilde{p}} \neq y_{\beta}^{\tilde{p}}$. Defina $x_{\beta}=\bigcup_{p \in G} x_{\beta}^{p}$ e $y_{\beta}=\bigcup_{p \in G} y_{\beta}^{p}$. Portanto, $x_{\beta} \neq y_{\beta}$ e existem elementos de $\mathcal{B}$ que separam $x_{\beta}$ e $y_{\beta}$. Por hipótese, a separação acontece abaixo de $\alpha$, o que é uma contradição pois por $\mathrm{P} 4$ e $\mathrm{O} 5$ temos que $x_{\beta}\left|\alpha=y_{\beta}\right| \alpha$. Isto encerra a demonstração do Teorema.

O espaço $K$ construído anteriormente tem peso pelo menos contínuo, e portanto $C(K)$ tem densidade pelo menos contínuo, pois o peso de $K$ é a densidade de $C(K)$. De fato, $K$ tem peso pelo menos contínuo porque todo espaço fracamente Koszmider tem peso não-enumerável (veja [Ko2]). Como começamos em um modelo $V$ onde vale $\mathrm{CH}$ e usamos um forcing $\sigma$-fechado (que não adiciona conjuntos enumeráveis), CH continua valendo na extensão. Não obstante, pelo item $(D)$ do teorema 4.9 e pelo lema 4.6 , temos que se $\mathbb{P}$ for $\omega_{2}$-c.c então $K$ terá peso $\kappa$ estritamente maior que contínuo, e portanto $C(K)$ terá densidade maior que contínuo, respondendo positivamente a seguinte pergunta:

Pergunta 1. É relativamente consistente com ZFC que existe um espaço topológico compacto, Hausdorff e conexo $K$ tal que $C(K)$ tem densidade maior que contínuo e $K$ é hereditariamente fracamente Koszmider?

Para o forcing que construímos, não sabemos se na extensão $V[G]$ vale o princípio $\diamond$. Se em $V[G]$ valer $\neg \diamond, K$ responderá positivamente a seguinte pergunta:

Pergunta 2. É relativamente consistente com $C H+\neg \diamond$ que existe um espaço topológico compacto, Hausdorff e conexo $K$ hereditariamente fracamente Koszmider?

A Pergunta 2 é importante pois a única construção que conhecemos de $K$ hereditariamente fracamente Koszmider é feita assumindo $\diamond$. Veja [Fa2] para maiores referências. 


\subsection{Um resultado sobre a densidade de $C(K)$ com poucos operadores}

Em [Ko4], Koszmider mostra que uma condição suficiente sobre $K$ para garantir que $C(K)$ tenha poucos operadores é a seguinte: existe $E \subseteq K$ denso tal que para toda sequência $\left(x_{n}\right)_{n \in \mathbb{N}}$ em $E$ e toda sequência de abertos $\left(U_{n}\right)_{n \in \mathbb{N}} \subseteq K$ tais que $x_{n} \notin U_{m}$ para todos $m$ e $n$, existe $M \subseteq \mathbb{N}$ infinito e co-infinito satisfazendo $\overline{\left\{x_{n}: n \in M\right\}} \cap \overline{\left\{x_{n}: n \in \mathbb{N} \backslash M\right\}} \neq \emptyset \mathrm{e}$ $\overline{\bigcup\left\{U_{n}: n \in M\right\}} \cap \overline{\bigcup\left\{U_{n}: n \in \mathbb{N} \backslash M\right\}}=\emptyset$. Mostraremos agora que essa condição, comumente utilizada nas construções de espaços de Banach $C(K)$ com poucos operadores, impõe um limitante superior para a densidade de $C(K)$, ou equivalentemente, para o peso de $K$.

Lema 4.10. Se $K$ é um espaço topológico compacto de densidade $\kappa$ e $E \subseteq K$ é um denso, então existe uma sequência $\left(X_{\alpha}\right)_{\alpha \in \kappa}$ de subconjuntos de E tais que cada $X_{\alpha}=\left\{x_{n}^{\alpha}: n \in \mathbb{N}\right\}$, onde $\left(x_{n}^{\alpha}\right)_{n \in \mathbb{N}}$ é uma sequência infinita e relativamente discreta, e $\overline{X_{\beta}} \cap X_{\alpha}=\emptyset \forall \beta<\alpha$.

Demonstração. Construíremos a sequência $\left(X_{\alpha}\right)_{\alpha \in \kappa}$ por recursão transfinita. Para isso, fixemos $\alpha<\kappa$ e assumamos que já tenhamos construído $X_{\beta} \subseteq E$, para $\beta<\alpha$. Defina agora $X=\bigcup_{\beta<\alpha} X_{\beta}=\bigcup_{\beta<\alpha}\left\{x_{n}^{\beta}: n \in \mathbb{N}\right\}$. Como $|X|<d(K)=\kappa$, temos que $\bar{X} \neq K$ e, mais ainda, que $K \backslash \bar{X}$ é um aberto infinito. Como $E$ é denso, segue que $(K \backslash \bar{X}) \cap E$ é infinito. Daí, podemos tomar $\left(x_{n}^{\alpha}\right)_{n \in \mathbb{N}}$ uma sequência infinita e relativamente discreta em $E \backslash \bar{X}$ e definir $X_{\alpha}=\left\{x_{n}^{\alpha}: n \in \mathbb{N}\right\}$. Para ver que a sequência $\left(X_{\alpha}\right)_{\alpha \in \kappa}$ satisfaz a condição do enunciado, tome $\beta<\alpha$ quaisquer. Como $X_{\alpha} \subseteq E \backslash \overline{X_{\beta}} \subseteq K \backslash \overline{X_{\beta}}$, segue que $\overline{X_{\beta}} \cap X_{\alpha}=\emptyset$. Isto encerra a prova do lema.

Lema 4.11. ([Ju], 2.7) Seja $K$ um espaço topológico compacto ${ }^{1}$. Então $w(K) \leq 2^{d(K)}$.

Teorema 4.12. Seja K um espaço topológico compacto e suponha que existe $E \subseteq K$ denso tal que para toda sequência $\left(x_{n}\right)_{n \in \mathbb{N}}$ em $E$ e toda sequência

\footnotetext{
${ }^{1} \mathrm{O}$ mesmo resultado vale para espaços topológicos regulares em geral.
} 
de abertos $\left(U_{n}\right)_{n \in \mathbb{N}} \subseteq K$ tais que $x_{n} \notin U_{m}$ para todos $m$ e $n$, existe $M \subseteq \mathbb{N}$ infinito e co-infinito satisfazendo $\overline{\left\{x_{n}: n \in M\right\}} \cap \overline{\left\{x_{n}: n \in \mathbb{N} \backslash M\right\}} \neq \emptyset e$ $\overline{\bigcup\left\{U_{n}: n \in M\right\}} \cap \overline{\bigcup\left\{U_{n}: n \in \mathbb{N} \backslash M\right\}}=\emptyset$. Então $w(K) \leq 2^{2^{2^{\omega}}}$.

Demonstração. Sejam $d(K)=\kappa$ e $E \subseteq K$ denso. Tome $\left(x_{n}^{\alpha}\right)_{n \in \mathbb{N}, \alpha \in \kappa}$ como no Lema 4.10 e considere a aplicação $\varphi: \kappa \longrightarrow \mathcal{P}(\mathcal{P}(\mathbb{N}))$ dada por $\varphi(\alpha)=$ $\left\{M \subseteq \mathbb{N}\right.$ infinito e co-infinito : $\left.\overline{\left\{x_{n}^{\alpha}: n \in M\right\}} \cap \overline{\left\{x_{n}^{\alpha}: n \in \mathbb{N} \backslash M\right\}} \neq \emptyset\right\}$. Por absurdo, suponha que $\kappa>2^{2^{\omega}}$. Então existem $\alpha, \beta \in \kappa$ tais que $\beta<\alpha$ e $\varphi(\beta)=\varphi(\alpha)$. Usando a normalidade de $K$ e o fato de que $\overline{\left\{x_{n}^{\beta}: n \in \mathbb{N}\right\}} \cap\left\{x_{n}^{\alpha}: n \in \mathbb{N}\right\}=\emptyset$, podemos tomar $\left(U_{n}\right)_{n \in \mathbb{N}} \subseteq K$ uma sequência de abertos não vazios dois a dois disjuntos satisfazendo $x_{n}^{\alpha} \in U_{n}$ e $x_{n}^{\beta} \notin U_{m}$ para todos $m$ e $n$. Por hipótese, existe $M \subseteq \mathbb{N}$ infinito e coinfinito tal que $\overline{\left\{x_{n}^{\beta}: n \in M\right\}} \cap \overline{\left\{x_{n}^{\beta}: n \in \mathbb{N} \backslash M\right\}} \neq \emptyset$ e $\overline{\bigcup\left\{U_{n}: n \in M\right\}} \cap$ $\overline{\bigcup\left\{U_{n}: n \in \mathbb{N} \backslash M\right\}}=\emptyset$. Em particular, $M \in \varphi(\beta)$ e portanto $M \in \varphi(\alpha)$. Dessa forma, segue que devemos ter $\overline{\left\{x_{n}^{\alpha}: n \in M\right\}} \cap \overline{\left\{x_{n}^{\alpha}: n \in \mathbb{N} \backslash M\right\}} \neq \emptyset$, o que é um absurdo pois sabemos que $\overline{\left\{x_{n}^{\alpha}: n \in M\right\}} \cap \overline{\left\{x_{n}^{\alpha}: n \in \mathbb{N} \backslash M\right\}} \subseteq$ $\overline{\bigcup\left\{U_{n}: n \in M\right\}} \cap \overline{\bigcup\left\{U_{n}: n \in \mathbb{N} \backslash M\right\}}=\emptyset$. Desse modo, concluímos que $\kappa \leq 2^{2^{\omega}}$ e portanto, pelo Lema 4.11 , temos $\omega(K) \leq 2^{d(K)} \leq 2^{2^{2^{\omega}}}$.

Em [Ko6] os autores provam que não existe limitante superior para a densidade dos espaços de Banach da forma $C(K)$ com poucos operadores. A técnica utilizada é diferente das técnicas usuais e ela se baseia nas ideias contidas em [Sh1] e [Sh2]. Motivados pela Pergunta 1 da seção anterior e pelo resultado de [Ko6], é razoável formularmos a seguinte pergunta:

Pergunta 3. Será que existe limitante superior para a densidade dos espaços de Banach da forma $C(K)$ que satisfazem a propriedade de que $C(L)$ tem poucos operadores, para todo fechado $L \subseteq K$ ? 


\section{Bibliografia}

[AG] Aiena, P.; Gonzalez, M. Essentially incomparable Banach spaces and Fredholm Theory. Proc. Irish. Acad. Sect. A93, n. 1, p. 49 - 59, 1993.

[BF] Barbeiro, A.S.V.; Fajardo, R.A.S. Suprema of continuous functions on connected spaces. São Paulo J. Math. Sci, 2016.

[Ci] Ciesielski, K. Set theory for the working mathematician. Cambridge University Press, Cambridge, 1997.

[Di] Diestel, J. Sequences and series in Banach spaces. Spring-Verlag, New York, 1984.

[DU] Diestel, J.; Uhl, J. J. Jr. Vector measures. Mathematical Surveys 15, AMS. 1977.

[Do] Douwen, E. K. The integers and topology. Em Kunen, K.; Vaughan, J. E., editores, Handbook of set-theoretic topology. Elsevier Science Publishers B.V., p. 111 - 167, 1984.

[DS] Dunford, N.; Schwartz, J. T. Linear operators. Interscience Publishers LTD., London, 1958.

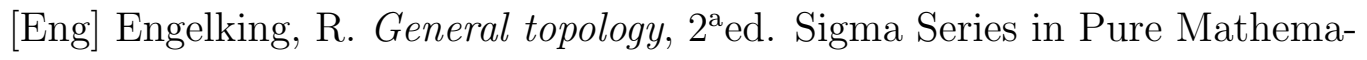
tics, 6, Heldermann Verlag, Berlin, 1989.

[Fa] Fabian, M.; Hájek, P.; Pelant, J.; Habala, P.; Montesinos, V.; Zizler, V. Functional analysis and infinite-dimensional geometry. Spring-Verlag, New York, 2001.

[Fa1] Fajardo, R. An indecomposable Banach space of continuous functions which has small density. Fund. Math., v. 202, n. 1, p. 43 - 63, 2009.

[Fa2] Fajardo, R. Quotients of indecomposable Banach spaces of continuous 
functions. Studia Math., v. 212, n. 3, p. 259 - 283, 2012.

[Fa3] Fajardo, R. Essentially Incomparable Banach Spaces of Continuous Functions. Bull. Pol. Ac.: Tech., v. 58, n. 3, p. 247 - 258, 2010.

[Fed] Fedorchuk, V.V. A bicompactum whose infinite closed subsets are all n-dimensinal (Russo). Math. USSR Sbornik, v. 25, p. 37 - 57, 1975.

[Fer] Ferenczi, V. Quotient hereditarily indecomposable Banach spaces. Canad. J. Math., v. 51, n. 3, p. 566 - 584, 1999.

[Fr] Fremlin, D. H. Consequences of Martin's axiom. Cambridge University Press, Cambridge, 1984.

[Ga] Gasparis, I. A continuum of totally incomparable hereditarily indecomposable Banach spaces. Studia Math., v. 151, n. 3, p. 277 - 298, 2002.

[Go] Gödel, K. What is Cantor's continuum problem? Amer. Math. Monthly., v.54, p. $515-525,1947$.

[GM1] Gowers, W. T; Maurey, B. The unconditional basic sequence problem. Journal A. M. S., v. 6, p. 851 - 874, 1993.

[GM2] Gowers, W. T; Maurey, B. Banach spaces with small spaces of operators. Math. Ann., v. 307, p. 543 - 568, 1997.

[Ha] Haydon, R.A non-reflexive Grothendieck space that does not contain $l_{\infty}$. Israel J. Math., v. 40, n. 1, p.65 - 73, 1981.

[HLO] Haydon, R.; Levy, M.; Odell, E. On sequences without weak* convergent convex block subsequences. Proc. Amer. Math. Soc., v.100, n. 1, p. 94 - 98, 1987.

[Ju] Juhász, I. Cardinal functions in topology - ten years later. Math. Centre Tracts, Vol. 123, Math. Centrum, Amsterdam, 1980.

[Je] Jech, T. Set Theory : The Third Millennium Edition, revised and expanded. Springer-Verlag, Berlin, 2002.

[KP] Kadec, M. I.; Pełczyński, A. Bases, lacunary sequences and complemented subspaces in the space $L_{p}$. Studia Math., v. 21, p. 161 - 176, 1962.

[Ko1] Koszmider, P. Forcing minimal extensions of Boolean algebras. Trans. Amer. Math. Soc., v. 351, n. 8, p. 3073 - 3117, 1999. 
[Ko2] Koszmider, P. Banach spaces of continuous functions with few operators. Math. Annalen., v. 330, p. 151 - 183, 2004.

[Ko3] Koszmider, P. A space $C(K)$ where all nontrivial complemented subspaces have big densities. Studia Math., v. 168, n. 2, p. 109 - 127, 2005.

[Ko4] Koszmider, P. A survey on Banach spaces $C(K)$ with few operators. Rev. R. Acad. Cienc. Exactas Fis. Nat. Ser. A Math. RACSAM 104 (2010), no. 2, 309-326.

[Ko5] Koszmider, P. On large indecomposable Banach spaces. J. Funct. Anal., v. 264, p. 1779 - 1805, 2013.

[Ko6] Śwetek, M.; Koszmider, P.; Shelah, S. There is no bound on sizes of indecomposable Banach spaces. Advances in Mathematics, v. 323, p. 745 $783,2018$.

[Kop] Koppelberg, S. General theory of Boolean algebras. Em Monk, J.D., editor, Handbook of Boolean Algebras. Elsevier Science Publishers B.V., Amsterdam, 1989.

[Ku] Kunen, K. Set Theory. An Introduction to Independence Proofs. North Holland, 1980.

[LM] Lacey, E.; Morris, P. On spaces of the type $A(K)$ and their duals. Proc. Amer. Math. Soc., v. 23, p. 151 - 157, 1969.

[LT] Lindenstrauss, J.; Tzafriri, L. Classical Banach Spaces I: Sequences Spaces. Springer-Verlag, 1977.

[Me] Meyer-Nieberg, P. Banach Lattices. Universitext, Springer-Verlag, Berlin, 1991.

[Mi] Mibu; On Baire functions on finite product spaces. Proc. Imperial Acad. Tokyo, v. 20, p. 661 - 663, 1994.

[Pe1] Pełczyński, A. Banach spaces on which every unconditionally converging operation is weakly compact. Bull. Acad. Polon. Sc. Ser. Sci. Math. Astronom. Phys., v. 10, p. 641 - 648, 1962.

[Pe2] Pełczyński, A. On strictly singular and strictly non-singular operators I. Bull. Acad. Polon. Sc. Ser. Sci. Math. Astronom. Phys., v. 13, p. 31 41, 1962. 
[Pl] Plebanek, G.; A construction of a Banach space $C(K)$ with few operators. Top. Appl., v. 143, p. 217 - 239, 2004.

[Ru] Rudin, W. Real and Complex Analysis, $3^{\mathrm{a}}$ ed. McGraw-Hill, New York, 1987.

[Sc] Schachermayer, W. On some classical measure-theoretic theorems for non-sigma-complete Boolean algebras. Diss. Math., 214, 1982.

[Schl] Schlackow, I. Centripetal operators and Koszmider spaces. Top. Appl., v. 155, p. 1227 - 1236, 2008.

[Se] Semadeni, Z.Banach spaces of continuous functions. Państwowe Wydawnictwo Naukowe, 1971.

[Sh1] Shelah, S. Existence of endo-rigid Boolean algebras. Lecture Notes in Math., v. 1182, p. 91 - 119, 1986.

[Sh2] Shelah, S. Existence of endo-rigid Boolean algebras. preprint, https://arxiv.org/abs/1105.3777, 2011.

[SS] Shelah, S.; Steprans, J.; A Banach Space on which there are few operators. Proc. Amer. Math. Soc., v. 104, p. 101 - 105, 1988.

[Ta] Talagrand, M. Un Nouveau $C(K)$ qui possede la propriete de Grothendieck. Israel J. Math., v. 37, p. 181 - 191, 1980.

[Ve] Velleman, D. Morasses, diamond and forcing. Ann. Pure. Appl. Logic, v. 23, p. $199-281,1983$.

[Wa] Walker, R. The Stone-Čech Compactification. Springer-Verlag, Berlin, 1974. 